\title{
Metalevel Argumentation
}

\author{
Sanjay Modgil, Trevor Bench-Capon ${ }^{\dagger}$
}

\begin{abstract}
The abstract nature of Dung's seminal theory of argumentation accounts for its widespread application as a general framework for various species of nonmonotonic reasoning, and, more generally, reasoning in the presence of conflict, whether such conflict arises given uncertain or incomplete information or as a result of differing opinions or preferences. In this paper we formalise reasoning about argumentation within the Dung argumentation paradigm itself. A metalevel Dung argumentation framework is itself instantiated by arguments that make statements about arguments, their interactions, and their evaluation in an object-level argumentation framework. We show how Dung's theory, and object level extensions of Dung's theory, such as those intended to accommodate preferences, can then be uniformly characterised by metalevel argumentation in a Dung framework. We therefore formalise a range of extensions to Dung's theory, within the Dung paradigm itself, and show how this then provides for application of the full range of theoretical and practical developments of Dung's theory, to extensions of Dung's theory, and combination and further augmentation of these extensions. Furthermore, in the spirit of Dung's original theory, metalevel frameworks adopt a level of abstraction that makes limited commitments to the instantiating logics. Metalevel frameworks thus provide principled means for instantiation by, and integration of arguments constructed from different underlying logics, where one logic may encode metalevel reasoning about the arguments and attacks defined by a theory in another logic.
\end{abstract}

Keywords: Argumentation, Dung, Metalevel, Preferences, Values, Non-monotonic reasoning, Conflict Resolution

\section{Introduction}

\subsection{Background}

The formal study of argumentation has come to be a core study within Artificial Intelligence [14]. Logic based models of argumentation have been applied to formalisation of conflict resolution in propositional and first order classical logics [2, 17], non-monotonic logics [21, 27, 31], to defeasible reasoning and conflict resolution over the full gamut of agents' mental attitudes [1, 37], and to decision making over actions $[7,34]$. The inherently dialectical nature of these models have been exploited in the development of argument game proof theories for argumentation [24, 28, 42, 53], and foundations for formalisation of argumentation-based dialogues [4, 32, 47]. Furthermore, recent major research projects $[5,6,30]$ have developed general models of

\footnotetext{
*Corresponding author: Sanjay Modgil (sanjaymodgil@yahoo.co.uk), Department of Computer Science, Imperial College London, Queen's Gate, London SW7 2AZ, UK (+44 (0)788 307 5206)

${ }_{\dagger}^{\dagger}$ T.J.M Bench-Capon (tbc@ csc.liv.ac.uk) Department of Computer Science, University of Liverpool, Liverpool L69 7ZF, UK
} 
argumentation based inference, decision making and dialogue, and implementations of these models for deployment in agent and semantic grid applications.

Many of the above theoretical and practical developments build on Dung's seminal theory of argumentation [27]. A Dung argumentation framework is a directed graph consisting of a set of arguments $\mathcal{A}$ and a binary conflict based attack relation $\mathcal{R}$ on $\mathcal{A}$. The extensions, and so justified arguments of a framework are then defined under different semantics, where the choice of semantics equates with varying degrees of scepticism or credulity. Extensions are defined through application of an 'acceptability calculus', whereby an argument $x \in \mathcal{A}$ is said to be acceptable with respect to $S \subseteq \mathcal{A}$, iff any argument $y$ that attacks $x$ is itself attacked by some argument $z$ in $S$. For example, if $S$ is a maximal (under set inclusion) set such that all its contained arguments are acceptable with respect to $S$, then $S$ is said to be an extension under the preferred semantics.

Dung's theory has been developed in a number of directions. Some works have motivated and formalised collective attacks between sets of, rather than single, arguments $[18,44]$. Other works formalise the role of preferences, so that an argument $x$ successfully attacks $y$ iff $y$ is not preferred to $x$ according to some given preference relation on $\mathcal{A}$ [2], or the value promoted by $y$ is not ranked higher than the value promoted by $x$, according to a given ordering on values [12]. More recently, [38, 40]'s Extended Argumentation Framework (EAF) extends Dung's framework to include arguments that attack attacks. Thus, if $x$ and $y$ attack each other, then an argument $z$ justifying a preference for $y$ over $x$, attacks the attack from $x$ to $y$. In this way, the extended argumentation framework allows for argumentation based reasoning about possibly conflicting preference information to be accommodated within the argumentation framework itself. In [8], this idea has been further generalised so that not only can arguments attack attacks, but these attacks on attacks can themselves be attacked. Finally, a number of works augment Dung's framework to include a support relation on arguments $[3,45]$.

The continuing development and widespread influence and application of Dung's ideas can be attributed to the abstract nature of a Dung argumentation framework, and to the encoding of intuitive generic principles of commonsense reasoning in the acceptability calculus, embodying the key insight that an argument is not acceptable or unacceptable in itself, but relative to the context of other available arguments, not only those which attack it, but also those which attack those attackers. Its abstract nature allows for instantiation by various logical formalisms; one is free to choose a logic $\mathcal{L}$ and define what constitutes an argument and attack between arguments defined by a theory in $\mathcal{L}$. Thus, a theory's inferences can then be defined in terms of the claims of the justified arguments constructed from the theory (an argument essentially being a proof of a candidate inference - the argument's claim - in the underlying logic). Indeed, many logic programming formalisms and non-monotonic logics (e.g. default, auto-epistemic, non-monotonic modal logics, certain instances of circumscription, and defeasible logic) have been shown to conform to Dung's semantics [21, 26, 27, 31], thus testifying to the general applicability of the principles encoded in the acceptability calculus. Dung's theory can therefore be understood as a semantics for non-monotonic reasoning. In this view, what appropriately accounts for the correctness of an inference is that an argument for the inference can be shown to rationally prevail in the face of arguments for opposing inferences, where, one can claim that: it is application of the acceptability calculus that encodes logic neutral, rational means for establishing such standards of correctness. 


\subsection{Overview of Paper}

In this paper we further substantiate the above claim, by formalising reasoning about argumentation within the Dung argumentation paradigm itself. The basic idea is that given an object-level argumentation framework, one can consider metalevel arguments that can be explicitly categorised according to the types of claim made about the arguments and their relations in the object level framework. These metalevel arguments can then themselves be related by an attack relation in a Dung framework, where this metalevel attack relation satisfies constraints imposed by the claim based categorisation. One can then show a correspondence between the object level framework and its metalevel formulation, such that the justified arguments of the object level framework can be computed directly from its metalevel formulation.

For example, given an object level Dung framework $(\mathcal{A}, \mathcal{R})$ one can consider metalevel arguments that make claims such as ' $x$ is justified', ' $x$ is rejected', ' $x$ attacks $y$ ', about arguments $x, y \in \mathcal{A}$. These metalevel arguments can themselves be organised into a Dung framework such that an argument claiming ' $x$ is justified' is a justified argument of the metalevel framework, iff $x$ is a justified argument of the object level framework. Thus, the acceptability calculus applied at the metalevel characterises the use of the acceptability calculus at the object level.

The remainder of this paper is organised as follows. Section 2 reviews Dung's abstract argumentation theory, and the various developments of the theory referred to above. In Section 3 we augment a Dung argumentation framework $(\mathcal{A}, \mathcal{R})$ to obtain a 5 tuple Structured Argumentation Framework $(S A F)$ that includes a function, a language and a set of constraints, such that the function maps arguments in $\mathcal{A}$ to claims in the language, and given this claim based categorisation of arguments, a set of constraints on $\mathcal{R}$ is specified. We then define a specific language in which one can express claims about object level frameworks, and so identify Metalevel Argumentation Frameworks ( $M A F \mathrm{~s}$ ) as a special class of $S A F \mathrm{~s}$. We then show how Dung frameworks, their generalisation to accommodate collective attacks, their extensions to accommodate preferences [2] and values [12], and a special, but widely applicable, class of [38]'s extended framework, can all be formulated as instances of metalevel argumentation.

In Section 4 we discuss some implications and applications of metalevel argumentation. Firstly, since $M A F$ s formalise Dung argumentation and many of its developments, within the Dung paradigm itself, one can transition the full range of theoretical results and techniques for Dung argumentaton, to developments of Dung argumentation. We illustrate by showing how standard argument game proof theories developed for Dung frameworks can now be applied to the metalevel formulation of value based argumentation [12], and contrast the use of such games with the more complex games specifically developed for value based argumentation [11,13]. Secondly, in the spirit of Dung's original theory, $M A F$ s adopt a level of abstraction that makes limited commitments to the instantiating logics. Thus metalevel arguments can be instantiated based on the existence of arguments in different object level frameworks, which in turn may be constructed from different underlying logics. This not only allows for integration and further extension of different forms of abstract argumentation, but also provides principled means for instantiation by, and integration of arguments constructed from different underlying logics, where one logic may encode metalevel reasoning about the arguments and attacks defined by a theory in another logic. Section 4 illustrates these applications by discussing how value based argumentation can be extended to accommodate argumentation over different rankings of values, and how one can integrate 
arguments expressing preferences and values, and how the arguments instantiating such an integration can be constructed from different underlying logical theories. In Section 5 we discuss related work, and conclude in Section 6 in which we also point to future work.

To summarise, the contributions of this paper are as follows ${ }^{1}$ :

1. We formalise abstract metalevel argumentation frameworks that adopt the same basic machinery of a Dung framework, but overlay more structure by identifying classes of claims about arguments in object level frameworks, and thus constraints on the attack relation.

2. Dung's abstract argumentation theory can be said to identify general dialectical principles that underpin common-sense reasoning as encoded in a range of nonmonotonic reasoning formalisms. This paper promotes and substantiates this view by showing how a number of developments of Dung argumentation can be uniformly characterised in terms of these dialectical principles.

3. We show how by formalising Dung argumentation and its developments, within the Dung paradigm itself, the full range of theoretical and practical results and techniques for Dung's work can be applied to its developments.

4. We show how metalevel argumentation frameworks provide a unifying formalism in which to integrate and further extend the various developments of Dung argumentation, and provide principled means for instantiation by, and integration of arguments constructed from different underlying logics and theories, where one theory may encode metalevel reasoning about the arguments defined by another theory in another logic.

\section{Abstract Argumentation Theories}

\subsection{Dung's Theory of Abstract Argumentation}

We review Dung's extension-based approach to evaluating the status of arguments [27], and then review the more recent labelling approach [22, 23, 51, 52]. We then review a recent generalisation of Dung's theory to accommodate collective attacks.

\subsubsection{Dung's Extension-based Argumentation Semantics}

Definition 1 [Dung Argumentation Framework] A Dung argumentation framework is a tuple $(\mathcal{A}, \mathcal{R})$, where $\mathcal{A}$ is a set of arguments, and $\mathcal{R} \subseteq(\mathcal{A} \times \mathcal{A})$ is a binary attack relation on $\mathcal{A}$.

Figure 1 shows a Dung argumentation framework $(A F)$ in which an arrow from $x$ to $y$ denotes that $x \mathcal{R} y$.

The notion of an argument being acceptable with respect to (w.r.t.) a set of arguments is then defined:

Definition 2 [Acceptability for Dung Frameworks] Let $(\mathcal{A}, \mathcal{R})$ be an $A F$, and $S \subseteq$ $\mathcal{A}$. Then $x \in \mathcal{A}$ is acceptable w.r.t. $S$ iff for all $y \in \mathcal{A}$ such that $y \mathcal{R} x$, there exists a $z \in S$ such that $z \mathcal{R} y$

\footnotetext{
${ }^{1}$ This paper builds on and substantially extends work first presented in [41]. This work is briefly reviewed in Section 5
} 


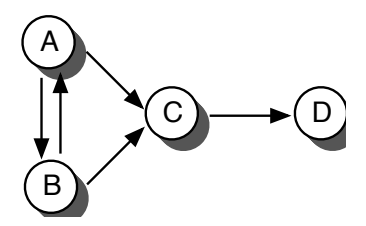

Figure 1: A Dung argumentation framework

The acceptability of arguments underpins evaluation of the status of arguments. If $S$ is conflict free (no arguments in $S$ attack each other), and all arguments in $S$ are acceptable w.r.t. $S$, then $S$ is said to be admissible. The status of arguments, under either a credulous or sceptical perspective, is then evaluated w.r.t. the extensions of a framework defined under different semantics. These semantics 'globalise' the essentially local notion of admissibility, by specifying properties that identify a subset of all the admissible extensions.

Definition 3 [Extensions of a Dung Framework] Let $\Delta=(\mathcal{A}, \mathcal{R})$. Let $S \subseteq \mathcal{A}$ such that $\forall x, y \in S$, it is not the case that $x \mathcal{R} y$, in which case $S$ is said to be conflict free. Then:

1. $S$ is an admissible extension of $\Delta$ iff each argument in $S$ is acceptable w.r.t. $S$

2. $S$ is a complete extension of $\Delta$ iff $S$ is admissible, and every argument in $\mathcal{A}$ that is acceptable w.r.t. $S$, is in $S$

3. $S$ is the grounded extension of $\Delta$ iff $S$ is the minimal (w.r.t. set inclusion) complete extension

4. $S$ is a preferred extension of $\Delta$ iff $S$ is a maximal (w.r.t. set inclusion) complete extension

5. $S$ is a stable extension of $\Delta$ iff $S$ is an admissible extension such that every argument not in $S$ is attacked by an argument in $S$

Definition 4 [Status of arguments in a Dung Framework] Let $\Delta=(\mathcal{A}, \mathcal{R})$. For $s \in\{$ complete, preferred, stable, grounded $\}$ :

- If $x \in \mathcal{A}$ is in at least one $s$ extension of $\Delta$ then $x$ is said to be credulously justified under the $s$ semantics.

- If $x \in \mathcal{A}$ is in all $s$ extensions of $\Delta$ then $x$ is said to be sceptically justified under the $s$ semantics.

- If $x \in \mathcal{A}$ is not in any $s$ extension of $\Delta$ then $x$ is said to be rejected under the $s$ semantics.

The admissible extensions of Figure 1's $A F$ are: $\emptyset,\{a\},\{b\},\{a, d\}$, and $\{b, d\}$. The complete extensions are $\emptyset,\{a, d\}$, and $\{b, d\}$. The preferred and stable extensions are $\{a, d\}$, and $\{b, d\}$, and $\emptyset$ is the grounded extension. Note that $d$ is sceptically justified under the preferred, but not under the grounded, semantics. All arguments are rejected under the grounded semantics, whereas only $c$ is rejected under the preferred and stable semantics.

We introduce some notation that will be of use in the remainder of this paper: 
Notation 1 Let $(\mathcal{A}, \mathcal{R})$ be an $A F$, and $E \subseteq \mathcal{A}$.

- $\overrightarrow{E+}$ denotes the set of attacks originating from arguments in $E$ :

$\overrightarrow{E+}=\{(x, y) \mid x \in E, x \mathcal{R} y\}$

- $E+$ denotes the set of arguments attacked by arguments in $E$ :

$E+=\{y \mid x \in E, x \mathcal{R} y\}$

- E- denotes the set of arguments that attack arguments in $E$ :

$E-=\{y \mid y \mathcal{R} x, x \in E\}$

\subsubsection{Labellings for Argumentation Frameworks}

The extensions and status of arguments in a Dung framework can be defined in terms of labellings [22, 23, 51,52]. Here, we review the labelling approach presented in $[22,23]$.

Definition 5 [Labelling function] A labelling is a total function $\mathcal{L}$ that assigns a label IN, OUT or UNDEC to each argument $x \in \mathcal{A}$ in an argumentation framework $(\mathcal{A}, \mathcal{R})$. Henceforth, we say that:

- $\operatorname{in}(\mathcal{L})=\{x \mid \mathcal{L}(x)=$ IN $\}$

- $\operatorname{out}(\mathcal{L})=\{x \mid \mathcal{L}(x)=$ OUT $\}$

- $\operatorname{undec}(\mathcal{L})=\{x \mid \mathcal{L}(x)=\operatorname{UNDEC}\}$

Legal labellings of arguments are then defined, and used as a basis for characterising the extensions of an $A F$.

Definition 6 [Legal labellings] Let $\mathcal{L}$ be a labelling for $(\mathcal{A}, \mathcal{R})$ and $x \in \mathcal{A}$.

- $x$ is legally IN iff $x$ is labelled IN and every $y$ that attacks $x$ is labelled OUT

- $x$ is legally OUT iff $x$ is labelled OUT and there is at least one $y$ that attacks $x$ and $y$ is labelled IN

- $x$ is legally UNDEC iff there is no $y$ that attacks $x$ such that $y$ is labelled IN, and it is not the case that: for all $y \in \mathcal{A}$ if $y$ attacks $x$, then $y$ is labelled OUT ${ }^{2}$

Definition 7 [Labellings for Extensions] For $1 \in\{$ IN, OUT, UNDEC $\}$ an argument $x$ is said to be illegally $l$ iff $x$ is labelled $l$, and it is not legally $l$.

- An admissible labelling $\mathcal{L}$ is a labelling without arguments that are illegally IN and without arguments that are illegally OUT.

- A complete labelling $\mathcal{L}$ is an admissible labelling without arguments that are illegally UNDEC

Let $\mathcal{L}$ be a complete labelling. Then:

- $\mathcal{L}$ is a grounded labelling iff there there does not exist a complete labelling $\mathcal{L}^{\prime}$ such that $\operatorname{in}\left(\mathcal{L}^{\prime}\right) \subset \operatorname{in}(\mathcal{L})$

\footnotetext{
${ }^{2}$ In other words, $x$ is legally UNDEC iff it has at least one attacker that is labelled UNDEC and no attacker that is labelled IN
} 
- $\mathcal{L}$ is a preferred labelling iff there there does not exist a complete labelling $\mathcal{L}^{\prime}$ such that $\operatorname{in}\left(\mathcal{L}^{\prime}\right) \supset \operatorname{in}(\mathcal{L})$

- $\mathcal{L}$ is a stable labelling iff undec $(\mathcal{L})=\emptyset$

In [23], the following theorem is shown to hold:

Theorem 1 Let $\Delta=(\mathcal{A}, \mathcal{R})$. For $s \in\{$ admissible, complete, grounded, preferred, stable $\}: E$ is an $s$ extension of $\Delta$ iff there exists an $s$ labelling $\mathcal{L}$ with $\operatorname{in}(\mathcal{L})=E$.

Notice the extra expressivity compared with the extension based approach. An $s$ labelling $\mathcal{L}$ not only identifies the arguments in an $s$ extension $E$ (the arguments labelled IN), but also the arguments in $E+$ and $E-$ (the union of which are the arguments labelled OUT). Note that since each $s$ extension is admissible, it then follows that it is always the case that $E-\subseteq E+$. Also note that those arguments labelled UNDEC are neither in $E, E+$ or $E-$, and so are the arguments that are neither in the $s$ extension $E$ identified by the IN arguments, or attacked by (an argument in) $E$. Observe that the following follows straightforwardly from Definition 6 and Theorem 1.

Proposition 1 Let $\Delta=(\mathcal{A}, \mathcal{R})$, and for $s \in$ admissible, complete, grounded, preferred, stable $\}$, let $E$ be an $s$ extension of $\Delta$. Then there exists an $s$ labelling $\mathcal{L}$ of $\Delta$ such that $\operatorname{in}(\mathcal{L})=E$, and out $(\mathcal{L})=(E+) \cup(E-)$.

Note also, that we can say that the arguments labelled OUT or UNDEC are potentially rejected arguments in the sense that if these arguments are OUT or UNDEC in all other $s$ labellings, then they are said to be rejected as defined in Definition 4.

\subsubsection{Generalising Dung's Theory with Collective Attacks}

Recent works generalise binary attacks to allow for attacks between sets of arguments $[18,44]$. We refer the reader to these works for motivation of this generalisation. Here, we briefly review [44], in which the attack relation is defined from sets of arguments to single arguments.

Definition 8 [DungC Framework with Collective Attacks] A DungC argumentation framework is a tuple $(\mathcal{A}, \mathcal{R})$, where $\mathcal{A}$ is a set of arguments, and $\mathcal{R} \subseteq\left(2^{\mathcal{A}} \backslash\{\emptyset\}\right) \times \mathcal{A}$.

- $x \in \mathcal{A}$ is acceptable w.r.t. $S \subseteq \mathcal{A}$ iff for all $B \subseteq \mathcal{A}$ such that $B \mathcal{R} x$, there exists a $C \subseteq S$ such that $C \mathcal{R} y$ for some $y \in B$.

- $S \subseteq \mathcal{A}$ is conflict free iff $\forall S^{\prime} \subseteq S, \forall x \in S$ it is not the case that $S^{\prime} \mathcal{R} x$

Given the above definitions of conflict free and acceptability for DungC frameworks, the extension-based semantics and status of arguments are defined in the same way as for a standard Dung framework (i.e., as in Definitions 3 and 4). Hence, one can straightforwardly see that a standard Dung framework is simply a special case of a DungC framework. If each attack originates from a singleton set of arguments, then the definitions of acceptability and conflict-free in Definition 8 coincide with those given in Definitions 2 and 3. As one would therefore expect, the fundamental results that hold for Dung frameworks are also shown to hold for DungC frameworks [44]. 


\subsection{Distinguishing Attack from Defeat}

Dung's extensional semantics may yield multiple extensions, so that one may then be faced with the problem of how to choose between conflicting credulously justified arguments that belong to at least one, but not all extensions. This of course equates with the familiar multiple extension problem in non-monotonic reasoning, illustrated by the well known flying or not flying Tweety and Nixon diamond examples [49].

One solution is to prioritise the object level rules that yield conflicting conclusions, where the rationale for prioritisation may or may not be explicit. For example, given that penguins are a subclass of birds, the specificity principle is used to prioritise the penguins don't fly rule over the birds fly rule, so yielding the single extension containing the conclusion Tweety doesn't fly. Hence, works such as [46] and [48] apply priorities to conflicting rules in the underlying logical formalisms that instantiate a Dung framework.

In what follows, we review approaches $[2,12,38]$ that augment Dung's framework so as to formalise the role of the relative strengths of arguments at the abstract level. The basic idea in all these works is that an attack by $x$ on $y$ succeeds as a defeat only if $y$ is not stronger than $x$. The justified arguments are then evaluated on the basis of the derived defeat relation, rather than the original attack relation. For example, given two symmetrically attacking arguments, $x$ claiming Tweety flies, and $y$ claiming Tweety does not fly (constructed in some instantiating logical formalism), then the specificity principle determines that $y$ is stronger than $x$, so that $x$ 's attack on $y$ does not succeed as a defeat, and we are left with only $y$ defeating $x$; only $y$ is a justified argument.

Consider also domains in which arguments may not be of the form premises and premises imply conclusion, so conclusion but rather result from the instantiation of some other argument schemes [54] that are stereotypical patterns of reasons that provide presumptive reasons for accepting the conclusions. One class of examples is where we have a reason to believe something on the basis of being told that it is true. Examples of this class of argument scheme include Argument from Expert Opinion and Argument from Witness Testimony. Suppose that Tweedledum says that he saw Alice playing croquet. This is an argument that Alice was playing croquet, which we believe to be true given no reason to discount it. Suppose, however, Tweedledee says Alice was not playing croquet. The Tweedledum and Tweedledee arguments attack each other, but given that we know Tweedledum is an entirely reliable witness, and that Tweedledee invariably disagrees with his brother on principle, without any real regard to the facts, we will deny that Tweedledee's argument is strong enough to successfully attack and so defeat the argument based on Tweedledum's evidence. This situation is very common: the arguments considered will often come from a variety of sources, and it is the perceived relative reliability of the sources that determines which attacks succeed as defeats (for example many court cases are presented with conflicting testimony, and turn on which the jury chooses to believe).

Thus far we have considered examples in which the relative strengths of arguments is used to 'resolve' symmetric attacks in order to obtain asymmetric defeats. In practical reasoning contexts, it may often be the case that asymmetric attacks fail, so that if $x$ attacks $y$ (but not vice versa) and $y$ is deemed stronger than $x$, then the attack fails, and neither argument defeats each other. This means that based on the defeat relation, $x$ and $y$ may both be evaluated as justified. This would clearly be inappropriate if accepting arguments $x$ and $y$ would allow us to conclude logically incompatible states of affairs as when arguing about what is believed to be the case. However, this may be appropriate when applying argumentation to practical reasoning. Consider value based 
argumentation over action [7] in which an argument $y$ justifying a course of action, such as going to a restaurant, is asymmetrically attacked by $x$ claiming that the restaurant is prohibitively expensive. If the value promoted by $y$ (gastronomic pleasure) is ranked higher than that promoted by $x$ (reducing expenditure), then the attack is removed and both arguments may then be justified. One accepts that the action of going to the restaurant is expensive, while still pursuing the course of action. Note too that the resolution is not forced one way or the other: whether I choose to go to the restaurant depends on my view of the relative importance of money and gastronomic pleasure, and different individuals may legitimately make different choices. Equally with different sources: some may trust Tweedledum more, some Tweedledee and others may believe there is nothing to choose between them.

In order to accommodate the idea that in many domains of argumentation there is some subjective element of choice in deciding whether or not an attack succeeds, attempts have been made to extend Dung's framework by adding some additional mechanism by which an attack may fail, even though the attacking argument cannot be rejected. We now briefly review three such efforts.

\subsection{Preference based Argumentation}

In Preference based Argumentation [2], a Dung framework is augmented with a preference ordering on $\mathcal{A}$, so that an attack by $x$ on $y$ succeeds as a defeat, only if $y$ is not strictly preferred to $x$.

Definition 9 [Preference Based Argumentation] A Preference based Argumentation Framework $(P A F)$ is a tuple $(\mathcal{A}, \mathcal{R}, \mathcal{P})$, where $\mathcal{A}$ is a set of arguments, $\mathcal{R} \subseteq \mathcal{A} \times \mathcal{A}$, and $\mathcal{P}$ is a preordering on $\mathcal{A} \times \mathcal{A}$.

Let $\gg_{\mathcal{P}}$ denote the strict ordering associated with $\mathcal{P}$, i.e., $y \gg_{\mathcal{P}} x$ iff $(y, x) \in \mathcal{P}$ and $(x, y) \notin \mathcal{P}$. Then:

- $\forall x, y \in \mathcal{A}, x$ defeats $y$ iff $x \mathcal{R} y$ and not $\left(y \gg_{\mathcal{P}} x\right)^{3}$.

- For $s \in\{$ admissible, complete, preferred, stable, grounded $\}, E$ is an $s$ extension of $(\mathcal{A}, \mathcal{R}, \mathcal{P})$ iff $E$ is an $s$ extension of the Dung framework $(\mathcal{A}$, defeat $)$.

The justified arguments of a $P A F(\mathcal{A}, \mathcal{R}, \mathcal{P})$ are therefore the justified arguments of the Dung framework $(\mathcal{A}$, defeat $)$. For example, consider two individuals $\mathbf{P}$ and $\mathbf{O}$ exchanging arguments $a, b$ about the weather forecast:

$\mathbf{P}_{1}$ : "Today will be dry in London since the BBC forecast sunshine" $=a$

$\mathbf{O}_{1}$ : "Today will be wet in London since CNN forecast rain" $=b$

$a$ and $b$ claim contradictory conclusions and so attack each other. Under Dung's preferred semantics, there are two extensions: $\{a\}$ and $\{b\}$. Suppose that $a$ is preferred to $b$ because the BBC are deemed more trustworthy than CNN. We have the $P A F$ :

$$
(\mathcal{A}=\{a, b\}, \mathcal{R}=\{(a, b),(b, a)\}, \mathcal{P}=\{(a, b)\})
$$

Since $a \gg_{\mathcal{P}} b$, then only $a$ defeats $b$. We thus obtain the single preferred extension $\{a\}$ of the Dung framework $(\mathcal{A}=\{a, b\}$, defeat $=\{(a, b)\})$.

\footnotetext{
${ }^{3}$ Notice that contrary to recent convention, [2] adopt the terminology in reverse, so that $\mathcal{R}$ is referred to as a defeat relation, and $x$ attacks $y$ iff $x \mathcal{R} y$ and not $\left(y \gg_{\mathcal{P}} x\right)$.
} 


\subsection{Value based Argumentation}

The preference relation in $P A F \mathrm{~s}$ is entirely abstract. Value based Argumentation [12] give more content to the notion of preferences, by relating the strength of arguments to the values promoted by accepting them; for example an argument to raise taxes is that it would promote equality, and an argument to cut taxes is that it would promote enterprise. Note that preferences over values are subjective, and depend on the person or persons, i.e., the audience, to whom the argument is addressed. Which argument is accepted will depend on whether a given audience prefers equality to enterprise, or vice versa. Hence, a Value based Argumentation Framework (VAF) extends Dung's framework to include a set of values, a function mapping arguments to these values and a set of audiences (i.e., a set of total orderings on these values). An argument $x$ defeats $y$ w.r.t. an audience $a$, if $x$ attacks $y$, and a does not rank the value promoted by $y$ higher than the value promoted by $x$. The extensions of a VAF are then the extensions of the Dung framework defined by the defeat relation.

\section{Definition 10 [Value Based Argumentation]}

- A Value based Argumentation Framework is a 5-tuple $(\mathcal{A}, \mathcal{R}, V$,val,P) where val is a function from $\mathcal{A}$ to a non-empty set of values $V$, and $P$ is a set $\left\{a_{1}, \ldots, a_{n}\right\}$, where each $\mathrm{a}_{i}$ names a total ordering (audience) $>\mathrm{a}_{i}$ on $V \times V$.

- An audience specific $V A F(a V A F)$ is a 5-tuple $(\mathcal{A}, \mathcal{R}, V$,val,a) where a $\in P$.

- Given an $\operatorname{aVAF}(\mathcal{A}, \mathcal{R}, V, v a l, \mathrm{a}), \forall x, y \in \mathcal{A}$ : $x$ defeats $_{\mathrm{a}} y$ iff $x \mathcal{R} y$, and it is not the case that $\operatorname{val}(y)>_{\mathrm{a}} \operatorname{val}(x)$.

- For $s \in\{$ admissible, complete, preferred, stable, grounded $\}, E$ is an $s$ extension of $(\mathcal{A}, \mathcal{R}, V, v a l, a)$ iff $E$ is an $s$ extension of the Dung framework $\left(\mathcal{A}\right.$, defeat $\left._{\mathrm{a}}\right)$.

The justified arguments of an $\operatorname{aVAF}(\mathcal{A}, \mathcal{R}, V, v a l, \mathrm{a})$ are therefore the justified arguments of the Dung framework $\left(\mathcal{A}\right.$, defeat $\left._{\mathrm{a}}\right)$. For example, consider the $a V A F$ consisting of arguments $y$ and $x$, where $x \mathcal{R} y, \operatorname{val}(y)=$ 'gastronomic_pleasure', $\operatorname{val}(x)=$ 'financial_prudence', and audience a orders the former value higher than the latter (recall the example in Section 2.2). Then neither argument defeat $t_{\mathrm{a}}$ each other, and so both are sceptically justified arguments of $\left(\mathcal{A}\right.$, defeat $\left._{\mathrm{a}}\right)$ (under all of the Dung semantics).

\subsection{Extended Argumentation}

In $P A F \mathrm{~s}$ and $V A F \mathrm{~s}$, preference orderings and values are applied to generate a defeat relation which is a subset of the attack relation containing only those attacks that are successful. In Extended Argumentation [38], this is achieved by directly attacking attacks with arguments, so that if $x$ attacks $y$, and $z$ attacks the attack from $x$ to $y$, then $z$ is interpreted as claiming that $y$ is stronger than $x$. The rationale for concluding that $y$ is stronger than $x$ is thus itself now part of the domain of discourse, and is encoded as an $\operatorname{argument} z$ in the object-level framework, where the rationale may be based on preferences, values, sources, or any other reason. One can also therefore account for the fact that preferences may vary according to context, and because information sources may disagree as to the criteria by which the strengths of arguments should be valuated, or the valuations assigned for a given criterion. In other words, one can account for reasoning and indeed arguing about, as well as with, defeasible and possibly conflicting information about the relative strengths of arguments. Consider Section 2.3's extended 
dialogue about the weather:

$\mathbf{P}_{1}$ : "Today will be dry in London since the BBC forecast sunshine" $=a$

$\mathbf{O}_{1}$ : "Today will be wet in London since $\mathrm{CNN}$ forecast rain" $=b$

$\mathbf{P}_{2}$ : "But the BBC are more trustworthy than $\mathrm{CNN}$ " $=c$

$\mathbf{O}_{2}$ : "However, statistics show that $\mathrm{CNN}$ are more accurate than the $\mathrm{BBC}$ " $=d$

$\mathbf{O}_{3}$ : "And a statistical comparison is more rational than a comparison based on instincts about relative trustworthiness" $=e$

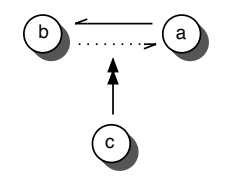

a)

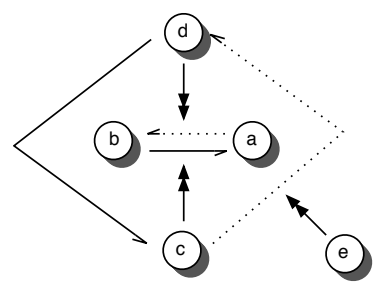

c)



b)

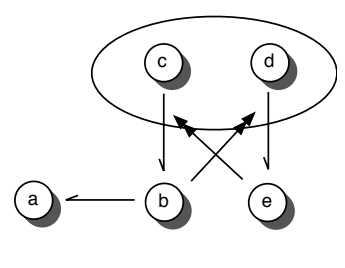

d)

Figure 2: Motivating Extended Argumentation Frameworks

In an Extended Argumentation Framework $(E A F), c$ is an argument claiming that $a$ is stronger than $b$, and so attacks $b$ 's attack on $a$. Hence, $b$ 's attack on $a$ does not succeed as a defeat, and we are left only with $a$ successfully attacking (defeating) $b$ (see Figure 2a in which we introduce the notation $y \rightarrow x$ for an attack, and $z \rightarrow(y \rightarrow x)$ for an attack on an attack). Now $\{c, a\}$ is the only preferred extension and so $a$ is sceptically justified. $d$ claims $b$ is preferred to $a$ and so attacks $a$ 's attack on $b$. Now $\{c, a\}$ and $\{d, b\}$ are preferred since the choice between $a$ and $b$ is unresolved. Notice that since $c$ and $d$ make contradictory claims about the relative strengths of $a$ and $b, c$ and $d$ attack each other (Figure 2b)). However $e$ then attacks the attack from $c$ to $d$ (Figure 2c)), and so $d$ defeats $c, b$ defeats $a$, and the discussion concludes in favour of $b(\{e, d, b\}$ is the single preferred extension). EAFs thus extend Dung frameworks with a second attack relation $\mathcal{D}$ from arguments to attacks:

Definition 11 [Extended Argumentation Framework] An Extended Argumentation Framework is a tuple $(\mathcal{A}, \mathcal{R}, \mathcal{D})$, where $\mathcal{A}$ is a set of arguments, $\mathcal{R} \subseteq \mathcal{A} \times \mathcal{A}$, and:

- $\mathcal{D} \subseteq \mathcal{A} \times \mathcal{R}$

- If $(z,(x, y)),\left(z^{\prime},(y, x)\right) \in \mathcal{D}$ then $\left(z, z^{\prime}\right),\left(z^{\prime}, z\right) \in \mathcal{R}$ 
The notion of a successful attack, henceforth referred to as a defeat, is then parameterised w.r.t. preferences specified by some given set $S$ of arguments:

Definition 12 [Defeat for $\boldsymbol{E A F}$ ] $y$ defeats $_{S} x$, denoted $y \rightarrow^{S} x$, iff $(y, x) \in \mathcal{R}$ and $\neg \exists z \in S$ s.t. $(z,(y, x)) \in S$.

In the weather example, $a$ defeats $_{\emptyset} b$ but $a$ does not defeat ${ }_{\{d\}} b$. A conflict free set of arguments is then defined to account for the case where $y$ asymmetrically attacks $x$, but given a preference for $x$ over $y$, both may appear in a conflict free set and hence an extension (as in [12]). Notice that a conflict free set does not admit arguments that symmetrically attack, irrespective of the preference arguments contained.

Definition 13 [Conflict free for $\boldsymbol{E A F}$ ] $S$ is conflict free iff $\forall x, y \in S$ : if $(y, x) \in \mathcal{R}$ then $(x, y) \notin \mathcal{R}$, and $\exists z \in S$ s.t. $(z,(y, x)) \in \mathcal{D}$.

The acceptability of an argument $x$ w.r.t. a set $S$ is now defined for an $E A F$. The basic idea is that for any attacker $y$ of $x$, a reinstating attack $z \rightarrow y$ from $z \in S$ must itself be reinstated against $\mathcal{D}$ attacks on $z \rightarrow y$. The definition is motivated in more detail in [38] and requires the notion of a reinstatement set for a defeat ${ }^{4}$.

Definition 14 [Reinstatement set] Let $S \subseteq \mathcal{A}$ in $(\mathcal{A}, \mathcal{R}, \mathcal{D})$. Let $R_{S}=\left\{x_{1} \rightarrow^{S}\right.$ $\left.y_{1}, \ldots, x_{n} \rightarrow{ }^{S} y_{n}\right\}$ where for $i=1 \ldots n, x_{i} \in S$. Then $R_{S}$ is a reinstatement set for $a \rightarrow S$, iff

- $a \rightarrow^{S} b \in R_{S}$, and

- $\forall x \rightarrow{ }^{S} y \in R_{S}, \forall y^{\prime}$ s.t. $\left(y^{\prime},(x, y)\right) \in \mathcal{D}, \exists x^{\prime} \rightarrow^{S} y^{\prime} \in R_{S}$

Definition 15 [Acceptability for $\boldsymbol{E A F} \mathbf{s}$ ] $x$ is acceptable w.r.t. $S \subseteq \mathcal{A}$ iff $\forall y$ s.t. $y \rightarrow^{S}$ $x, \exists z \in S$ s.t. $z \rightarrow^{S} y$ and there is a reinstatement set for $z \rightarrow^{S} y$.

In Figure 2d), $a$ is acceptable w.r.t. $S$. We have $b \rightarrow^{S} a, c \rightarrow^{S} b$, and there is a reinstatement set $\left\{c \rightarrow^{S} b, d \rightarrow^{S} e\right\}$ for $c \rightarrow^{S} b$. Note that if we had $f \rightarrow(d \rightarrow e)$, and no argument in $S$ defeating $f$, there would be no reinstatement set, and $a$ would not be acceptable w.r.t. $S$.

Given the definitions of conflict free and acceptability for $E A F \mathrm{~s}$, admissible, complete, preferred and stable semantics for $E A F$ s are now defined as for Dung argumentation frameworks in Definition 3 (except that $x$ defeats $_{S} y$ replaces $(x, y) \in \mathcal{R}$ in the definition of stable extensions). In [38] it is shown that EAFs inherit many of the results that hold for Dung frameworks (e.g., Dung's fundamental lemma). However, an $E A F$ 's characteristic function is not in general monotonic. Recall that the grounded extension of a Dung framework is its minimal (under set inclusion) complete extension. A complete extension can equivalently be characterised as a fix point of a Dung framework's characteristic function $F$ which given a set of arguments $S$ returns the arguments $S^{\prime}$ acceptable w.r.t. $S\left(S^{\prime}=F(S)\right.$ ). Since $F$ is monotonic ( $S \subseteq S^{\prime}$ implies $F(S) \subseteq F\left(S^{\prime}\right)$ ) a least fixed point characterising the grounded extension can be guaranteed. However, the characteristic function $F$ of an $E A F$ is not monotonic, so that the grounded extension of a finitary $E A F$ (in which arguments and attacks are attacked by at most a finite number of arguments) is defined by iteration of the function, that starting with the empty set, does yield a monotonically increasing sequence. Let $G^{0}=$ $\emptyset, G^{i+1}=F\left(G^{i}\right)$. The grounded extension of an $E A F$ is defined as $\bigcup_{i=0}^{\infty} G^{i}$.

\footnotetext{
${ }^{4}$ This ensures satisfaction of an intuitive requirement (Dung's fundamental lemma [27]) on what it means for an argument to be acceptable w.r.t. an admissible set $S$, viz. that if $x$ is acceptable with respect to $S$, then $S \cup\{x\}$ is admissible
} 


\section{Formalising Abstract Argumentation in Metalevel Ar- gumentation Frameworks}

In this section we formalise Metalevel Argumentation Frameworks that essentially augment a Dung framework $(\mathcal{A}, \mathcal{R})$ with a language for representing the claims of arguments in $\mathcal{A}$, and constraints on the attack relation $\mathcal{R}$ that account for the arguments' claims. The arguments in $\mathcal{A}$ are arguments claiming statements about object level abstract argumentation frameworks, and the constraints on $\mathcal{R}$ essentially characterise the reasoning by which one evaluates the justified arguments of the object level framework. We then show how the varieties of abstract argumentation reviewed in Section 2 can be formalised as instances of metalevel argumentation.

\subsection{Introducing Metalevel Argumentation}

Section 2's review of abstract argumentation described how, in general, establishing that an argument $x$ is justified under a semantics $s$ is based on evaluation of the acceptability of $x$ w.r.t. sets of arguments. The acceptability of $x$ w.r.t. some subset $S$ of $\mathcal{A}$, hinges on whether attacks of the form $y \mathcal{R} x$ succeed as defeats. If for each such $y \mathcal{R} x, y$ is successfully attacked (defeated) by some $z \in S$, then $z$ effectively undermines the success of the attack from $y$ to $x$; $z$ can be said to reinstate $x$. The rules defining legal labelling assignments for Dung frameworks correspond intuitively to the extension-based use of this reinstatement principle:

R1 $x$ is legally IN (i.e., $x$ is in an admissible extension) in an $s$ labelling (credulously justified under the semantics $s$ ) iff every attack $y \mathcal{R} x$ on $x$ fails.

$\mathrm{R} 2$ An attack $y \mathcal{R} x$ fails if $y$ is OUT (i.e., $y$ is attacked by a reinstating $z$ that is IN)

R3 $x$ is legally OUT (potentially rejected under the semantics $s$ ) if at least one attack $y \mathcal{R} x$ succeeds (i.e., $y$ in IN)

Given a Dung framework $\Delta=(\mathcal{A}, \mathcal{R})$, a statement asserting the existence of an argument $x \in \mathcal{A}$, and its purported membership of an admissible extension of $\Delta$, constitutes a metalevel argument $\xi$ claiming ' $x$ is justified'. That is to say, $\xi$ is an argument of the form 'there is an $x \in \mathcal{A}$ that is an admissible extension of $\Delta$, and so $x$ is justified'. Note that logics for asserting statements of this kind have been proposed in $[19,35,55]$ and will be briefly reviewed in Section 5 .

MR1 The existence of an attack $y \mathcal{R} x$, constitutes a metalevel argument $\alpha$, that claims that ' $y$ successfully attacks and so defeats $x$ '. Since the justified status of $x$ is challenged by a defeat on $x$, we therefore have a metalevel attack from $\alpha$ to $\xi$. Hence, a metalevel attack on $\alpha$, challenging $y$ 's defeat of $x$ and so reinstating $\xi$, characterises the object level reinstatement of $x$.

MR2 $y$ does not defeat $x$ if $y$ is rejected (i.e., R2). Hence, in the metalevel, $\alpha$ is attacked by an argument $\tau$ claiming that ' $y$ is rejected'. Thus $\tau$ reinstates $\xi$.

MR3 $y$ defeats $x$ if $y$ is justified. Thus, in the metalevel, the argument $\psi$ claiming that ' $y$ is justified' attacks the argument $\tau$ claiming that ' $y$ is rejected', so reinstating the argument $\alpha$, that claims that ' $y$ defeats $x$ '. 
The metalevel arguments and their attacks are illustrated in Figure 3a. Figure 3b shows the metalevel argumentation corresponding to the object level reinstatement of $x$, by some $z$ that attacks $y$. At the metalevel, we have an argument claiming ' $z$ is justified' that reinstates the metalevel argument claiming $z$ defeats $y$, in turn reinstating $\tau$ claiming ' $y$ is rejected', which in turn reinstates $\xi$ claiming ' $x$ is justified'. Finally, Figure $3 \mathrm{c}$ shows the metalevel argumentation corresponding to an object level symmetric attack between arguments $x$ and $y(x \mathcal{R} y$ and $y \mathcal{R} x)$. In this case the metalevel argumentation characterises the object level reinstatement of $x$ by $x$ itself.


Figure 3: Meta-level arguments and their attacks

In our description of metalevel arguments, we have assumed their classification according to the nature of the claims they make about an object level framework, and based on this classification the attacks amongst these metalevel arguments. This suggests the more general notion of a structured argumentation framework, whereby one augments a Dung framework $(\mathcal{A}, \mathcal{R})$ to include a mapping $\mathcal{C}$ from $\operatorname{arguments}$ in $\mathcal{A}$ to claims specified in some language $\mathcal{L}$, and based on these claims a set of constraints that are thus imposed on $\mathcal{R}$.

Definition 16 [Structured Argumentation Frameworks] A Structured Argumentation Framework $(S A F)$ is a tuple $\Delta=(\mathcal{A}, \mathcal{R}, \mathcal{C}, \mathcal{L}, \mathcal{D})$, where:

- $(\mathcal{A}, \mathcal{R})$ is a Dung argumentation framework

- $\mathcal{L}$ is a claim language

- $\mathcal{C}$ is a claim function mapping arguments in $\mathcal{A}$ to sets of wff in $\mathcal{L}$ 
- $\mathcal{D}$ is a set of constraint rules on $\mathcal{R}$ of the form:

$$
\text { if } l \in \mathcal{C}(\alpha) \text { and } l^{\prime} \in \mathcal{C}(\beta) \text { then }(\alpha, \beta) \in \mathcal{R}
$$

where $\alpha, \beta \in \mathcal{A}$, and $l, l^{\prime}$ are wff of $\mathcal{L}$.

In general, we say that an attack relation $\mathcal{R}$ is defined by $\mathcal{D}$ if whenever $(\alpha, \beta) \in$ $\mathcal{R}$ then the claims of $\alpha$ and $\beta$ satisfy the antecedent of some constraint rule in $\mathcal{D}$.

For $s \in\{$ admissible, complete, grounded, preferred, stable $\}$, we say that $E$ is an $s$ extension of $\Delta$ iff $E$ is an $s$ extension of $(\mathcal{A}, \mathcal{R})$, and $\alpha \in \mathcal{A}$ is a justified argument of $\Delta$ iff $\alpha$ is a justified $\operatorname{argument}$ of $(\mathcal{A}, \mathcal{R})$.

We now identify a special class of $S A F \mathrm{~s}$ - Metalevel Argumentation Frameworks ( $M A F \mathrm{~s}$ ) — by specifying a language $\mathcal{L}$ whose wff are built from constants, sets of constants, sets of pairs of constants (where these constants may name arguments and or values), and predicates of the form justified, rejected, defeat e.t.c. Claims in this language will thus refer to the properties of arguments, values and preferences in an object level framework.

Definition 17 [Metalevel Argumentation Frameworks] A Metalevel Argumentation Framework $(M A F)$ is a $S A F \Delta_{\mathcal{M}}=\left(\mathcal{A}, \mathcal{R}, \mathcal{C}, \mathcal{L}_{\mathcal{M}}, \mathcal{D}\right)$, where $\mathcal{L}_{\mathcal{M}}$ consists of a countable set of constant symbols and the set of predicates:

$\{$ justified, defeat, rejected, preferred, val, val_pref, audience $\}$.

The set of wff of $\mathcal{L}_{\mathcal{M}}$ is defined by the following BNF:

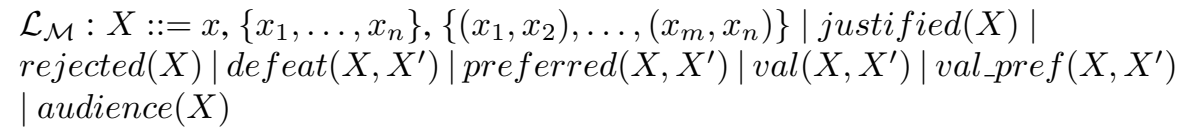

where $x, x_{i}$ ranges over the constant symbols.

In the following sections we show how the varieties of abstract argumentation reviewed in Section 2 can be formalised as instances of argumentation in a $M A F$. In each case, the constraints in $\mathcal{D}$ characterise evaluation of the justified arguments in the object level framework.

\subsection{Formalising Dung's abstract argumentation theory in Metalevel Argumentation Frameworks}

A MAF's formalisation of Dung argumentation consists of: i) metalevel arguments whose construction is based on the existence of object level attacks, and so claim defeats between object level arguments; ii) metalevel arguments whose construction is based on the existence of object level arguments and their purported membership or non-membership of admissible extensions, and that claim that the object level arguments are justified, respectively rejected; iii) constraints on the metalevel attack relation that capture MR1 - MR3 in Section 3.1:

Definition 18 [Metalevel Dung Argumentation] A Dung $M A F$ is a tuple $\left(\mathcal{A}_{\mathcal{M}}\right.$, $\left.\mathcal{R}_{\mathcal{M}}, \mathcal{C}, \mathcal{L}_{M}, \mathcal{D}_{d}\right)$, where: $\mathcal{D}_{d}=\{$

$$
\begin{aligned}
\{D 1 & : \text { if } \mathcal{C}(\alpha)=\operatorname{defeat}(Y, X) \text { and } \mathcal{C}(\beta)=\text { justified }(X) \text { then }(\alpha, \beta) \in \mathcal{R}_{\mathcal{M}} \\
D 2 & : \text { if } \mathcal{C}(\alpha)=\operatorname{defeat}(Y, X) \text { and } \mathcal{C}(\beta)=\operatorname{rejected}(Y) \text { then }(\beta, \alpha) \in \mathcal{R}_{\mathcal{M}}
\end{aligned}
$$




$$
\left.D 3: \text { if } \mathcal{C}(\alpha)=j u s t i f i e d(X) \text { and } \mathcal{C}(\beta)=\operatorname{rejected}(X) \text { then }(\alpha, \beta) \in \mathcal{R}_{\mathcal{M}}\right\}
$$

Definition 19 [Mapping Dung Frameworks to Metalevel Frameworks] A Dung MAF $\Delta_{M}=\left(\mathcal{A}_{\mathcal{M}}, \mathcal{R}_{\mathcal{M}}, \mathcal{C}, \mathcal{L}_{\mathcal{M}}, \mathcal{D}_{d}\right)$ is said to be a metalevel formulation of the Dung $A F \Delta=(\mathcal{A}, \mathcal{R})$ iff:

- $\lceil x\rceil$ is a constant in $\mathcal{L}_{M}$ iff $x \in \mathcal{A}^{5}$

- $\mathcal{A}_{\mathcal{M}}$ is the union of the disjoint sets $\mathcal{A}_{\mathcal{M} 1}, \mathcal{A}_{\mathcal{M} 2}, \mathcal{A}_{\mathcal{M} 3}$, where:

1. $\alpha \in \mathcal{A}_{\mathcal{M} 1}, \mathcal{C}(\alpha)=$ justified $(\lceil x\rceil)$ iff $x \in \mathcal{A}$

2. $\beta \in \mathcal{A}_{\mathcal{M} 2}, \mathcal{C}(\beta)=\operatorname{rejected}(\lceil x\rceil)$ iff $x \in \mathcal{A}$

3. $\gamma \in \mathcal{A}_{\mathcal{M} 3}, \mathcal{C}(\gamma)=\operatorname{defeat}(\lceil y\rceil,\lceil x\rceil)$ iff $(y, x) \in \mathcal{R}$

- $\mathcal{R}_{\mathcal{M}}$ is defined by $\mathcal{D}_{d}$.

Notation 2 Henceforth, we may as an abuse of notation refer to a metalevel argument $\alpha$ by a shorthand reference to the claim that $\alpha$ makes:

- If $\mathcal{C}(\alpha)=$ justified $(\lceil x\rceil)$ we write $(j-x)$ to refer to $\alpha$.

- If $\mathcal{C}(\alpha)=\operatorname{rejected}(\lceil x\rceil)$ we write $(r-x)$ to refer to $\alpha$.

- If $\mathcal{C}(\alpha)=\operatorname{defeat}(\lceil y\rceil,\lceil x\rceil)$ we write $(y \operatorname{def} x)$ to refer to $\alpha$.

Given a Dung framework $\Delta=(\mathcal{A}, \mathcal{R})$, one can evaluate the justified arguments of $\Delta$ by evaluating the justified arguments of the Dung $M A F \Delta_{M}$.

Theorem 2 Let $\Delta_{M}=\left(\mathcal{A}_{\mathcal{M}}, \mathcal{R}_{\mathcal{M}}, \mathcal{C}, \mathcal{L}_{\mathcal{M}}, \mathcal{D}_{d}\right)$ be the $M A F$ of a Dung framework $(\mathcal{A}, \mathcal{R})$. Then for $s \in\{$ complete, grounded, preferred, stable $\},(j-x) \in \mathcal{A}_{\mathcal{M}}$ is a credulously, respectively sceptically, justified argument of $\Delta_{M}$ under the $s$ semantics, iff $x \in \mathcal{A}$ is a credulously, respectively sceptically, justified argument of $\Delta$ under the $s$ semantics.

Note the extra expressivity that results from the metalevel formulation of a Dung framework. Given an extension $E$ of $\Delta$, the corresponding extension of $\Delta_{M}$ identifies the arguments labelled IN and OUT by a corresponding labelling for $E$.

Proposition 2 Let $\Delta_{\mathcal{M}}=\left(\mathcal{A}_{\mathcal{M}}, \mathcal{R}_{\mathcal{M}}, \mathcal{C}, \mathcal{L}_{\mathcal{M}}, \mathcal{D}_{d}\right)$ be the $M A F$ of a Dung framework $\Delta=(\mathcal{A}, \mathcal{R})$. For $s \in\{$ admissible, complete, grounded, preferred, stable $\}$ :

There exists an $s$ labelling $\mathcal{L}$ of $\Delta$ iff there exists an $s$ extension $E$ of $\Delta_{\mathcal{M}}$ such that:

1. $x \in \operatorname{in}(\mathcal{L})$ iff $(j-x) \in E$

2. $y \in \operatorname{out}(\mathcal{L})$ iff $(r-y) \in E$

To illustrate, consider Figure 4's object level Dung framework $\Delta$, and its metalevel formulation $\Delta_{M}$. Observe that $\{d, c, b\}$ is the grounded, preferred and stable extension of $\Delta$, corresponding to $\{(d$ def $e),(j-d),(r-e),(c$ def $e),(j-c),(r-a),(b \operatorname{def}$ $a),(j-b)\}$ being the grounded, preferred and stable extension of $\Delta_{M}$.

\footnotetext{
${ }^{5}$ It is standard practice to use sense quotes \lceil\rceil (also called Frege or Gödel quotes) to abbreviate metalevel representations of object level formulae.
} 


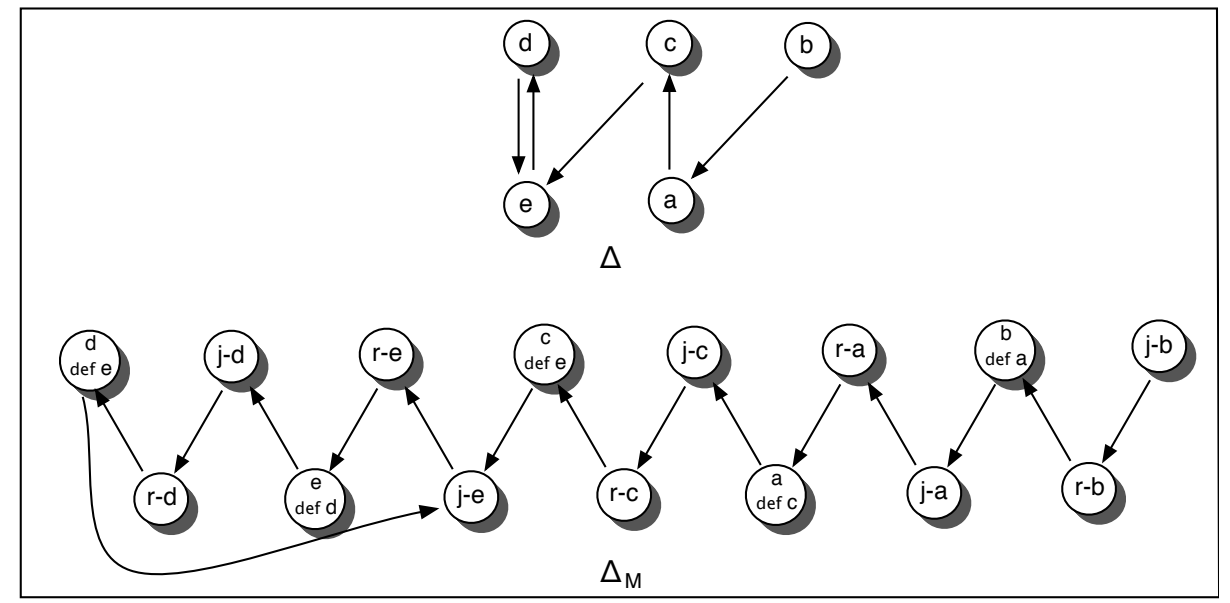

Figure 4: A Dung argumentation framework and its metalevel formulation

Metalevel argumentation allows us to formalise the various developments of Dung's argumentation theory described in Section 2, within Dung frameworks themselves, so that one can then apply the considerable body of results and techniques for Dung frameworks, to these extensions. In the following section we describe the metalevel formulation of [44]'s generalisation of Dung frameworks to include collective attacks.

\subsection{Formalising Collective Attacks in Meta-level Argumentation Frameworks}

As one would expect, the existence of a collective attack $B \mathcal{R} x$ constitutes an argument $\alpha$ claiming ' $B$ defeats $x$ ', and for each $y \in B$, an argument claiming ' $y$ is rejected' attacks $\alpha$.

Definition 20 [Metalevel Dung Argumentation with Collective Attacks] A DungC $M A F$ is a tuple $\left(\mathcal{A}_{\mathcal{M}}, \mathcal{R}_{\mathcal{M}}, \mathcal{C}, \mathcal{L}_{\mathcal{M}}, \mathcal{D}_{d c}\right)$, where $\mathcal{D}_{d c}=$

$$
\begin{aligned}
\{D 1 & : \text { if } \mathcal{C}(\alpha)=\operatorname{defeat}(Y, X) \text { and } \mathcal{C}(\beta)=\text { justified }(X) \text { then }(\alpha, \beta) \in \mathcal{R}_{\mathcal{M}} \\
D 2 & : \text { if } \mathcal{C}(\alpha)=\operatorname{defeat}(Y, X), \mathcal{C}(\beta)=\operatorname{rejected}(Z), \text { and } Z \in Y, \text { then }(\beta, \alpha) \in \mathcal{R}_{\mathcal{M}} \\
D 3 & \left.: \text { if } \mathcal{C}(\alpha)=\text { justified }(X) \text { and } \mathcal{C}(\beta)=\operatorname{rejected}(X) \text { then }(\alpha, \beta) \in \mathcal{R}_{\mathcal{M}}\right\}
\end{aligned}
$$

Definition 21 [Mapping DungC Frameworks to Metalevel Frameworks] A DungC $M A F \Delta_{M}=\left(\mathcal{A}_{\mathcal{M}}, \mathcal{R}_{\mathcal{M}}, \mathcal{C}, \mathcal{L}_{\mathcal{M}}, \mathcal{D}_{d c}\right)$ is said to be a metalevel formulation of the DungC framework $\Delta=(\mathcal{A}, \mathcal{R})$ iff:

- $\lceil x\rceil$ is a constant in $\mathcal{L}_{d c}$ iff $x \in \mathcal{A}$

- $\mathcal{A}_{\mathcal{M}}$ is the union of the disjoint sets $\mathcal{A}_{\mathcal{M} 1}, \mathcal{A}_{\mathcal{M} 2}, \mathcal{A}_{\mathcal{M} 3}$, where:

1. $\alpha \in \mathcal{A}_{\mathcal{M} 1}, \mathcal{C}_{\mathcal{M}}(\alpha)=$ justified $(\lceil x\rceil)$ iff $x \in \mathcal{A}$

2. $\beta \in \mathcal{A}_{\mathcal{M} 2}, \mathcal{C}_{\mathcal{M}}(\beta)=\operatorname{rejected}(\lceil x\rceil)$ iff $x \in \mathcal{A}$ 
3. $\gamma \in \mathcal{A}_{\mathcal{M} 3}, \mathcal{C}_{\mathcal{M}}(\gamma)=\operatorname{defeat}\left(\left\{\left\lceil y_{1}\right\rceil, \ldots,\left\lceil y_{n}\right\rceil\right\},\lceil x\rceil\right)$ iff $\left(\left\{y_{1}, \ldots, y_{n}\right\},\{x\}\right)$ $\in \mathcal{R}$

- $\mathcal{R}_{\mathcal{M}}$ is defined by $\mathcal{D}_{d c}$.

Henceforth, we employ a similar abuse of notation as in Notation 2 , writing $(j-x)$ and $(r-x)$ to refer to arguments $\alpha$ and $\beta$ that respectively claim justified $(\lceil x\rceil)$ and rejected $(\lceil x\rceil)$, and $\left(\left\{y_{1}, \ldots, y_{n}\right\}\right.$ def $\left.x\right)$ to refer to an argument claiming defeat $\left(\left\{\left\lceil y_{1}\right\rceil\right.\right.$, $\left.\left.\ldots,\left\lceil y_{n}\right\rceil\right\},\lceil x\rceil\right)$.
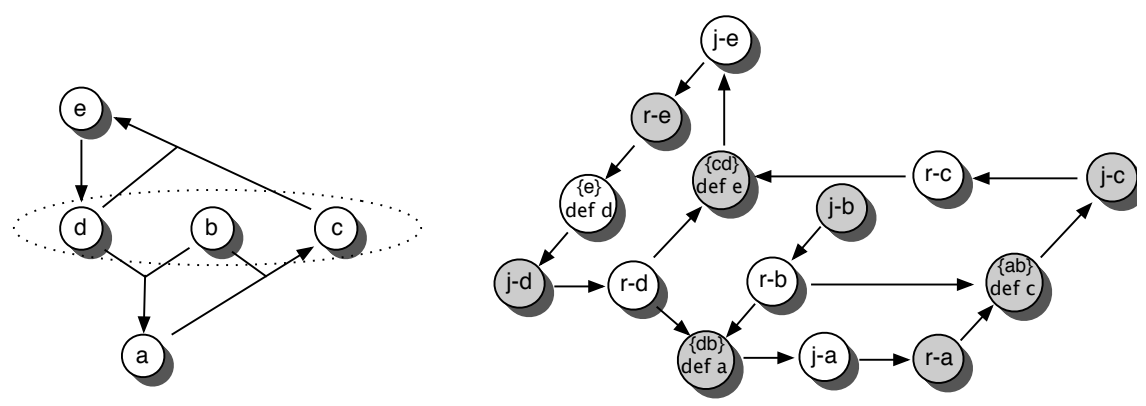

Figure 5: A DungC framework with collective attacks, and its metalevel formulation

Consider Figure 5's object level DungC framework with the following attacks:

$$
\{a, b\} \rightarrow c,\{d, b\} \rightarrow a,\{e\} \rightarrow d,\{d, c\} \rightarrow e,
$$

One can easily verify that $E 1=\{d, b, c\}$ (the encircled arguments) and $E 2=\{a, b, e\}$ are the preferred and stable extensions, and $\{b\}$ the grounded extension. Figure 5 shows the object level framework's $M A F$, and the arguments (shaded) in the preferred and stable extension $E 1^{\prime}$ corresponding to $E 1$. In general, the following correspondence holds:

Theorem 3 Let $\Delta_{M}=\left(\mathcal{A}_{\mathcal{M}}, \mathcal{R}_{\mathcal{M}}, \mathcal{C}, \mathcal{L}_{\mathcal{M}}, \mathcal{D}_{d c}\right)$ be the $M A F$ of a DungC framework $\Delta=(\mathcal{A}, \mathcal{R})$. Then for $s \in\{$ complete, grounded, preferred, stable $\},(j-x) \in \mathcal{A}_{\mathcal{M}}$ is a credulously, respectively sceptically, justified argument of $\Delta_{M}$ under the $s$ semantics, iff $x \in \mathcal{A}$ is a credulously, respectively sceptically, justified argument of $\Delta$ under the $s$ semantics.

\subsection{Formalising Preference Based Argumentation in Meta-level Ar- gumentation Frameworks}

As discussed in Sections 2.3, 2.4 and 2.5, PAFs, VAFs and EAFs provide additional information which enable, when evaluating the framework, to say that an attack fails to succeed as a defeat, even though the attacking argument is justified. In terms of metalevel argumentation, this additional information is the source of additional arguments which can be used to attack arguments of the form ( $x$ def $y$ ). In a PAF, this extra information is simply a preference ordering on the arguments in the framework.

Given an object level $P A F$, the existence of a strict preference $x \gg_{\mathcal{P}} y$ constitutes a metalevel argument claiming that ' $x$ is strictly preferred to $y$ '. In addition to MR2 
in Section 3.1, we thus have the additional following metalevel characterisation of the object level reasoning that determines the justified arguments of a $P A F$ :

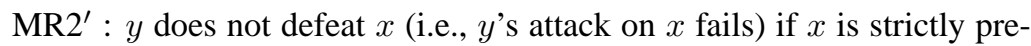
ferred to $y$. Hence, in the metalevel, $\alpha$ claiming ' $y$ defeats $x$ ' is attacked by an argument $\rho$ claiming that ' $x$ is strictly preferred to $y$ '. Thus $\rho$ reinstates $\xi$ claiming ' $x$ is justified'.

Definition 22 [Metalevel Preference based Argumentation] A $P-M A F$ is a tuple $\left(\mathcal{A}_{\mathcal{M}}, \mathcal{R}_{\mathcal{M}}, \mathcal{C}, \mathcal{L}_{\mathcal{M}}, \mathcal{D}_{p}\right)$, where $\mathcal{D}_{p}=\mathcal{D}_{d} \cup$

$\left\{\mathrm{D} 4:\right.$ if $\mathcal{C}(\alpha)=\operatorname{defeat}(Y, X)$ and $\mathcal{C}(\beta)=$ s_preferred $_{\text {pref }}(X, Y)$ then $\left.(\beta, \alpha) \in \mathcal{R}_{\mathcal{M}}\right\}$

Definition 23 [Mapping PAFs to Metalevel Frameworks] A $P-M A F \Delta_{M}=\left(\mathcal{A}_{\mathcal{M}}\right.$, $\left.\mathcal{R}_{\mathcal{M}}, \mathcal{C}, \mathcal{L}_{\mathcal{M}}, \mathcal{D}_{p}\right)$ is said to be a metalevel formulation of the $\operatorname{PAF} \Delta=(\mathcal{A}, \mathcal{R}, \mathcal{P})$ iff:

- $\lceil x\rceil$ is a constant in $\mathcal{L}_{\mathcal{M}}$ iff $x \in \mathcal{A}$

- $\mathcal{A}_{\mathcal{M}}$ is the union of the disjoint sets $\mathcal{A}_{\mathcal{M} 1}, \mathcal{A}_{\mathcal{M} 2}, \mathcal{A}_{\mathcal{M} 3}, \mathcal{A}_{\mathcal{M} 4}$, where $\mathcal{A}_{\mathcal{M} 1}$, $\mathcal{A}_{\mathcal{M} 2}$ and $\mathcal{A}_{\mathcal{M} 3}$ are defined as in Definition 19, and:

4. $\delta \in \mathcal{A}_{\mathcal{M} 4}, \mathcal{C}(\delta)=s_{-}$preferred $(\lceil x\rceil,\lceil y\rceil)$ iff $x \gg_{\mathcal{P}} y$

(from hereon, we may write $(x P y)$ to refer to an argument of the form $\delta$ ).

- $\mathcal{R}_{\mathcal{M}}$ is defined by $\mathcal{D}_{p}$.

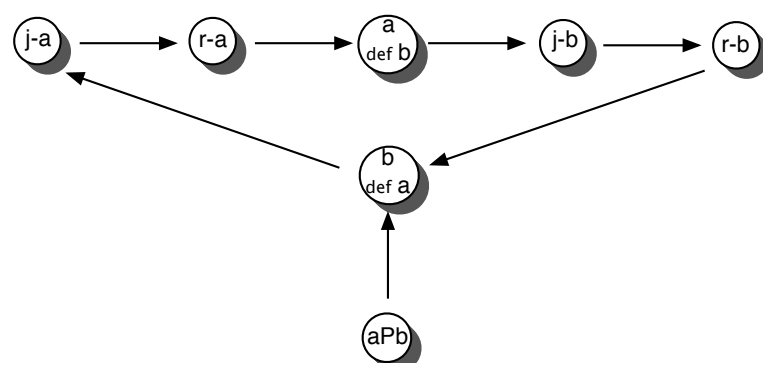

Figure 6: A PAF formulated as a metalevel framework

The metalevel formulation of the weather example $P A F(\mathcal{A}=\{a, b\}, \mathcal{R}=\{(a, b),(b, a)\}$, $\mathcal{P}=\{(a, b)\})$, is shown in Figure 6. $E^{\prime}=\{(j-a),(a \operatorname{def} b),(r-b),(a P b)\}$ is the single grounded/preferred/stable extension of the metalevel framework, corresponding to the single grounded/preferred/stable extension $\{a\}$ of the object level $P A F$.

Theorem 4 Let $\Delta_{M}=\left(\mathcal{A}_{\mathcal{M}}, \mathcal{R}_{\mathcal{M}}, \mathcal{C}, \mathcal{L}_{\mathcal{M}}, \mathcal{D}_{p}\right)$ be the $P-M A F$ of a $P A F \Delta=$ $(\mathcal{A}, \mathcal{R}, \mathcal{P})$. Then for $s \in\{$ complete, grounded, preferred, stable $\},(j-x) \in \mathcal{A}_{\mathcal{M}}$ is a credulously, respectively sceptically, justified argument of $\Delta_{M}$ under the $s$ semantics, iff $x \in \mathcal{A}$ is a credulously, respectively sceptically, justified argument of $\Delta$ under the $s$ semantics.

Note that our weather forecast example illustrates resolution of an argumentation framework obtained by replacing symmetric attacks with asymmetric attacks. Properties relating frameworks and their resolutions have been studied in [9] and [35]. Our 
metalevel formulation of $P A F$ s provides a general setting for further formal study of resolution semantics [9], whereby the reasoning by which an object-level framework's resolutions are obtained is now modelled in terms of argumentation within its metalevel formulation.

Secondly, note that since there is a single preference relation, arguments of the form $a P b$ will not attack one another, nor be attacked by any other argument. If the preference relation supplies a total order, the corresponding $P-M A F$ will contain no cycles and so have a non-empty grounded extension, and a unique non empty preferred and stable extension. If, however, the preference order is a partial order, the preferred and grounded extensions may not coincide: there may be multiple preferred extensions, corresponding to choices between mutually attacking arguments between which no preference is expressed in the preference relation. Provided, however, that there is a preference between at least one pair of arguments in every cycle, the grounded and preferred extensions will be non-empty.

\subsection{Formalising Value Based Argumentation in Meta-level Argu- mentation Frameworks}

In $V A F$ s we have rather more additional information: a set of values, a function mapping arguments to values, and a set of audiences representing totally ordered preference relations on values. The metalevel characterisation of the object level reasoning applied to determine the justified arguments of an audience specific $V A F$ ( $a V A F)$, augments MR2 in Section 3.1 as follows:

MR2.1 $y$ does not defeat $x$ (i.e., $y$ 's attack on $x$ fails) if $x$ 's value is preferred to $y$ 's value. Hence, in the metalevel, $\alpha$ claiming ' $y$ defeats $x$ ' is attacked by $a$ value preference argument $\nu$ claiming that ' $x$ 's value is preferred to $y$ 's value'. Thus $\nu$ reinstates $\xi$ claiming ' $x$ is justified'.

MR2.2 The preference of $x$ 's value over $y$ 's value is challenged by the contrary preference. Hence, in the metalevel each $\nu$ is symmetrically attacked by the metalevel argument $\nu^{\prime}$ claiming that ' $y$ 's value is preferred to $x$ 's value'.

MR2.3 The $a V A F$ 's choice of audience (total ordering on values) endorses the pairwise value preferences specified by the total ordering. Thus, an audience constitutes a metalevel argument that claims a total ordering, and that attacks any value preference arguments that contradict the endorsed value preferences. Hence, if $\operatorname{val}(x)>_{\mathrm{a}} \operatorname{val}(y)$, then a constitutes an audience argument that attacks $\nu^{\prime}$ and thus reinstates $\nu$.

We can also represent all audiences in a $V A F$, where given $P=\left\{\mathrm{a}_{1}, \ldots, \mathrm{a}_{n}\right\}$, then each $\mathrm{a}_{i}$ constitutes an audience argument that symmetrically attacks every other audience argument, since by definition, for all $i, j$ such that $i \neq j, a_{i}$ and $a_{j}$ contradict each other on at least one value preference.

Definition 24 [Metalevel Value based Argumentation] A $V-M A F$ is a tuple $\left(\mathcal{A}_{\mathcal{M}}\right.$, $\left.\mathcal{R}_{\mathcal{M}}, \mathcal{C}, \mathcal{L}_{\mathcal{M}}, \mathcal{D}_{v}\right)$, where $\mathcal{D}_{v}=\mathcal{D}_{d} \cup$

$\left\{\right.$ D4 : if $\mathcal{C}(\alpha)=\operatorname{defeat}(Y, X)$ and $\mathcal{C}(\beta)=\operatorname{val} \_p r e f\left(\operatorname{val}(X, V), \operatorname{val}\left(Y, V^{\prime}\right)\right)$ then $(\beta, \alpha) \in \mathcal{R}_{\mathcal{M}}$ 
D5 : if $\mathcal{C}(\alpha)=$ val_pref $\left(\operatorname{val}\left(Y, V^{\prime}\right), \operatorname{val}(X, V)\right)$ and $\mathcal{C}(\beta)=\operatorname{val} \_p r e f(\operatorname{val}(X, V)$, $\left.\operatorname{val}\left(Y, V^{\prime}\right)\right)$ then $(\beta, \alpha) \in \mathcal{R}_{\mathcal{M}}$

D6 : if $\mathcal{C}(\alpha)=$ audience $(Z)$ and $\mathcal{C}(\beta)=\operatorname{val} \_$pref $\left(\operatorname{val}\left(Y, V^{\prime}\right), \operatorname{val}(X, V)\right)$, where $Z$ is a set of pairs of constants such that $\left(V, V^{\prime}\right) \in Z$, then $(\alpha, \beta) \in \mathcal{R}_{\mathcal{M}}$

D7 : if $\mathcal{C}(\beta)=\operatorname{audience}(Z)$ and $\mathcal{C}(\alpha)=\operatorname{audience}\left(Z^{\prime}\right)$, where $Z$ and $Z^{\prime}$ are sets of pairs of constants such that $\left(V, V^{\prime}\right) \in Z,\left(V^{\prime}, V\right) \in Z^{\prime}$ then $\left.(\beta, \alpha) \in \mathcal{R}_{\mathcal{M}}\right\}$

Definition 25 [Mapping $\boldsymbol{V A F s}$ to Metalevel Frameworks] A $V-M A F \Delta_{M}=\left(\mathcal{A}_{\mathcal{M}}\right.$, $\left.\mathcal{R}_{\mathcal{M}}, \mathcal{C}, \mathcal{L}_{\mathcal{M}}, \mathcal{D}_{v}\right)$ is said to be a metalevel formulation of the $V A F \Delta=(\mathcal{A}, \mathcal{R}, V$, val, $P$ ) iff:

- $\lceil x\rceil$ is a constant in $\mathcal{L}_{\mathcal{M}}$ iff $x \in \mathcal{A}$ or $x \in V$

- $\mathcal{A}_{\mathcal{M}}$ is the union of the disjoint sets $\mathcal{A}_{\mathcal{M} 1}, \mathcal{A}_{\mathcal{M} 2}, \mathcal{A}_{\mathcal{M} 3}, \mathcal{A}_{\mathcal{M} 4}, \mathcal{A}_{\mathcal{M} 5}$ where $\mathcal{A}_{\mathcal{M} 1} \ldots \mathcal{A}_{\mathcal{M} 3}$ are defined as in Definition 19, and:

4. $\nu \in \mathcal{A}_{\mathcal{M} 4}, \mathcal{C}(\nu)=\operatorname{val}_{-} \operatorname{pre} f\left(\operatorname{val}(\lceil x\rceil,\lceil v\rceil), \operatorname{val}\left(\lceil y\rceil,\left\lceil v^{\prime}\right\rceil\right)\right)$ iff $\operatorname{val}(x)=v$, $\operatorname{val}(y)=v^{\prime}$, and $v \neq v^{\prime}$ (henceforth we may write $\left(x_{v} P y_{v^{\prime}}\right)$ to refer to an argument of the form $\nu$ )

5. $\left.\epsilon \in \mathcal{A}_{\mathcal{M}_{5}}, \mathcal{C}(\epsilon)=\left\{\left(v_{1}, v_{2}\right) \ldots\left(v_{m}, v_{n}\right)\right)\right\}$ iff $\mathrm{a} \in P, \mathrm{a}=v_{1}>_{\mathrm{a}} v_{2} \ldots v_{m}>_{\mathrm{a}}$ $v_{n}$ (henceforth we may write $\left(>_{a}\right)$ to refer to an argument of the form $\epsilon$ )

- $\mathcal{R}_{\mathcal{M}}$ is defined by $\mathcal{D}_{v}$.

The $V-M A F$ of an $a V A F(\mathcal{A}, \mathcal{R}, V, v a l, \mathrm{a})$ is defined as above, where $P=\{\mathrm{a}\}$.

Example 1 Figure 7a) shows the $V-M A F$ formulation of the $V A F$ :

$\mathcal{A}=\{a, b\}$

$\mathcal{R}=\{(a, b)\}$

$V=\{v 1, v 2\}$

$\operatorname{val}(a)=v 1, \operatorname{val}(b)=v 2$

$P=\left\{\mathrm{a}_{1}=\left\{\left(v_{1}, v_{2}\right)\right\}, \mathrm{a}_{2}=\left\{\left(v_{2}, v_{1}\right)\right\}\right\}$

We have two preferred extensions of the $V-M A F$ formulation, one for each audience:

$E 1=\left\{\left(>_{a 1}\right),\left(a_{v 1} P b_{v 2}\right),(j-a),(a \operatorname{def} b),(r-b)\right\}$

$E 2=\left\{\left(>_{a 2}\right),\left(b_{v 2} P a_{v 1}\right),(j-a),(j-b)\right\}$

$E 2$ is then the single preferred extension of the $V-M A F$ formulation of the $a V A F$ for audience $a 2$ shown in Figure $7 \mathrm{~b}$ ).

Theorem 5 Let $\Delta_{M}=\left(\mathcal{A}_{\mathcal{M}}, \mathcal{R}_{\mathcal{M}}, \mathcal{C}, \mathcal{L}_{\mathcal{M}}, \mathcal{D}_{v}\right)$ be the $V-M A F$ of an $a V A F \Delta=$ $(\mathcal{A}, \mathcal{R}, V, v a l, a)$. Then for $s \in\{$ complete, grounded, preferred, stable $\},(j-x) \in \mathcal{A}_{\mathcal{M}}$ is a credulously, respectively sceptically, justified argument of $\Delta_{M}$ under the $s$ semantics, iff $x \in \mathcal{A}$ is a credulously, respectively sceptically, justified argument of $\Delta$ under the $s$ semantics.

In [12], the arguments that appear in every preferred extension for every audience in a $V A F$ are referred to as objectively acceptable. The arguments that appear in at 


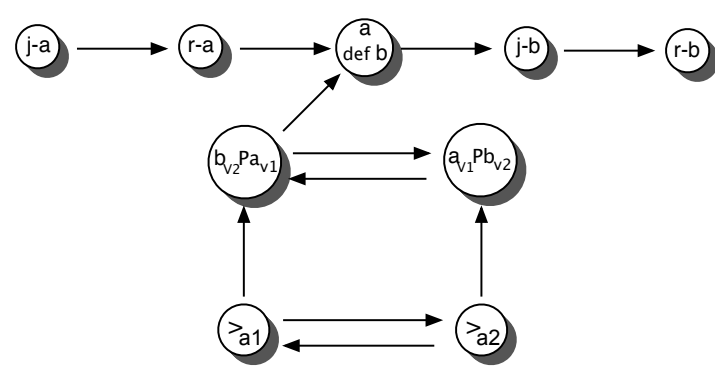

a)

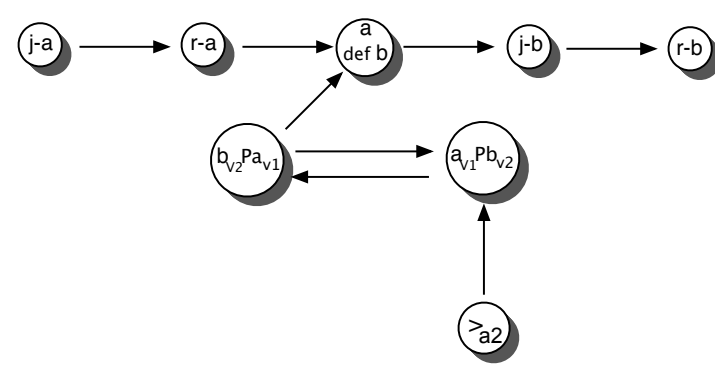

b)

Figure 7: A VAF (a)) and aVAF (b) formulated as metalevel frameworks

least one preferred extension for at least one audience in a $V A F$ are referred to as subjectively acceptable. These notions correspond to the sceptically, respectively credulously justified arguments (under the preferred semantics) of the $V A F$ 's metalevel formulation.

Definition 26 [Objective and Subjective Acceptance]

Given a $V A F \Delta=(\mathcal{A}, \mathcal{R}, V$, val, $P)$, and an argument $x \in \mathcal{A}$ :

- $x$ is objectively acceptable iff $\forall a \in P, x$ is a sceptically justified argument of the $a V A F(\mathcal{A}, \mathcal{R}, V, v a l, \mathrm{a})$ under the preferred semantics.

- $x$ is subjectively acceptable iff $\exists a \in P, x$ is a credulously justified argument of the $a V A F(\mathcal{A}, \mathcal{R}, V, v a l, \mathrm{a})$ under the preferred semantics.

Theorem 6 Let $\Delta_{M}=\left(\mathcal{A}_{\mathcal{M}}, \mathcal{R}_{\mathcal{M}}, \mathcal{C}, \mathcal{L}_{\mathcal{M}}, \mathcal{D}_{v}\right)$ be the $V-M A F$ of a $V A F \Delta=$ $(\mathcal{A}, \mathcal{R}, V, v a l, P)$. Then for any $x \in \mathcal{A},(j-x) \in \mathcal{A}_{\mathcal{M}}$ :

1. $x$ is an objectively acceptable argument of $\Delta$ iff $(j-x)$ is a sceptically justified argument of $\Delta_{M}$ under the preferred semantics.

2. $x$ is a subjectively acceptable argument of $\Delta$ iff $(j-x)$ is a credulously justified argument of $\Delta_{M}$ under the preferred semantics. 


\subsection{Formalising Extended Argumentation in Meta-level Argumen- tation Frameworks}

In both preference and value based argumentation, information assumed to be exogenous to the domain of argumentation based reasoning is used to undermine the success of attack as defeats. In $E A F \mathrm{~s}$, such information is part of the object level domain of argumentation, and in keeping with the abstract nature of Dung's approach no commitments are made to the nature of this information. Rather, the use of the information to undermine the success of attacks is abstractly characterised; by defining a new attack relation that originates from an argument, and that attacks an attack.

Intuitively, the metalevel characterisation of the object level reasoning in an $E A F$, extends that in a Dung $A F$ so that arguments of the form $(j-x)$ and arguments of the form $(q$ def $r)$ are attacked respectively by arguments of the form $(y$ def $x)$ and $(p$ def $(q \operatorname{def} r))$. Just as $(y \operatorname{def} x)$ is attacked by $(r-y)$ and $(r-y)$ is attacked by $(j-y)$, so $(p \operatorname{def}(q \operatorname{def} r))$ is attacked by $(r-p)$ and $(r-p)$ is attacked by $(j-p)$.

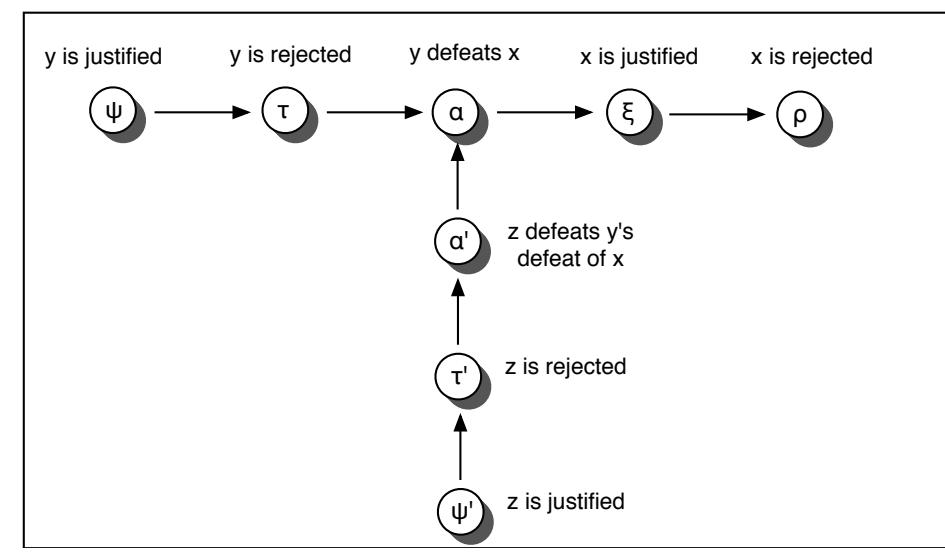

Figure 8: Meta-level formulation of an attack on an attack

Hence, in addition to MR1, MR2 and MR3 in Section 3.1:

MR1' The existence of an attack $(z,(y, x))$, constitutes a metalevel argument $\alpha^{\prime}$ claiming ' $z$ successfully attacks and so defeats $y$ 's defeat of $x$ '; hence $\alpha$ ' attacks $\alpha$ (see Figure 8). Any metalevel attack on $\alpha^{\prime}$, challenging $z$ 's defeat of $y$ 's defeat of $x$, reinstates $\alpha$, and characterises the object level reinstatement of $y$ 's attack on $x$.

MR2' If $z$ is rejected, then $z$ does not defeat $y$ 's defeat of $x$. Hence, $\alpha^{\prime}$ is attacked by $\tau^{\prime}$ claiming that ' $z$ is rejected'. Thus $\tau^{\prime}$ reinstates $\alpha$.

MR3' If $z$ is justified, then $z$ defeats $y$ 's defeat of $x$. Thus, $\psi^{\prime}$ claiming that ' $z$ is justified' attacks $\tau^{\prime}$ claiming ' $z$ is rejected', so reinstating $\alpha^{\prime}$.

Definition 27 [Extended Argumentation as a Meta-level Argumentation Framework] An $E-M A F$ is a tuple $\left(\mathcal{A}_{\mathcal{M}}, \mathcal{R}_{\mathcal{M}}, \mathcal{C}, \mathcal{L}_{\mathcal{M}}, \mathcal{D}_{e}\right)$, where:

$\mathcal{D}_{e}=\mathcal{D}_{d} \cup\{D 4:$ if $\mathcal{C}(\alpha)=\operatorname{defeat}(Z,(\operatorname{defeat}(Y, X))$ and $\mathcal{C}(\beta)=\operatorname{defeat}(Y, X)$ then $\left.(\alpha, \beta) \in \mathcal{R}_{\mathcal{M}}\right\}$. 
Definition 28 [Mapping EAFs to Metalevel Frameworks] An $E-M A F \Delta_{M}=\left(\mathcal{A}_{\mathcal{M}}\right.$, $\left.\mathcal{R}_{\mathcal{M}}, \mathcal{C}, \mathcal{L}_{\mathcal{M}}, \mathcal{D}_{e}\right)$ is said to be a metalevel formulation of the $E A F \Delta=(\mathcal{A}, \mathcal{R}, \mathcal{D})$ iff:

- $\lceil x\rceil$ is a constant in $\mathcal{L}_{\mathcal{M}}$ iff $x \in \mathcal{A}$

- $\mathcal{A}_{\mathcal{M}}$ is the union of the disjoint sets $\mathcal{A}_{\mathcal{M} 1}, \mathcal{A}_{\mathcal{M} 2}, \mathcal{A}_{\mathcal{M} 3}, \mathcal{A}_{\mathcal{M} 4}$, where $\mathcal{A}_{\mathcal{M} 1}$, $\mathcal{A}_{\mathcal{M} 2}$ and $\mathcal{A}_{\mathcal{M} 3}$ are defined as in Definition 19, and:

4. $\delta \in \mathcal{A}_{\mathcal{M}}, \mathcal{C}(\gamma)=\operatorname{defeat}(\lceil z\rceil,(\operatorname{defeat}(\lceil y\rceil,\lceil x\rceil))$ iff $(z,(y, x)) \in \mathcal{D}$

(from hereon, we write $(z D(y D x))$ to refer to an argument of the form $\delta$ ).

- $\mathcal{R}_{\mathcal{M}}$ is defined by $\mathcal{D}_{e}$.

Figure 9 shows the metalevel formulation of the weather example $E A F$ in Figure 2b).



Figure 9: Meta-level formulation of the $E A F$ in Figure 2b)

In general, a correspondence does not hold between the justified arguments of $E A F \mathrm{~s}$ and their metalevel formulations. Consider the $E A F \Delta$ in Figure 10, in which $a$ attacks $b$, and $b$ itself attacks the attack from $a^{6} . \Delta$ 's single preferred extension is $E=\{a, b\}$, since $E$ is conflict free according to Definition 13, $a$ is obviously acceptable w.r.t. $E$, and $b$ is acceptable w.r.t. $E$ since no argument $\operatorname{defeats}_{E} b$. However, there exist two preferred extensions of $\Delta$ 's metalevel formulation $\Delta_{M}$ :

$$
\{(j-a),(a \operatorname{def} b),(r-b)\} \text { and }\{(j-a),(j-b),(b D(a D b))\}
$$

Hence, $a$ and $b$ are sceptically justified arguments of $\Delta$, whereas only $(j-a)$ is a sceptically justified argument of $\Delta_{M}$.

\footnotetext{
${ }^{6}$ The example demonstrates the kind of self-reference exhibited by the liar paradox ("this sentence is false") in that $b$ is interpreted as asserting a conclusion about itself, viz. that " $b$ is preferred to $a$ "
} 


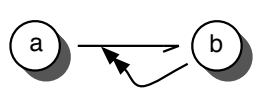

$\Delta$

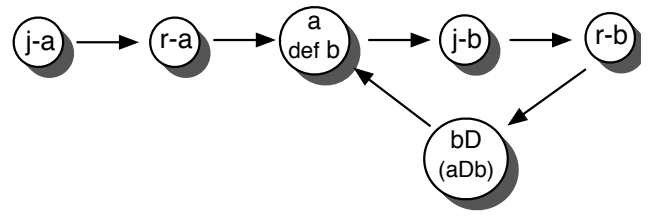

$\Delta_{\mathrm{M}}$

Figure 10: A non-hierarchical $E A F$ and its metalevel formulation $\Delta_{M}$

The key thing to note in this example is that $E A F$ s do not impose restrictions on the interactions between arguments. Hence, information applied to undermine the success of attacks as defeats between object level arguments, can itself be part of the object level domain of argumentation (in contrast with $P A F \mathrm{~s}$ and $V A F \mathrm{~s}$, in which such information is meta to the object level arguments). With reference to the above example, we now discuss how this 'mixing' of the meta and object level in EAFs reveals a distinction between the ontological status ascribed to $\mathcal{R}$ attacks in $E A F$ s and their metalevel formulation as arguments, and thus results in the correspondence not holding.

If one were to ascribe to attacks the same status as arguments, then one might justifiably consider $\{a, b\}$ and $\{a\}$ as distinct preferred extensions of the $E A F \Delta$, where the latter preferred extension implicitly contains the attack $a \rightarrow b$. However, $E A F$ s treat $\mathcal{R}$ attacks as second class citizens, and with some justification given that the existence of an attack is contingent on the existence of arguments but not vice versa (i.e., one can consider the existence of arguments independently of attacks, but not vice versa). In this view it is legitimate to identify $\{a, b\}$ as the set inclusion maximal admissible set of arguments.

In our metalevel formulation, one does not discriminate between metalevel arguments constituted by the existence of $\mathcal{R}$ attacks and arguments in $\mathcal{A}$; these attacks and arguments are effectively assumed to be on a par. This in turn means that unlike attacks at the object level, attacks formalised as metalevel arguments can indirectly reinstate themselves. This is illustrated in $\Delta_{M}$ in Figure 10 in which $(a$ def $b)$ is part of a 4 cycle, so that $(a$ def $b)$ reinstates $(r-b)$, which in turn reinstates $(a$ def $b)$ (hence we say $(a$ def $b)$ indirectly reinstates itself) in the preferred extension $\{(j-a),(a$ def $b),(r-b)\}$. Now notice that this implication of the ontologically distinct treatment of attacks at the object and metalevel would not manifest itself if we focussed on EAFs that maintain a strict separation between object level arguments and the arguments that attack attacks between object level arguments. If such a separation were imposed, then an argument of the form ( $a$ def $b$ ) would not be able to indirectly reinstate itself, and we would thus expect a correspondence to hold.

Indeed, [38] study a special class of hierarchical EAF in which the argumentation is 'stratified' into levels so that, intuitively, each level is a Dung framework $(\mathcal{A}, \mathcal{R})$ in which all $\mathcal{R}$ attacks are restricted to arguments within the framework. These $\mathcal{R}$ attacks are then attacked by $\mathcal{D}$ attacks that exclusively originate from arguments in the immediate metalevel ${ }^{7}$. It is interesting to note that the characteristic functions of hierarchical $E A F$ s are monotonic, so enabling characterisation of their grounded extensions as the least fixed point of their characteristic functions.

\footnotetext{
${ }^{7}$ Although intuitively this could be generalised to any, rather than just the immediate metalevel.
} 
Definition 29 [Hierarchical $\boldsymbol{E A F} \mathbf{s}$ ] $\Delta=(\mathcal{A}, \mathcal{R}, \mathcal{D})$ is a hierarchical EAF iff there exists a partition $\Delta_{H}=\left(\left(\left(\mathcal{A}_{1}, \mathcal{R}_{1}\right), \mathcal{D}_{1}\right), \ldots,\left(\left(\mathcal{A}_{j}, \mathcal{R}_{j}\right), \mathcal{D}_{j}\right), \ldots\right)$ such that:

- $\mathcal{A}=\bigcup_{i=1}^{\infty} \mathcal{A}_{i}, \mathcal{R}=\bigcup_{i=1}^{\infty} \mathcal{R}_{i}, \mathcal{D}=\bigcup_{i=1}^{\infty} \mathcal{D}_{i}$, and for $i=1 \ldots \infty,\left(\mathcal{A}_{i}, \mathcal{R}_{i}\right)$ is a Dung argumentation framework.

- $(C,(A, B)) \in \mathcal{D}_{i}$ implies $(A, B) \in \mathcal{R}_{i}, C \in \mathcal{A}_{i+1}$

$\Delta$ is a bounded hierarchical EAF iff its partition $\Delta_{H}$ is of the form $\left(\left(\left(\mathcal{A}_{1}, \mathcal{R}_{1}\right), \mathcal{D}_{1}\right)\right.$, $\left.\ldots,\left(\left(\mathcal{A}_{n}, \mathcal{R}_{n}\right), \mathcal{D}_{n}\right)\right)$, where $\mathcal{D}_{n}=\emptyset$

A correspondence can then be shown between bounded hierarchical EAFs and their metalevel formulations:

Theorem 7 Let $\Delta_{M}=\left(\mathcal{A}_{\mathcal{M}}, \mathcal{R}_{\mathcal{M}}, \mathcal{C}, \mathcal{L}_{\mathcal{M}}, \mathcal{D}_{e}\right)$ be the $E-M A F$ of a bounded hierarchical $E A F \Delta=(\mathcal{A}, \mathcal{R}, \mathcal{D})$. Then for $s \in\{$ complete, grounded, preferred, stable $\}$, $(j-x) \in \mathcal{A}_{\mathcal{M}}$ is a credulously, respectively sceptically, justified argument of $\Delta_{M}$ under the $s$ semantics, iff $x \in \mathcal{A}$ is a credulously, respectively sceptically, justified argument of $\Delta$ under the $s$ semantics.

The $E A F \triangle$ in Figure 8 is not hierarchical. However the weather example $E A F$ in Figure $2 \mathrm{~b}$ ) is bounded hierarchical. Its single preferred extension $\{e, d, b\}$ corresponds to the single preferred extension

$$
\begin{aligned}
& \{(j-e),(e D(c D d)),(j-d),(d \operatorname{def} c),(r-c),(d D(a D b)),(j-b),(b \\
& \operatorname{def} a),(r-a)\}
\end{aligned}
$$

of its metalevel formulation in Figure 10.

Although [38] discusses and illustrates requirements for $E A F$ s that do not conform to the hierarchical restriction, we observe that many applications of extended argumentation can be naturally accommodated under the hierarchical restriction; in particular application of extended argumentation to agent reasoning over beliefs, goals and actions [43, 37]. Also, reinterpreting the arguments based on preferences in PAFs and on value preferences in $V A F$ s produces hierarchical frameworks. Note that in Section 6 we comment further on the lack of correspondence between non-hierarchical $E A F \mathrm{~s}$ and their metalevel formulations.

\section{Applications of Metalevel Argumentation}

\subsection{Applying Results and Techniques for Dung Argumentation to Developments of Dung Argumentation}

In the previous section we described metalevel formulations of various object level developments of Dung's abstract argumentation theory, and showed that the justified arguments of the object level frameworks can be characterised in terms of the justified arguments of their metalevel formulations. These correspondences allow one to transition the full range of theoretical and practical results and techniques defined for Dung argumentation, to these various developments. For example, consider the use of labellings for characterising the extensions of a Dung framework, reviewed in Section 2.1.2. Algorithms for computing labellings, and therefore extensions, have also been proposed [23, 42, 51, 52]. Given the correspondences between object level and 
metalavel extensions, one can now make use of the labelling approach and algorithms at the metalevel in order to characterise and compute the object level extensions. For example, suppose $\Delta$ is an object level hierarchical $E A F$, and let $\Delta_{M}=\left(\mathcal{A}_{\mathcal{M}}, \mathcal{R}_{\mathcal{M}}\right.$, $\mathcal{C}, \mathcal{L}_{\mathcal{M}}, \mathcal{D}_{e}$ ) be its $E-M A F$ as defined in Definition 28. We can then apply labelling algorithms to compute the labellings and so extensions of the Dung framework $\left(\mathcal{A}_{\mathcal{M}}\right.$, $\mathcal{R}_{\mathcal{M}}$ ) under all of Dung's semantics. Given Theorem 7, we thus obtain the extensions of $\Delta^{8}$

Argument game proof theories for Dung frameworks have also been defined for establishing the justified status of a given argument $x$ under each of Dung's semantics $[24,28,42,53]$. Given the correspondences shown in the previous section, one can make use of these proof theories to establish the justified status of arguments of the form $(j-x)$ in order to establish the status of $x$ in the corresponding object level framework. In what follows, we describe games applied to our metalevel formulation of value based argumentation [12], and contrast the use of such games with the more complex games specifically developed for value based argumentation [11, 13].

\subsubsection{Applying Argument Game Proof Theories to Metalevel Argumentation Frameworks}

Argument games take the form of dialogues between a proponent and an opponent of a given argument. The proponent starts with an argument to be tested, after which each player must attack the other player's arguments with a counterargument of sufficient strength. The initial argument is provable if the proponent has a winning strategy, i.e., if he can make the opponent run out of moves however the opponent chooses to attack. Essentially the dialogue constructs an admissible set containing the desired argument. The precise rules of the argument game depend on the semantics which the proof theory is meant to capture. Games for demonstrating the justified status under credulous preferred and sceptical preferred semantics, for coherent frameworks ${ }^{9}$, are given in [53]. These games, which are Two Player Immediate Response (TPI) games, in which a player must address the argument last played, were refined and their properties explored in [28]. In the latter work three moves are used: COUNTER, BACKUP and RETRACT.

COUNTER can be made by either player, and involves playing an argument which attacks the last argument played. BACKUP is only employed by opponent, and RETRACT only by proponent. These two moves are different for the different players, and arise from the need to allow back-tracking, when there is no argument available to attack the argument last played. Firstly, BACKUP may be seen as opponent invoking a new line of attack within the same dispute tree. On the other hand, RETRACT represents the dispute being started again, this time, however, with the knowledge that some lines of defence are not available, i.e., those that would result in a known inadmissible set being constructed. In fact, as was shown in [28], if the argument is credulously preferred then a proponent employing 'best play' will never need to make a retraction: RETRACT is needed only to allow for strategic mistakes. Making moves can affect the arguments available for subsequent use. If an argument is played which attacks arguments as yet unplayed, the attacked arguments cease to be available for the proponent (in a sceptical

\footnotetext{
${ }^{8}$ Note that labellings have recently been defined directly on arbitrary $E A F$ s for only the admissible, preferred and stable semantics [39]. In this work algorithms for computing these extensions are not defined.

${ }^{9}$ Every preferred extension of a coherent framework is also stable, and since in general stable extensions are preferred, the stable and preferred extensions of a coherent framework coincide.
} 
game) or the opponent (in a credulous game). Similarly arguments attacking an argument played are not available to the player who played the latter attacked argument.

These games have been shown to be sound and complete ${ }^{10}$ and work very well for Dung frameworks, and attempts have been made to extend the games to accommodate preferences. For example, a game for value based frameworks was proposed in [11]. In that game an additional VALUE move is made available to both players. The VALUE move is used when there is no argument available to allow a COUNTER move, and allows the player to defend an argument by claiming an audience advocating that its value is preferred to its attacker. A record of such moves must be kept so as to block a VALUE move expressing an audience whose value preference contradicts a previous VALUE move's audience advocated preference. Although this game is effective for some frameworks, it is more complicated than the original TPI, in that it has this additional move, and it requires maintenance of additional structures to record the currently expressed value preferences. Additionally certain types of framework present problems.

We can illustrate these problems by reference to the $V A F$ in Figure 11a), in which $x 5$ is objectively acceptable (i.e. sceptically justified under the preferred semantics irrespective of the relative ordering of the values property and life). If proponent starts a game with $x 5$, which is then attacked by opponent's $x 6$, proponent cannot then COUNTER with $x 7$, since $x 7$ is attacked by an argument already moved by proponent, i.e., $x 5$ itself. Hence proponent uses the VALUE move to claim life > property. While this will succeed in establishing the acceptability of $x 5$, it should not have been necessary to express this audience since $x 5$ is acceptable irrespective of the audience.

Consider, however, that we can apply the standard TPI games to our metalevel formulations of VAFs. Given Theorem 5, we can then evaluate the justified status of an argument $(j-x)$ in the metalevel formulation, so evaluating the status of $x$ in the object level $V A F$. For example, we can play the standard sceptical TPI game on the metalevel formulation of the $V A F$ in Figure 11b) in which $\left(>_{a 1}\right)$ denotes the argument claiming audience $(\{($ property, life $)\})$ and $\left(>_{\mathrm{a} 2}\right)$ the argument claiming audience $(\{($ life, property $)\})$. Note that in this sceptical game opponent has only to show that he is not compelled to accept a proponent's proposed argument, and so opponent can play arguments already attacked by proponent arguments. Suppose that when opponent plays $(x 6$ def $x 5)$, proponent challenges with $\left(x 5_{L} P x 6_{P}\right)$ ('L' denotes life and ' $\mathrm{P}$ ' denotes property). The opponent can then continue along either of two lines of attack visualised in Figure 11c). In either case proponent cannot repeat $\left(>_{a} 2\right.$ ) given that it is already attacked by opponent. Hence, in either case, proponent is forced to RETRACT $\left(>_{a 1}\right)$ and its endorsed value preference $\left(x 5_{L} P x 6_{P}\right)$, and initiate a new line of defence, attacking $(x 6$ def $x 5)$ with $(r-x 6)$. The dispute continues as visualised in Figure 11d), eventually leading to proponent playing $(j-x 7)$ (note that $(j-x 7)$ is not directly attacked by proponent's $(j-x 5))$, opponent playing $(x 5$ def $x 7)$, and then proponent $\left(x 7_{P} P x 5_{L}\right)$. Now, according to the rules of [28]'s TPI game, opponent can play neither of $\left(x 5_{L} P x 7_{P}\right)$ or $\left(>_{a 2}\right)$, since both arguments are attacked by the argument $\left(>_{a 1}\right)$ that opponent has played in whichever of the dispute lines in Figure 11c) that has led to proponent's retraction. Proponent effectively demonstrates that the audience opponent commits to in order to undermine proponent's defence, also supports proponent's defence. Notice that if the proponent's first attempt at a defence had been to play $(r-x 6)$, this would have lead to either of the two opponent

\footnotetext{
${ }^{10}$ That is to say there is a winning strategy for argument $x$ in a game played under the rules for sceptical, respectively credulous preferred semantics iff $x$ is justified under the sceptical, respectively credulous preferred semantics.
} 


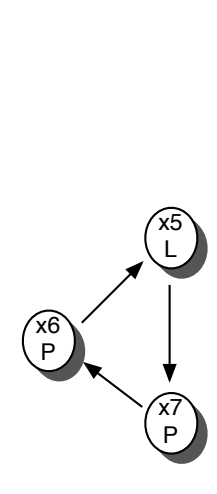

a)

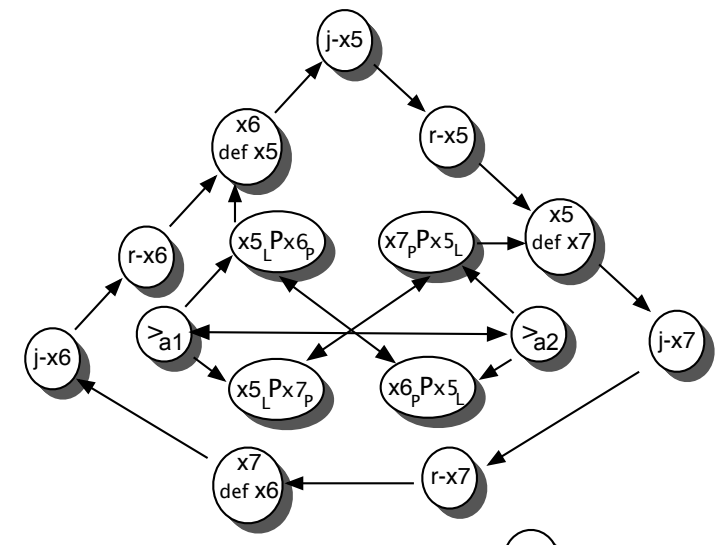

b)

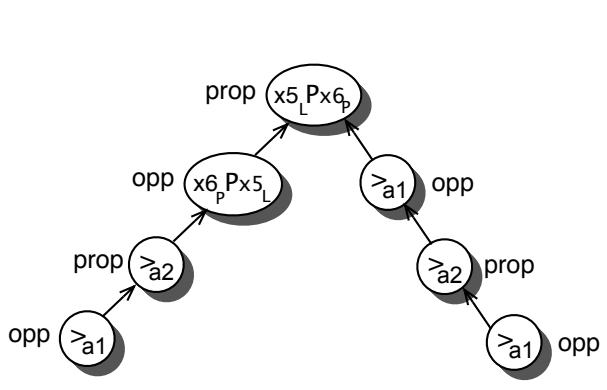

c)

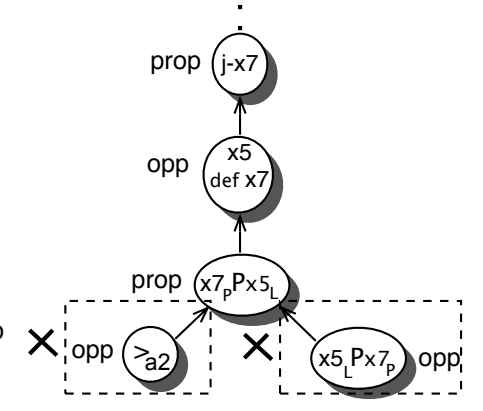

d)

Figure 11: a) a $V A F$ (L stands for life and $\mathrm{P}$ for property), and b) its metalevel formulation. c) shows two winning TPI dispute lines for opponent and d) a winning TPI dispute line for proponent in which opponent cannot play arguments attacked by the $\operatorname{argument}\left(>_{\mathrm{a} 1}\right.$ that is has played in both lines in $\left.\left.\mathrm{c}\right)\right)$

winning lines of attack in Figure 11d), in which the prohibited moves shown would have been allowed, and where the the right hand line of attack would now terminate by opponent playing $\left(>_{a 2}\right)$ in response to proponent's $\left(>_{a 1}\right)$. This would then similarly force proponent to RETRACT and then play $\left(x 5_{L} P x 6_{P}\right)$ as shown in Figure $\left.11 \mathrm{c}\right)$, and opponent would then be prohibited from playing either $\left(x 6_{P} P x 5_{L}\right)$ or $\left(>_{\mathrm{a} 1}\right)$. Hence, in either case proponent is able to establish the objective acceptability of $(j-x 5)$ (and so $x 5$ in the object level framework) without committing to any audience.

Another desirable feature of $V A F \mathrm{~s}$ is that rather than the audience being fixed in advance, it should emerge from the reasoning process itself. This view that value preferences are the product of reasoning rather than an input to it was expressed by Searle as follows:

This answer [that we can rank values in advance] while acceptable as far as it goes [as an ex post explanation], mistakenly implies that the preferences are given prior to practical reasoning, whereas, it seems to me, they are typically the product of practical reasoning. And since ordered preferences are typically products of practical reason, they cannot be treated as its 
universal presupposition. [50]

This issue is explored in [13], in which the set up is that given a $V A F$, the proponent wishes to defend a position in which the proponent wishes certain arguments to be accepted, certain other arguments to be rejected, and is indifferent to the inclusion or exclusion of the remaining arguments. [13] presents a game in which an audience for which the position is acceptable is determined, or it is shown that no such audience exists. The moves of the game are, however, rather complicated, and lack the clear intuitions of the TPI game moves. In the metalevel framework, however, the value preferences and audiences appear as part of the admissible set constructed during the course of a TPI dispute, and so we can play the TPI game to identify the constraints put upon the audience.

To illustrate, consider again the $V-M A F$ of Figure 11b), and suppose proponent wishes to accept $(j-x 7)$. Opponent challenges with $(x 5$ def $x 7)$. Examining the framework, we see that it is futile for proponent to play $(r-x 5)$, since this will ultimately require proponent to play $(r-x 7)$, which he has already attacked. Therefore proponent must play $\left(x 7_{P} P x 5_{L}\right)$, and then $\left(>_{\mathrm{a} 1}\right)$ in response to either of opponent's possible attacks. Since in this game proponent is only required to establish subjective acceptance (i.e., $(j-x 7)$ is credulously justified), opponent cannot now play arguments already attacked by proponent arguments. Specifically, opponent cannot play $\left(>_{a 2}\right)$, and so the game terminates, with the audience established as $\left(>_{a 1}\right)$ ordering property over life.

We conclude by observing that a number of efficiency gains can be obtained when applying argument games to metalevel frameworks in general. For example, one could allow a player to play more than one argument in a single move. In particular a player could move an argument of the form $(x \operatorname{def} y)$, followed by an argument of the form $(j-x)$, given that the player's counterpart will always be able to play $(r-x)$ in response to $(x \operatorname{def} y)$, which in turn can always be countered by $(j-x)$. If these two moves were played together the counterpart would then have the choice of attacking either $(x$ def $y)$ or $(j-x)$. Changes of this sort would eliminate some unnecessary rounds, but not otherwise impact on the game.

We can see then that use of standard TPI games played on a meta level framework improves greatly on the games played directly on VAFs. Unlike the game of [11], players are not obliged to make unnecessary commitments to audiences, and although the game of [13] is sound and complete, the game played on the metalevel framework is simpler since the game in [13] requires conditions on moves to ensure that incompatible value preferences are not expressed. Here, because value preferences and audiences are arguments in the framework, these considerations are handled uniformly through the attack mechanism, so that illegitimate arguments are clearly seen as not available because attacked by an argument to which the player is already committed. Further, the required preferences and the audiences themselves appear in the admissible set constructed by the dialogue, rather than being separately recovered from the history of the dialogue as in [13]. These improvements stem from the ability to use the clean framework provided by abstract argumentation to accommodate the preference considerations that require external mechanisms to express preferences at the object level.

Finally, notice that we can also apply TPI games, or other games defined for Dung frameworks [42], to our metalevel formulations of extended argumentation. We will return to this topic in our discussion of future work in Section 6. 


\subsection{Extending and Integrating Abstract Argumentation in Met- alevel Frameworks}

In the spirit of Dung's original theory, $M A F$ s adopt a level of abstraction that makes limited commitments to the instantiating logics. Thus metalevel arguments can be built from statements about the existence of arguments, preferences, attacks etc. in different object level frameworks, which in turn may be constructed from different underlying logics. This not only allows for integration and further extension of the various developments of abstract argumentation, but also provides principled means for instantiation by, and integration of arguments constructed from different theories in different underlying logics, where one theory may encode metalevel reasoning about the arguments defined by another theory .

\subsubsection{Extending Abstract Argumentation in Metalevel Frameworks}

Recall that in VAFs audiences serve as oracles in that it is not possible to debate the merits of belonging to one audience rather than another. At the metalevel, however, the audiences are arguments within the framework, and as such are open to attack like any other argument, allowing one to advance arguments for and against particular audiences. Consider Definition 24's metalevel formulation of value based argumentation. Given an object level $V A F$ we can construct the arguments and attacks instantiating a $V-M A F$, and additionally include in the $V-M A F$ metalevel arguments that refer to object level arguments and attacks defined by argumentation-based reasoning about what the audience should be.

One possible source of such arguments may be moral principles. In a debate on fox hunting, for example, one might find an argument along the lines of fox hunting is enjoyed by many people in conflict with an argument such as fox hunting causes animal suffering. Resolving this conflict requires choosing between the audience preferring the value of human enjoyment to the value of animal welfare, and the audience endorsing the contrary preference. Opponents of hunting could now appeal to some moral standards, claiming that it is not a legitimate choice to prefer human enjoyment to animal welfare, using an argument such as no rational person could promote enjoyment at the expense of animal welfare. Such an argument would attack any audience endorsing the preference for human enjoyment. In turn this argument could be subject to attack, so that the debate shifts to what preferences are legitimate.

This kind of argumentation is particularly common in the legal domain, especially when considering common law and the role of precedent cases. Often a case will turn on how the court chooses to resolve a conflict between possible purposes that the law can serve. Consider the well known property law case of Pierson v Post that has been the subject of much discussion since its introduction into AI and Law [25]. In this case there is a conflict between the value of encouraging a socially useful activity, and the need to have clear law to minimise disputes. While the minority opinion in Pierson favoured the first value, the majority preferred clear law to social utility. In subsequent cases where this choice is presented, Pierson can be cited as an argument against adopting a preference for social utility.

This additional expressiveness is important for representing such domains. Whereas object level frameworks, such as that produced for Pierson and related cases in [16] could identify the choices confronting the courts, they could capture neither the choice actually made, nor the rationale for the choice, both of which are essential for a proper representation of precedential reasoning. For a recent representation of case law which 
uses metalevel frameworks to allow such argumentation, see [15].

Consider also that our definition of $E-M A F$ s (Definition 27) admits (given the BNF specification of $\mathcal{L}_{\mathcal{M}}$ in Definition 17) arguments with claims of the form defeat $\left(Z_{n}\right.$, defeat $\left(Z_{n-1}\right.$, defeat $\left.\left.\left(Z_{n-2}, \ldots\right)\right)\right)$, where each such argument attacks an argument with claim defeat $\left(Z_{n-1}\right.$, defeat $\left.\left(Z_{n-2}, \ldots\right)\right)$. That is to say we can model recursive attacks on attacks on attacks on ... . etc (see Figure 12). Indeed, such a metalevel formulation might even provide a basis for defining an object level framework extending $E A F$ s to accommodate such recursive attacks, in the sense that one might verify the correctness of such an object level formalisation by showing a correspondence with the metalevel formulation. Recently, [8] have proposed just such an object level formalisation of recursive attacks, and we will discuss this work in Section $5{ }^{11}$.

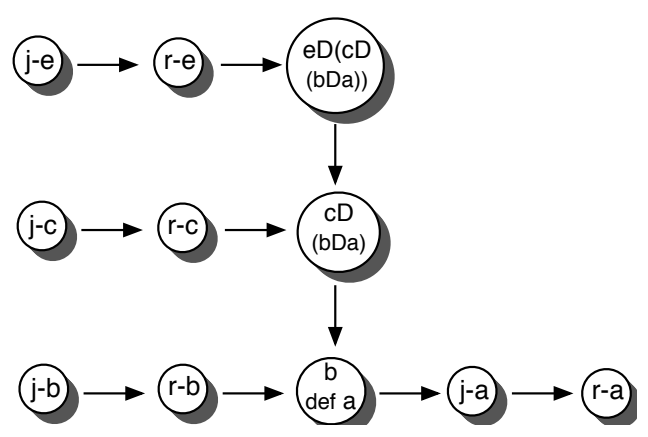

Figure 12: An $E-M A F$ with metalevel formulations of recursive attacks on attacks.

\subsubsection{Integrating Abstract Argumentation in Metalevel Frameworks}

A number of works (e.g., $[33,36])$ have described requirements for extending value based argumentation so that criteria other than the audience based ranking of values can be used to undermine the success of attacks. For example, consider that two arguments $a$ and $b$ symmetrically attack, where $a$ and $b$ promote the same value. One may then wish to arbitrate between $a$ and $b$ based on other criteria, such as the relative trustworthiness of the distinct advocates (or sources) of each argument, or the degree to which each argument promotes a value. We can formalise a metalevel integration of value and preference argumentation by straightforwardly combining the $P-M A F \mathrm{~s}$ and $V-M A F$ s of Sections 3.4 and 3.5 , by adding an additional constraint specifying attacks between contrary pairwise value preferences and strict preferences given by the preference relation.

Definition 30 [Integrating Value and Preference based Argumentation in a Metalevel Argumentation Framework]

A $V P-M A F$ is a tuple $\left(\mathcal{A}_{\mathcal{M}}, \mathcal{R}_{\mathcal{M}}, \mathcal{C}, \mathcal{L}_{\mathcal{M}}, \mathcal{D}_{v p}\right)$, where $\mathcal{D}_{v p}=\mathcal{D}_{v} \cup \mathcal{D}_{p} \cup$

$$
\begin{aligned}
& \left\{D^{\prime}: \text { if } \mathcal{C}(\alpha)=\text { val_pref }\left(\operatorname{val}\left(Y, V^{\prime}\right), \operatorname{val}(X, V)\right) \text { and } \mathcal{C}(\beta)=s_{-} \text {preferred }(X, Y)\right. \\
& \text { then } \left.(\beta, \alpha),(\alpha, \beta) \in \mathcal{R}_{\mathcal{M}}\right\} .
\end{aligned}
$$

\footnotetext{
${ }^{11}$ Note that the need for recursive attacks would also need to be carefully motivated. In the case of EAFs, attacks on attacks readily admit interpretation in terms of applying preferences, as substantiated in [38] by provision of $E A F$ semantics for [48]'s logic programming with defeasible priorities
} 
Example 2 Consider the $a V A F$ in Figure 13 where the single audience a 1 orders value $v 1$ over $v 2$. Consider also a separately defined preference ordering yielding the strict preferences $c \gg_{\mathcal{P}} b, a \gg_{\mathcal{P}} b$, where the latter strict preference resolves the choice between the symmetrically attacking $a$ and $b$, each of which promote the same value. Notice that there are two preferred extensions of the $V P-M A F$; the one containing the shaded arguments as shown in Figure 13b), and the second containing the same arguments except that $b_{v 1} P c_{v 2}$ replaces $(c$ def $b)$ and $(c P b)$. Both preferred extensions contain the sceptically justified arguments $(j-a)$ and $(j-c)$.

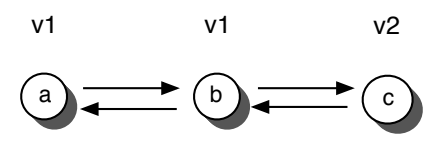

a)

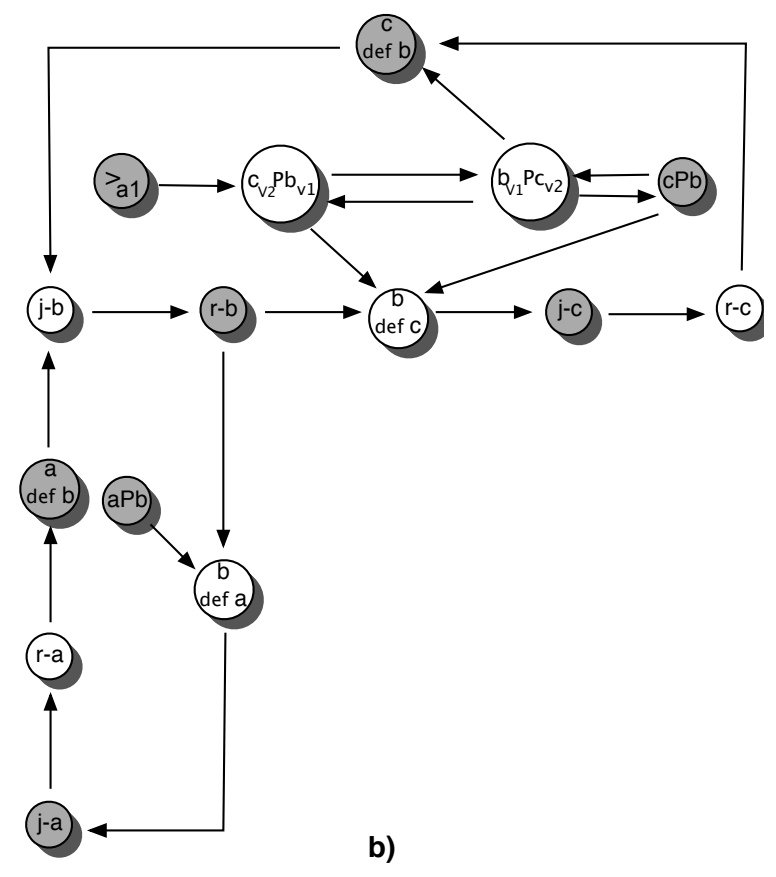

Figure 13: A $V A F$ (a) and preference ordering formalised as a $V P-M A F$ (b) in which the arguments in one of its two extensions are shaded

In the following example we illustrate the idea of metalevel integration of preference and value based arguments by referring to object level arguments for actions, value preferences, audiences and preferences constructed from different underlying logics and theories.

Example 3 Figure 14 shows the $V P-M A F\left(\mathcal{A}_{\mathcal{M}}, \mathcal{R}_{\mathcal{M}}, \mathcal{C}, \mathcal{L}_{\mathcal{M}}, \mathcal{D}_{v p}\right)$ illustrating argumentation-based reasoning about action. The $V P-M A F$ is instantiated as fol- 
lows:

- Arguments $(j-a 1),(j-a 2),(j-a 3) \in \mathcal{A}_{\mathcal{M}}$ are metalevel arguments claiming that object level arguments about action are justified, where these object level arguments are constructed in a BDI logic as described in [7]. $a 1$ and $a 2$ are arguments for the medical actions 'give aspirin' and 'give chlopidogrel' respectively. These arguments relate the current beliefs that warrant (are preconditions for) the actions bringing about states of affairs that realise a desired goal and so appeal to a value. $a 1$ and $a 2$ attack each other since they represent alternative courses of medical action for realising a given treatment goal. $a 3$ states that chlopidogrel is prohibitively expensive and so asymmetrically attacks $a 2$. Given $a 1, a 2$ and $a 3$, Figure 14 shows the metalevel arguments $(j-a 1)$, $(j-a 2),(j-a 3),(r-a 1),(r-a 2),(r-a 3),(a 1$ def $a 2),(a 2 \operatorname{def} a 1)$ and $(a 3$ def a2) related by metalevel attacks.

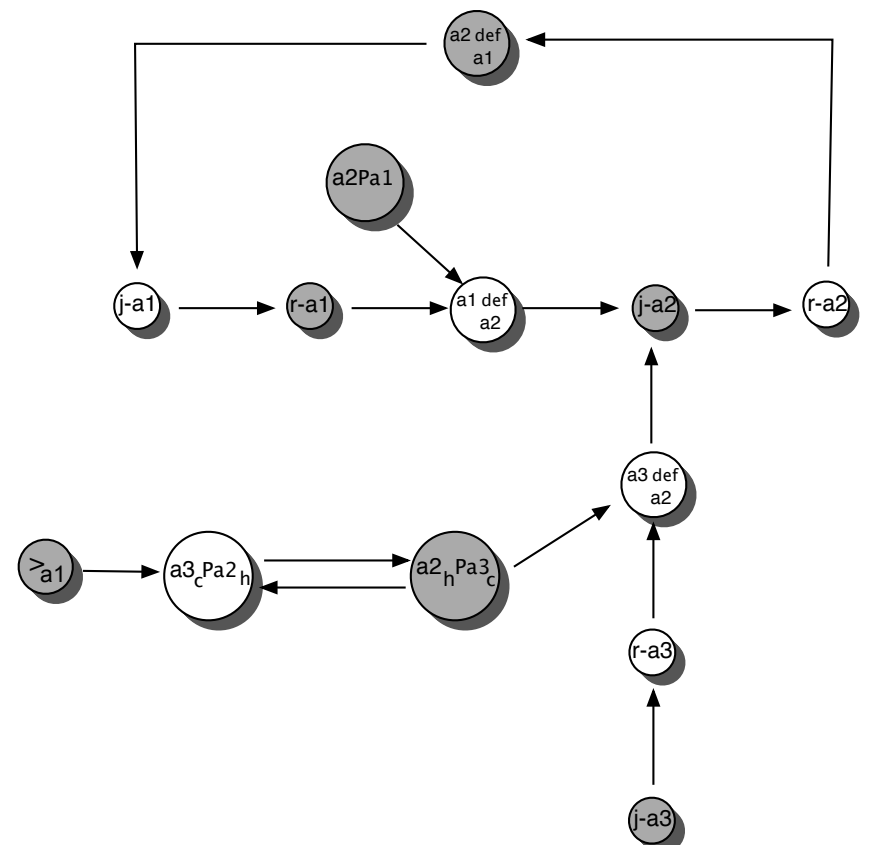

Figure 14: $V P-M A F$ for reasoning about medical actions

- In [36], construction of first order arguments that refer to the valuations of object level arguments is described. Specifically, [36] describes arguments built from first order theories consisting of facts that assign values to constants naming the above object level arguments for action, and orderings on values:

$$
\operatorname{val}(a 1, \text { health }), \operatorname{val}(a 2, \text { health }), \operatorname{val}(a 3, \text { cost }) \text { and }>(\mathrm{a} 1, \text { health, cost })
$$

Based on these first order arguments we construct the metalevel value preference and audience arguments:

$$
\left(\left(a 2_{\text {health }} \text { Pa } 3_{\text {cost }}\right)\right),\left(\left(a 3_{\text {cost }} P a 2_{\text {health }}\right)\right) \text { and }\left(>_{\mathrm{a} 1}\right)\left(\mathcal{C}\left(\left(>_{\mathrm{a} 1}\right)\right)=\{(\text { health, cost })\}\right) .
$$

- Finally, [36] also describes first order arguments constructed from first order theories describing clinical trial valuations of the relative efficacy of drugs. Here, the existence 
of a clinical trial argument in [36] concluding that chlopidogrel is more efficacious than aspirin at preventing blood clotting, is used to construct the metalevel argument $(a 2 P a 1)$ (i.e., $\mathcal{C}((a 2 P a 1))=$ s_preferred $(\lceil a 2\rceil,\lceil a 1\rceil))$.

The single preferred extension of the $V P-M A F$ contains the argument $(j-a 2)$ claiming that the argument for chlopidogrel is justified. It is more efficacious than aspirin, and the increased cost is discounted given that the value of health is deemed more important than cost (although in practice it is often the case endorsement of the contrary preference is argued for on utilitarian grounds).

\section{Related Work}

This paper builds on and substantially extends previous work of ours [41] in which bounded hierarchical $E A F$ s are rewritten as Dung frameworks. In [41] we 'expand' $\mathcal{R}$ attacks $x \rightarrow y$ in an $E A F$, to obtain attacks $x \rightarrow \bar{x} \rightarrow \overrightarrow{x y} \rightarrow y$. A $\mathcal{D}$ attack $(z,(x, y))$ is then rewritten as an attack $z \rightarrow \overrightarrow{x y}$ in the rewrite. We then show how one can formalise and extend value based argumentation in a hierarchical $E A F$, which is then rewritten as a Dung framework. The rewrites presented in [41] have inspired recent works by other authors [8,20]. Of particular interest is [8]'s extension of EAFs to accommodate recursive attacks on attacks, and their rewrite as Dung frameworks. It is instructive to examine how [8] accommodate attacks on attacks and formalise these as an object level Dung framework. Consider arguments $a, b, c, d$, and attacks $(a, b)$, $(c, d)$ and $(b,(c, d))$ ([8] also allow attacks on attacks on attacks etc.). The notion of direct and indirect defeats from attacks to arguments and attacks is defined, so that for the given example:

\section{$(a, b)$ directly defeats $b,(c, d)$ directly defeats $d,(b,(c, d))$ directly defeats $(c, d)$ and $(a, b)$ indirectly defeats $(b,(c, d))$}

Based on these notions of defeat, notions of conflict free, acceptability and admissible and preferred extensions are defined. A Dung argumentation framework rewrite is also defined. Figure 15a) shows [8]'s rewrite for the above example, and by way of comparison the metalevel $E-M A F$ formulation is also shown in Figure 15b). Intuitively, [8] effectively model attacks as arguments, in a manner similar to our metalevel formulation. Given that [8]'s motivation is to obtain a rewrite rather than formalise metalevel argumentation, the rewrite does not include arguments corresponding to metalevel arguments of the form $(r-x)$. Intuitively, however, a correspondence obtains between [8]'s rewrite and our metalevel formulation, since an argument $(r-b)$ will be acceptable iff ( $a$ def $b)$ is acceptable, so that one can formulate an attack directly from $(a$ def $b)$ to $(b D(c D d))$. These observations suggest therefore that the metalevel formulation of recursive attacks described in Section 4.2.1 can be viewed as a metalevel formulation of [8]'s object level formalisation of recursive attacks.

Finally, we mention works which formalise logics that explicitly refer to arguments and their relations and properties $[19,35,55]$. In particular, [55] also advocate the view that

"rational argumentation also involves putting forward arguments about arguments, and it is in this sense that they are meta-logical. For example, a statement that serves as a justification of an argument is a statement about an argument: the argument for which the justification serves must itself be referred to in the justification." 


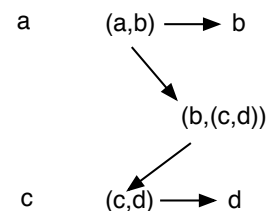

a)

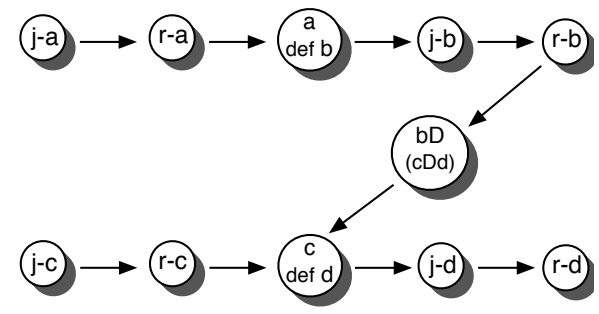

b)

Figure 15: Comparing rewrite in [8] (a) and the metalevel formulation of an $E A F$ (b)

Motivated by the claim that "argumentation and formal dialogue is necessarily a metalogical process", [55] fomalise metalogics in which one can reason about what constitutes an argument in the object level, attack and defeat relations between these arguments, their properties (e.g. the values they promote), and their status in the object level.

While [55] (and [19, 35]) provide meta-logics for reasoning about arguments, and argument construction based on these meta-logical statements, they do not relate these to argumentation frameworks, and so the argumentation at the metalevel is not considered using this single powerful abstraction. Indeed, one can see how these meta-logics can be used to define arguments, attacks and metalevel claims for instantiating the metalevel argumentation frameworks described in this paper.

\section{Conclusions}

We conclude with a summary of this paper's contributions and discuss directions for future work.

In this paper we defined a specific language in which arguments make claims about object level frameworks, thus identifying Metalevel Argumentation Frameworks in which the arguments are constructed from statements asserting the existence of object level arguments and their purported membership of admissible extensions, as well as statements asserting the existence of object level attacks, preference orderings on arguments, pairwise value preferences and audiences. The constraints on the metalevel attack relations, based on the claims about the object level frameworks, characterise the object level reasoning applied to evaluate the justified arguments, in terms of Dung's acceptability calculus. We have shown correspondences between the object level frameworks and their metalevel formulations; correspondences that not only yield a number of practical benefits, but also support a rhetorical aim of this paper to support the view that Dung's acceptability calculus identifies general and widely applicable principles of commonsense reasoning. We have shown how collective attacks, preference based, value based, and hierarchical extended argumentation can all be formulated as instances of Dung argumentation. The lack of correspondence with non-hierarhical $E A F$ s reflects differing intuitions about the ontological status of attack in [38]. Future work will look to more precisely identify when the correspondence does hold, given that the hierarchical restriction is overly prescriptive; intuitively, a correspondence should hold for any $E A F$ s whose metalevel formulations do not include attacks that directly, or indirectly, reinstate themselves. As mentioned in Section 4.2.1, 
future work will also investigate metalevel formulations of recursive attacks on attacks, and in particular investigate correspondences with the recursive attacks formalised in [8]. We will also investigate metalevel formulations of Dung frameworks augmented with support relations [3, 45]. For the moment, we observe that if $x$ supports $y$, then an attack on $x$ propagates to $y$. At the metalevel this would be formalised in terms of a metalevel attack from $(r-x)$ to $(j-y)$, so that if the argument $(r-x)$ claiming ' $x$ is rejected' is in an admissible extension $E$ (and is therefore reinstated by some $(z$ def $x) \in E)$, then it cannot be that $(j-y) \in E$.

In Section 4.1 we discussed how the correspondences shown in Section 3 allow one to transition the full range of theoretical and practical results and techniques for Dung argumentaton, to developments of Dung argumentation. We illustrated by showing how standard argument game proof theories for Dung frameworks can be be applied to value based argumentation frameworks, greatly improving on games specifically developed for the value based frameworks. Future work will further develop argument game proof theories for metalevel frameworks. In particular, the games will be applied to metalevel formulations of hierarchical extended argumentation, and we will investigate relationships with a game based proof theory recently defined for $E A F$ s [39] for the preferred credulous semantics. As discussed in Section 4.1, a key focus will also be on improving the efficiency of games for metalevel frameworks. Future work will also address application of other developments of Dung argumentation to metalevel frameworks, including labellings and labelling algorithms for computing metalevel extensions.

Argument game proof theories for Dung frameworks have informed formalisation of argumentation-based dialogues where, for example, one agent seeks to persuade another to adopt a belief it does not already hold to be true [47], or when agents deliberate about what actions to execute [32], or negotiate over resources [4]. Another direction for future work will be to formalise similar such dialogues for metalevel frameworks, allowing, for example, agents to debate value preferences in value based deliberation over actions, and preferences in negotiation.

In Section 4.2 we described how $M A F$ s can be instantiated by arguments built from statements about object level frameworks, thus facilitating extensions to, and integrations of various forms of abstract argumentation. We described how argumentation over audiences can be incorporated in metalevel formulations of $V A F \mathrm{~s}$, and how recursive attacks on attacks can be formulated in $E-M A F \mathrm{~s}$, so providing semantic guidelines for formalisation of object level Dung frameworks extended with recursive attacks. We also described how arguments for preference orderings and value preferences, built from different underlying theories encoded in different logics, can be integrated in metalevel integrations of value and preference based argumentation. These examples by no means exhaust the possible extensions and integrations, and there is much scope for future work in these areas. For example, one might look to integrate preferences with collective attacks, or model the strength of attacks [10] in terms of weights assigned to metalevel arguments of the form $(x$ def $y)$, where an attack's weight may need to exceed a certain threshold (as recently described in [29]), and the failure to do so may be modelled as a metalevel attack on $(x \operatorname{def} y)$.

\section{Appendix}

The following lemmas are used for the proofs of the main results in this paper. We first define the expansion of DungC and Dung frameworks, where, intuitively an expansion 
is obtained by some substituting some subset of the attacks $(X, y)$ in $\mathcal{R}$ (where in the case of a Dung framework $X$ is a single argument rather than a set of arguments) as shown in Figure 16.

Definition 31 [Expansion of a framework] Let $\Delta=(\mathcal{A}, \mathcal{R})$ be a DungC framework. Then $\Delta^{\prime}=\left(\mathcal{A}^{\prime}, \mathcal{R}^{\prime}\right)$ is said to be an expansion of $\Delta$ iff:

- $\mathcal{R}^{\prime}$ is any set of attacks $\left(\mathcal{R}^{*} \subseteq \mathcal{R}\right) \cup\left\{\operatorname{expand}((X, y)) \mid(X, y) \in\left(\mathcal{R}-\mathcal{R}^{*}\right)\right\}$ where expand $((X, y))=\{(\{x\}, \bar{x}),(\{\bar{x}\}, \overrightarrow{X y}) \mid x \in X\} \cup(\{\overrightarrow{X y}\}, y)\}$

- $\left.\mathcal{A}^{\prime}=\mathcal{A} \cup\left\{\bar{x}, \overrightarrow{X y} \mid(\{\bar{x}\}, \overrightarrow{X y}) \in \mathcal{R}^{\prime}\right)\right\}$

Let $\Delta=(\mathcal{A}, \mathcal{R})$ be a Dung framework. Then $\Delta^{\prime}=\left(\mathcal{A}^{\prime}, \mathcal{R}^{\prime}\right)$ is said to be an expansion of $\Delta$ iff $\Delta^{\prime}$ is the expansion of the DungC framework $\left(\mathcal{A}, \mathcal{R}^{s}\right)$ where $\mathcal{R}^{s}=$ $\{(\{x\}, y) \mid(x, y) \in \mathcal{R}\}$.

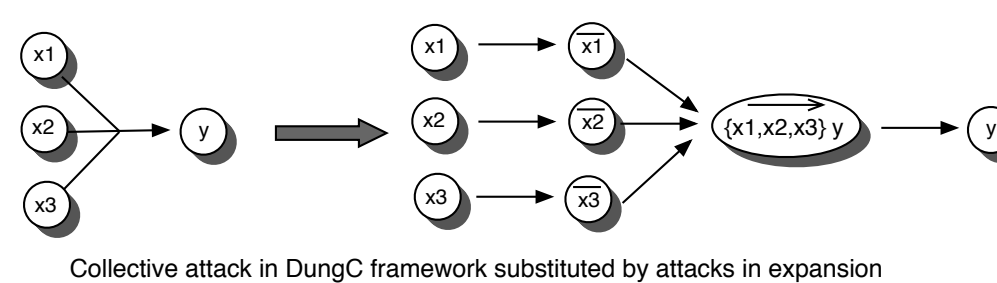

Collective attack in DungC framework substituted by attacks in expansion

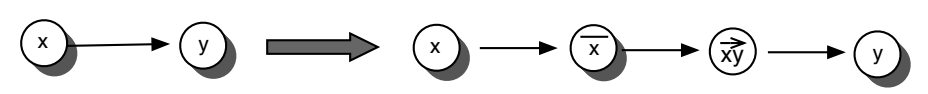

Attack in Dung framework substituted by attacks in expansion

Figure 16: Expansions of DungC collective attacks and Dung attacks

Lemmas 1 and 2 prove an iff correspondence between admissible extensions of DungC frameworks and their expansions.

Lemma 1 Let $\left(\mathcal{A}^{\prime}, \mathcal{R}^{\prime}\right)$ be an expansion of the DungC framework $(\mathcal{A}, \mathcal{R})$. Let $E$ be an admissible extension of $(\mathcal{A}, \mathcal{R})$. Then $E^{\prime}$ is an admissible extension of $\left(\mathcal{A}^{\prime}, \mathcal{R}^{\prime}\right)$ such that:

1. $E^{\prime}=E \cup\left\{\overrightarrow{X y} \mid X \subseteq E,(X, y) \in \mathcal{R}, \operatorname{expand}((X, y)) \subseteq \mathcal{R}^{\prime}\right\} \cup\{\bar{y} \mid X \subseteq$ $\left.E,(X, y) \in \mathcal{R}, \bar{y} \in \overline{\mathcal{A}^{\prime}}\right\}$

2. $\forall \alpha \in \mathcal{A}$, if $\alpha$ is acceptable w.r.t. $E$ then $\alpha$ is acceptable w.r.t. $E^{\prime}$

Proof: Let $\alpha \in \mathcal{A}$ be acceptable w.r.t. $E$. If $\neg \exists \Omega \subseteq \mathcal{A}$ s.t. $\Omega \mathcal{R} \alpha$, then $\alpha$ is not $\mathcal{R}^{\prime}$ attacked, and so $\alpha$ is acceptable w.r.t. $E^{\prime}$.

- Suppose $\exists \Omega \subseteq \mathcal{A}$ s.t. $\Omega \mathcal{R} \alpha$. By admissibility of $E, \exists \Gamma \subseteq E$ s.t. $\Gamma \mathcal{R} \delta, \delta \in \Omega$. Suppose $\Omega \mathcal{R}^{\prime} \alpha$ and it is not the case that $\Gamma \subseteq E^{\prime}, \Gamma \mathcal{R}^{\prime} \delta$. Then, $\Gamma \mathcal{R} \delta$ is some $\mathcal{R}$ attack $(X, y)$ such that expand $((X, y)) \subseteq \mathcal{R}^{\prime}$. By definition of $E^{\prime}, \overrightarrow{X y} \in E^{\prime}$, and since $\{\overrightarrow{X y}\} \mathcal{R}^{\prime} y, \alpha$ is acceptable w.r.t. $E^{\prime}$. 
- Suppose $\exists \Omega \subseteq \mathcal{A}^{\prime}$ s.t. $\Omega \mathcal{R}^{\prime} \alpha$, and it is not the case that $\Omega \mathcal{R} \alpha$. Then $\Omega$ is some $\overrightarrow{Y z}, \alpha$ some $z$, where $\forall y \in Y,\{y\} \mathcal{R}^{\prime} \bar{y},\{\bar{y}\} \mathcal{R}^{\prime} \overrightarrow{Y x}$. Since $\overrightarrow{Y z}$ is obtained by expanding $Y \mathcal{R} z$, and by admissibility of $E \exists X \subseteq E$ s.t. $X \mathcal{R} y^{\prime}$ for some $y^{\prime} \in Y$, then by definition of $E^{\prime}, \bar{y}^{\prime} \in E^{\prime}$, and so $\alpha(=z)$ is acceptable w.r.t $E^{\prime}$.

To show $E^{\prime}$ is admissible it remains to show that arguments $\overrightarrow{X y}, \bar{y} \in E^{\prime}$ are acceptable w.r.t. $E^{\prime}$. If $\overrightarrow{X y} \in E^{\prime}$ then $\forall x \in X,\{\bar{x}\} \mathcal{R}^{\prime} \overrightarrow{X y}$ and $\{x\} \mathcal{R}^{\prime} \bar{x}$, and since $X \subseteq E$ and so $X \subseteq E^{\prime}$, then $\overrightarrow{X y}$ is acceptable w.r.t. $E^{\prime}$. If $\bar{y} \in E^{\prime}$ then $(y, \bar{y}) \in \mathcal{R}^{\prime},(X, y) \in \mathcal{R}$ and $X \subseteq E$ hence $X \subseteq E^{\prime}$. If expand $((X, y)) \nsubseteq \mathcal{R}^{\prime}$ then $X \mathcal{R}^{\prime} y$, and so $\bar{y}$ is acceptable w.r.t. $E^{\prime}$. If expand $((X, y)) \subseteq \mathcal{R}^{\prime}$, then $\overrightarrow{X y} \in E^{\prime}$, where $\overrightarrow{X y} \mathcal{R}^{\prime} y$, hence $\bar{y}$ is acceptable w.r.t. $E^{\prime}$.

Lemma 2 Let $\left(\mathcal{A}^{\prime}, \mathcal{R}^{\prime}\right)$ be an expansion of the DungC framework $(\mathcal{A}, \mathcal{R})$. Let $E^{\prime}$ be an admissible extension of $\left(\mathcal{A}^{\prime}, \mathcal{R}^{\prime}\right)$. Then $E=\left(E^{\prime} \cap \mathcal{A}\right)$ is an admissible extension of $(\mathcal{A}, \mathcal{R})$ such that:

- $\overrightarrow{X y} \in E^{\prime}$ implies $X \subseteq E,(X, y) \in \mathcal{R}$, and $\bar{y} \in E^{\prime}$ implies $\exists X, X \subseteq E$, $(X, y) \in \mathcal{R}$

- $\forall \alpha \in \mathcal{A}, \alpha$ is acceptable w.r.t. $E^{\prime}$ implies $\alpha$ is acceptable w.r.t. $E$

Proof: Let $\alpha \in \mathcal{A}$ be acceptable w.r.t. $E^{\prime}$. If $\neg \exists \Omega \subseteq \mathcal{A}^{\prime}$ s.t. $\Omega \mathcal{R}^{\prime} \alpha$, then $\alpha$ is not $\mathcal{R}$ attacked and so $\alpha$ is acceptable w.r.t. $E$.

- Suppose $\exists \Omega \subseteq \mathcal{A}^{\prime}$ s.t. $\Omega \mathcal{R}^{\prime} \alpha$. By admissibility of $E^{\prime}, \exists \Gamma \subseteq E^{\prime}$ s.t. $\Gamma \mathcal{R}^{\prime} \delta, \delta \in \Omega$. Suppose $\Omega \mathcal{R} \alpha$ and it is not the case that $\Gamma \subseteq E, \Gamma \mathcal{R} \delta$. Then $\Gamma$ is of the form $\{\overrightarrow{X y}\}$ (and so $(X, y) \in \mathcal{R}$ ), $\delta$ of the form $y$, and $\forall x \in X,\{\bar{x}\} \mathcal{R}^{\prime} \overrightarrow{X y},\{x\} \mathcal{R}^{\prime} \bar{x}$, and so by the admissibility of $E^{\prime}, \forall x \in X, x \in E^{\prime}$. Since $E=\left(E^{\prime} \cap \mathcal{A}\right), X \subseteq E$, and since $(X, y) \in \mathcal{R}$ then $\alpha$ is acceptable w.r.t. $E$. Note that we have also shown that $\overrightarrow{X y} \in E^{\prime}$ implies $X \subseteq E,(X, y) \in \mathcal{R}$.

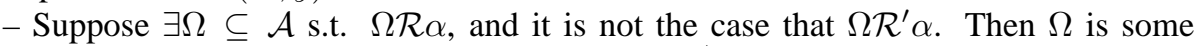
$Y, \alpha$ some $z$, expand $((Y, z)) \subseteq \mathcal{R}^{\prime}$. Hence $\{\overrightarrow{Y z}\} \mathcal{R}^{\prime} z$, and by admissibility of $E^{\prime}$, $\exists \bar{y} \in E^{\prime}$ s.t. $\{\bar{y}\} \mathcal{R}^{\prime} \overrightarrow{Y z}$, where $y \in Y$. Since $\{y\} \mathcal{R}^{\prime} \bar{y}$, then by the admissibility of $E^{\prime}$ either: 1) $\exists X \subseteq E^{\prime}$ s.t. $X \mathcal{R}^{\prime} y$, and $(X, y) \in \mathcal{R}, X \subseteq E$, and so $\alpha(=z)$ is acceptable w.r.t. $E$ or; 2) $\exists \overrightarrow{X y} \in E^{\prime}$ s.t. $\{\overrightarrow{X y}\} \mathcal{R}^{\prime} y$, where $\operatorname{expand}((X, y)) \subseteq \mathcal{R}^{\prime}$ and $\forall x \in X$, $\{\bar{x}\} \mathcal{R}^{\prime} \overrightarrow{X y},\{x\} \mathcal{R}^{\prime} \bar{x}$, and so by the admissibility of $E^{\prime}, X \subseteq E^{\prime}$, and so $X \subseteq E, \alpha$ (= $z$ ) is acceptable w.r.t. $E$. Note we have also shown that $\bar{y} \in E^{\prime}$ implies $\exists X, X \subseteq E$, $(X, y) \in \mathcal{R}$.

Lemma 3 Let $\Delta^{\prime}=\left(\mathcal{A}^{\prime}, \mathcal{R}^{\prime}\right)$ be an expansion of the DungC framework $\Delta=(\mathcal{A}, \mathcal{R})$. Then for $s \in\{$ admissible, complete, preferred, grounded, stable $\}, E$ is an $s$ extension of $(\mathcal{A}, \mathcal{R})$ iff $E^{\prime}$ is an $s$ extension of $\left(\mathcal{A}^{\prime}, \mathcal{R}^{\prime}\right)$, where:

1. $\forall \alpha \in \mathcal{A}, \alpha \in E$ iff $\alpha \in E^{\prime}$

2. $\exists X \subseteq E,(X, y) \in \mathcal{R}$ iff $\overrightarrow{X y} \in E^{\prime}$, where $\operatorname{expand}((X, y)) \subseteq \mathcal{R}^{\prime}$

3. $\exists X \subseteq E,(X, y) \in \mathcal{R}$ iff $\bar{y} \in E^{\prime}$, where $\bar{y} \in \mathcal{A}^{\prime}$

\section{Proof:}

1. $s=$ admissible. 1.1 Left to right half follows from Lemma 1. 1.2. Right to left half follows from Lemma 2.

Let us define functions $f$ and $g$ s.t. 
For any admissible extension $E$ of $\Delta, E^{\prime}=h(E)$ as defined above.

For any admissible extension $E^{\prime}$ of $\Delta^{\prime}, E=g\left(E^{\prime}\right)$ as defined above.

We show that:

a) $h$ is monotonically strictly increasing in the sense that $\forall E, F$ s.t. $E$ and $F$ are admissible extensions of $\Delta$ and $E \subset F$, then $h(E) \subset h(F)$

Suppose $E$ and by $\mathbf{1 . 1}$ the corresponding admissible $E^{\prime}=h(E)$. Suppose $E \subset F$ and by 1.1 the corresponding admissible $F^{\prime}=h(F)$. It is obvious to see that $E^{\prime} \subset F^{\prime}$

b) $g$ is monotonically strictly increasing in the sense that $\forall E^{\prime}, F^{\prime}$ s.t. $E^{\prime}$ and $F^{\prime}$ are admissible extensions of $\Delta^{\prime}$ and $E^{\prime} \subset F^{\prime}$, then $g\left(E^{\prime}\right) \subset g\left(F^{\prime}\right)$

Suppose $E^{\prime}$ and by $\mathbf{1 . 2}$ the corresponding admissible $E=g\left(E^{\prime}\right)$. Suppose $E^{\prime} \subset F^{\prime}$. Then:

$\forall \alpha \in\left(F^{\prime}-E^{\prime}\right)$, if $\alpha \in \mathcal{A}$ or $\alpha$ is of the form $\bar{y}$ or $\overrightarrow{X y}$, then $\alpha \notin E$, respectively $\neg \exists X \subseteq E$ s.t. $(X, y) \in \mathcal{R}$, since otherwise, by application of 1.1 to $E$, we would have $\alpha \in E^{\prime}$, respectively $\bar{y}$ or $\overrightarrow{X y} \in E^{\prime}$.

By 1.2, let $F$ be the corresponding admissible extension of $\Delta$. Given i), $E \subset F$.

$2 s=$ complete.

2.1 Left to right half: Suppose $E$ is complete. Applying 1.1, $E^{\prime}$ is an admissible extension of $\Delta^{\prime}$, where $E=g\left(E^{\prime}\right)$. Suppose $E^{\prime}$ is not complete. Then $\exists \alpha \notin E^{\prime}, \alpha$ acceptable w.r.t. $E^{\prime}$, and so by Dung's fundamental lemma [27], $F^{\prime}=E^{\prime} \cup\{\alpha\}$ is admissible where $F^{\prime} \supset E^{\prime}$. Applying 1.2, $F=g\left(F^{\prime}\right)$ is an admissible extension of $\Delta$, where by b), $E \subset F$, contradicting $E$ is complete.

2.2 Right to left half: Suppose $E^{\prime}$ is complete. Applying 1.2, $E$ is an admissible extension of $\Delta$, where $E^{\prime}=h(E)$. Suppose $E$ is not complete. Then $\exists \alpha \notin E, \alpha$ acceptable w.r.t. $E$, and so by Dung's fundamental lemma, $F=E \cup\{\alpha\}$ is admissible where $F \supset E$. Applying 1.1, $F^{\prime}=h(F)$ is an admissible extension of $\Delta^{\prime}$, where by a), $E^{\prime} \subset F^{\prime}$, contradicting $E^{\prime}$ is complete.

\section{$3 s=$ preferred.}

3.1 Left to right half: Suppose $E$ is preferred. The proof now proceeds in the same way as 2.1, except that supposing $E^{\prime}$ is not preferred immediately implies $\exists F^{\prime} \supset E^{\prime}$ s.t. $F^{\prime}$ is admissible.

3.2 Right to left half: Suppose $E^{\prime}$ is preferred. The proof now proceeds in the same way as 2.2, except that supposing $E$ is not preferred immediately implies $\exists F \supset E$ s.t. $F$ is admissible.

\section{$3 s=$ grounded.}

4.1 Left to right half: Suppose $E$ is grounded. Applying 2.1, $E^{\prime}$ is a complete extension of $\Delta^{\prime}$, where $E=g\left(E^{\prime}\right)$. Suppose $E^{\prime}$ is not grounded. Then $\exists F^{\prime} \subset E^{\prime}, F^{\prime}$ is complete. Applying 2.2, $F=g\left(F^{\prime}\right)$ is a complete and so admissible extension of $\Delta$, where by b), $F \subset E$, contradicting $E$ is grounded.

4.2 Right to left half: Suppose $E^{\prime}$ is grounded. Applying 2.2, $E$ is a complete extension of $\Delta$, where $E^{\prime}=h(E)$. Suppose $E$ is not grounded. Then $\exists F \subset E, F$ is complete. Applying 2.1, $F^{\prime}=h(F)$ is a complete and so admissible extension of $\Delta^{\prime}$, where by a), $F^{\prime} \subset E^{\prime}$, contradicting $E^{\prime}$ is grounded.

\section{$5 s$ = stable:}

5.1 Left to right half: Suppose $E$ is stable. Applying 2.1, $E^{\prime}$ is complete. Suppose $\alpha \in \mathcal{A}, \alpha \notin E^{\prime}$. Then $\alpha \notin E, \exists \Gamma \subseteq E$ s.t. $(\Gamma, \alpha) \in \mathcal{R}$. Since $E \subseteq E^{\prime}, \Gamma \subseteq E^{\prime}$. Suppose $(\Gamma, \alpha) \notin \mathcal{R}^{\prime}$. Then $(\Gamma, \alpha)=(X, y)$, expand $((X, y)) \subseteq \mathcal{R}^{\prime}$ and so $\overrightarrow{X y} \in E^{\prime}$, 
$(\overrightarrow{X y}, y) \in \mathcal{R}^{\prime}$. Suppose $\exists \alpha \in\left(\mathcal{A}^{\prime}-\mathcal{A}\right), \alpha \notin E^{\prime}, \neg \exists \Gamma \subseteq E^{\prime}$ s.t. $(\Gamma, \alpha) \in \mathcal{R}^{\prime}$. Then either:

$\alpha$ is of the form $\bar{y}$, in which case $\{y\} \mathcal{R}^{\prime} \bar{y}, y \notin E^{\prime}$. But then we have already shown that $y \in \mathcal{A}$ is attacked by some subset of $E^{\prime}$, and so $\bar{y}$ is acceptable w.r.t. $E^{\prime}$, contradicting $E^{\prime}$ is complete;

$\alpha$ is of the form $\overrightarrow{X y}$, in which case $\forall x \in X,\{\bar{x}\} \mathcal{R}^{\prime} \overrightarrow{X y}, \bar{x} \notin E^{\prime}$. For any such $\bar{x}, x \in E^{\prime}$, and since $\{x\} \mathcal{R}^{\prime} \bar{x}$, then $\overrightarrow{X y}$ is acceptable w.r.t. $E^{\prime}$, contradicting $E^{\prime}$ is complete.

5.2 Right to left half: Suppose $E^{\prime}$ is a stable extension. Applying 2.2, $E$ is complete. Suppose some $\alpha \in \mathcal{A}, \alpha \notin E$. Then $\alpha \notin E^{\prime}, \exists \Gamma \subseteq E^{\prime}$ s.t. $(\Gamma, \alpha) \in \mathcal{R}^{\prime}$. Suppose it is not the case that $\Gamma \subseteq E$ and $(\Gamma, \alpha) \in \mathcal{R}$. Then $\Gamma$ is some $\{\overrightarrow{X y}\}, \alpha$ some $y$, and by 2.2, $\exists X \subseteq E,(X, y) \in \mathcal{R}$.

Notice that since the definitions of conflict free, acceptability and the extensions of a standard Dung framework are a special case of DungC frameworks (i.e., the case where every attack originates from a singleton set of arguments), then corollaries of the above results establish the same correspondences for Dung frameworks and their expansions.

Corollary 1 Let $\Delta^{\prime}=\left(\mathcal{A}^{\prime}, \mathcal{R}^{\prime}\right)$ be an expansion of the Dung framework $\Delta=(\mathcal{A}, \mathcal{R})$. Then for $s \in\{$ admissible, complete, preferred, grounded, stable $\}, E$ is an $s$ extension of $(\mathcal{A}, \mathcal{R})$ iff $E^{\prime}$ is an $s$ extension of $\left(\mathcal{A}^{\prime}, \mathcal{R}^{\prime}\right)$, where:

1. $\forall \alpha \in \mathcal{A}, \alpha \in E$ iff $\alpha \in E^{\prime}$

2. $\exists x \in E,(x, y) \in \mathcal{R}$ iff $\overrightarrow{x y} \in E^{\prime}$, where expand $((x, y)) \in \mathcal{R}^{\prime}$

3. $\exists x \in E,(x, y) \in \mathcal{R}$ iff $\bar{y} \in E^{\prime}$, where $\bar{y} \in \mathcal{A}^{\prime}$

\subsection{Proofs for Section 2.1.2}

Proposition 1 Let $\Delta=(\mathcal{A}, \mathcal{R})$, and for $s \in\{$ admissible, complete, grounded, preferred, stable $\}$, let $E$ be an $s$ extension of $\Delta$. Then there exists an $s$ labelling $\mathcal{L}$ of $\Delta$ such that $\operatorname{in}(\mathcal{L})=E$, and out $(\mathcal{L})=(E+) \cup(E-)$.

Proof: Obvious, given Theorem 1 and Definition 6.

\subsection{Proofs for Sections 3.2 and 3.3}

Since DungC frameworks with collective attacks are a straightforward generalisation of Dung frameworks, we will establish a series of results for DungC frameworks, and then state Section 3.2's results as corollaries of the results shown for DungC frameworks. In what follows we will make use of the following generalisation of Notation 1.

Notation 3 Let $(\mathcal{A}, \mathcal{R})$ be a DungC framework, and $E \subseteq \mathcal{A}$.

- $\overrightarrow{E+}$ denotes the set of attacks originating from sets of arguments in $E$ : $\overrightarrow{E+}=\{(B, y) \mid B \subseteq E, B \mathcal{R} y\}$

- $E+$ denotes the set of arguments attacked by sets of arguments $B \subseteq E$ : $E+=\{y \mid B \subseteq E, B \mathcal{R} y\}$

- $E$ - denotes the set of sets of arguments that attack arguments in $E$ : $E-=\{B \mid B \mathcal{R} x, x \in E\}$ 
Lemma 4 Let $\Delta_{M}=\left(\mathcal{A}_{\mathcal{M}}, \mathcal{R}_{\mathcal{M}}, \mathcal{C}, \mathcal{L}_{\mathcal{M}}, \mathcal{D}_{d c}\right)$ be the $M A F$ of a DungC framework $\Delta=(\mathcal{A}, \mathcal{R})$. Then for $s \in\{$ admissible, complete, grounded, preferred, stable $\}, E$ is an $s$ extension of $\Delta$ iff $E^{\prime}$ is an $s$ extension of $\Delta_{M}$, where:

1. $X \mathcal{R} y \in \overrightarrow{E+}$ iff $(X \operatorname{def} y) \in E^{\prime}$

2. $y \in E+$ iff $(r-y) \in E^{\prime}$

3. $x \in E$ iff $(j-x) \in E^{\prime}$

Proof: Let $\Delta^{*}=\left(\mathcal{A}^{*}, \mathcal{R}^{*}\right)$ be the expansion of $\Delta=(\mathcal{A}, \mathcal{R})$ such that $\forall(X, y) \in \mathcal{R}$, $\operatorname{expand}((X, y)) \subseteq \mathcal{R}^{*}$. Let $\Delta^{* *}=\left(\mathcal{A}^{* *}, \mathcal{R}^{* *}\right)$ be $\Delta^{*}$ 's augmentation, defined as follows:

$$
\mathcal{A}^{* *}=\mathcal{A}^{*} \cup\{\bar{y} \mid y \in \mathcal{A}\} \text { and } \mathcal{R}^{* *}=\mathcal{R}^{*} \cup\{(\{y\}, \bar{y}) \mid y \in \mathcal{A}\}
$$

In other words $\Delta^{* *}$ is defined by additionally including attacks $(\{y\}, \bar{y})$ for those $y$ that are not a member of some set $Y$ s.t. $(Y, x) \in \mathcal{R}$, and are thus are not obtained by expanding the attacks in $\Delta$. The following holds:

For $s \in\{$ admissible, complete, grounded, preferred, stable $\}, E^{*}$ is an $s$ extension of $\Delta^{*}$ iff $E^{* *}$ is an $s$ extension of $\Delta^{* *}$, where $E^{*} \subseteq E^{* *}$, and $\left(E^{* *}-E^{*}\right)=\left\{\bar{y} \mid \overrightarrow{X y} \in E^{*}, \bar{y} \notin \mathcal{A}^{*}\right\}$

(i) follows given that no $\mathcal{R}^{* *}$ attacks originate from the extra $\bar{y}$ arguments in $\left(\mathcal{A}^{* *}-\right.$ $\mathcal{A}^{*}$ ), and so $\forall \alpha \in E^{*} \cap E^{* *}, \alpha$ is acceptable w.r.t. $E^{*}$ iff $\alpha$ is acceptable w.r.t. $E^{* *}$, and since $\overrightarrow{X y} \in E^{*}$ iff $\overrightarrow{X y} \in E^{* *},\{\overrightarrow{X y}\} \mathcal{R}^{*} y$ iff $\{\overrightarrow{X y}\} \mathcal{R}^{* *} y$, and $\forall y,\{y\} \mathcal{R}^{* *} \bar{y}$, then each $\bar{y} \in\left(E^{* *}-E^{*}\right)$ is acceptable w.r.t. $E^{* *}$.

It should now be obvious to see that the argument graphs $\Delta_{M}$ and $\Delta^{* *}$ are isomorphic, where $f$ is a bijective function from $\mathcal{A}_{\mathcal{M}}$ to $\mathcal{A}^{* *}$ s.t.

- $f((j-x))=x\left(\right.$ where $\left.x \in \mathcal{A}, \mathcal{A}^{*}, \mathcal{A}^{* *}\right)$

- $f((r-y))=\bar{y}$

- $f((X \operatorname{def} y))=\overrightarrow{X y}$

and $g$ is a bijective function from $\mathcal{R}_{\mathcal{M}}$ to $\mathcal{R}^{* *}$ s.t. $g(\alpha, \beta)=(f(\alpha), f(\beta))$.

To see that this is so, observe that $\left(\mathcal{A}_{\mathcal{M}}, \mathcal{R}_{\mathcal{M}}\right)$ is effectively obtained by replacing every $x \in \mathcal{A}$ by $(j-x)$, and then every attack $(X, y)$ is expanded, interspersing $(j-x) \mathcal{R}_{\mathcal{M}}(r-x),(r-x) \mathcal{R}_{\mathcal{M}}(X \operatorname{def} y)$ for all $x \in X$, and $(X \operatorname{def} y) \mathcal{R}_{\mathcal{M}}(j-y)$, and for every $(j-x)$, the attack $(j-x) \mathcal{R}_{\mathcal{M}}(r-x)$ is added.

We now prove the main result:

- By lemma 3, $E$ is an $s$ extension of $\Delta$ iff $E^{*}$ is an $s$ extension of the expansion $\Delta^{*}$, where $\forall \alpha \in \mathcal{A}, \alpha \in E$ iff $\alpha \in E^{*}, X \subseteq E$ and $(X, y) \in \mathcal{R}$ iff $\overrightarrow{X y} \in E^{*}$ (recall that every attack $(X, y)$ is expanded in $\left.\Delta^{*}\right)$ and $\bar{y} \in E^{*}$ s.t. $\bar{y} \in \mathcal{A}^{*}$.

- Given (i), $E$ is an $s$ extension of $\Delta$ iff $E^{* *}$ is an $s$ extension of the augmentation $\Delta^{* *}$ of $\Delta^{*}$, where $\forall \alpha \in \mathcal{A}, \alpha \in E$ iff $\alpha \in E^{* *}, X \subseteq E$ and $(X, y) \in \mathcal{R}$ iff $\overrightarrow{X y}, \bar{y} \in E^{* *}$, (given that $\overrightarrow{X y} \in E^{* *}$ implies $\bar{y} \in E^{* *}$ ).

- By the isomorphism of $\Delta_{M}$ and $\Delta^{* *}: E$ is an $s$ extension of $\Delta$ iff $E^{\prime}$ is an $s$ extension of $\Delta_{M}$, where $\forall x \in \mathcal{A}, x \in E$ iff $(j-x) \in E^{\prime}, X \subseteq E,(X, y) \in \mathcal{R}$ (i.e., $X \mathcal{R} y \in \overrightarrow{E+}$ and $y \in E+)$ iff $((X \operatorname{def} y)),(r-y) \in E^{\prime}$. 
Corollary 2 Let $\Delta_{M}=\left(\mathcal{A}_{\mathcal{M}}, \mathcal{R}_{\mathcal{M}}, \mathcal{C}, \mathcal{L}_{\mathcal{M}}, \mathcal{D}_{d}\right)$ be the $M A F$ of a Dung framework $\Delta=(\mathcal{A}, \mathcal{R})$. Then for $s \in\{$ admissible, complete, grounded, preferred, stable $\}, E$ is an $s$ extension of $\Delta$ iff $E^{\prime}$ is an $s$ extension of $\Delta_{M}$, where:

1. $x \mathcal{R} y \in \overrightarrow{E+}$ iff $(x \operatorname{def} y) \in E^{\prime}$

2. $y \in E+$ iff $(r-y) \in E^{\prime}$

3. $x \in E$ iff $(j-x) \in E^{\prime}$

Theorem 2 Let $\Delta_{M}=\left(\mathcal{A}_{\mathcal{M}}, \mathcal{R}_{\mathcal{M}}, \mathcal{C}, \mathcal{L}_{\mathcal{M}}, \mathcal{D}_{d}\right)$ be the $M A F$ of a Dung framework $(\mathcal{A}, \mathcal{R})$. Then for $s \in\{$ complete, grounded, preferred, stable $\},(j-x) \in \mathcal{A}_{\mathcal{M}}$ is a credulously, respectively sceptically, justified argument of $\Delta_{M}$ under the $s$ semantics, iff $x \in \mathcal{A}$ is a credulously, respectively sceptically, justified argument of $\Delta$ under the $s$ semantics.

Proof Follows from Corollary 2.

Proposition 2 Let $\Delta_{\mathcal{M}}=\left(\mathcal{A}_{\mathcal{M}}, \mathcal{R}_{\mathcal{M}}, \mathcal{C}, \mathcal{L}_{\mathcal{M}}, \mathcal{D}_{d}\right)$ be the $M A F$ of a Dung framework $\Delta=(\mathcal{A}, \mathcal{R})$. For $s \in\{$ admissible, complete, grounded, preferred, stable $\}$ :

There exists an $s$ labelling $\mathcal{L}$ of $\Delta$ iff there exists an $s$ extension $E$ of $\Delta_{\mathcal{M}}$ such that:

$$
\begin{aligned}
& \text { 1. } x \in \operatorname{in}(\mathcal{L}) \text { iff }(j-x) \in E \\
& \text { 2. } y \in \operatorname{out}(\mathcal{L}) \text { iff }(r-y) \in E
\end{aligned}
$$

\section{Proof:}

Left to right: Let $\mathcal{L}$ be an $s$ labelling of $\Delta$. By Theorem $1, E^{\prime}=\operatorname{in}(\mathcal{L})$ is an $s$ extension of $\Delta$. By Corollary 2, there is an $s$ extension $E$ of $\Delta_{\mathcal{M}}$, where $E=\left\{(j-x) \mid x \in E^{\prime}\right\}$ $\cup\left\{(x\right.$ def $\left.y) \mid x \mathcal{R} y \in \overrightarrow{E^{\prime}+}\right\} \cup\left\{(r-y) \mid y \in E^{\prime}+\right\}$. Hence, $x \in \operatorname{in}(\mathcal{L})$ implies $(j-x) \in E$, and since by Definition $6, y \in \operatorname{out}(\mathcal{L})$ implies $y \in E^{\prime}+$, then $y \in$ out $(\mathcal{L})$ implies $(r-y) \in E$.

Right to left: Let $E$ be an $s$ extension of $\Delta_{\mathcal{M}}$. Let $E^{\prime}=\{x \mid(j-x) \in E\}$ be the $s$ extension of $\Delta$ as defined in Corollary 2, where if $(r-y) \in E$ then $y \in E^{\prime}+$. By Proposition 1 there is an $s$ labelling $\mathcal{L}$ where $\operatorname{in}(\mathcal{L})=E^{\prime}$, out $(\mathcal{L})=E^{\prime}+$ (recall that $\left.E^{\prime}-\subseteq E^{\prime}+\right)$.

Theorem 3 Let $\Delta_{M}=\left(\mathcal{A}_{\mathcal{M}}, \mathcal{R}_{\mathcal{M}}, \mathcal{C}, \mathcal{L}_{\mathcal{M}}, \mathcal{D}_{d c}\right)$ be the $M A F$ of a DungC framework $\Delta=(\mathcal{A}, \mathcal{R})$. Then for $s \in\{$ complete, grounded, preferred, stable $\},(j-x) \in \mathcal{A}_{\mathcal{M}}$ is a credulously, respectively sceptically, justified argument of $\Delta_{M}$ under the $s$ semantics, iff $x \in \mathcal{A}$ is a credulously, respectively sceptically, justified argument of $\Delta$ under the $s$ semantics.

Proof: Follows from Lemma 4.

\subsection{Proofs for Sections 3.4 and 3.5}

In what follows we make use of the following notation:

Notation 4 Let $(\mathcal{A}$, defeat) be defined on the basis of $(\mathcal{A}, \mathcal{R}, \mathcal{P})$ as in Definition 9 , and let $E \subseteq \mathcal{A}$.

- $\overrightarrow{E+}$ denotes the set of defeats from arguments in $E$ :

$\overrightarrow{E+}=\{(x, y) \mid(x, y) \in$ defeat,$x \in E\}$ 
- $E+$ denotes the set of arguments defeated by arguments in $E$ :

$E+=\{y \mid(x, y) \in$ defeat, $x \in E\}$

- $E$ - denotes the set of arguments that defeat arguments in $E$ :

$E-=\{y \mid(y, x) \in$ defeat, $x \in E\}$

Lemma 5 Let $\left(\mathcal{A}_{\mathcal{P}}, \mathcal{R}_{\mathcal{P}}, \mathcal{C}, \mathcal{L}_{\mathcal{M}}, \mathcal{D}_{p}\right)$ be the $P-M A F$ of a $P A F(\mathcal{A}, \mathcal{R}, \mathcal{P})$. For $s \in\{$ admissible, complete, preferred, stable, grounded $\}$ :

$E$ is an $s$ extension of $(\mathcal{A}, \mathcal{R}, \mathcal{P})$ iff $E^{\prime} \cup\left\{(x P y) \mid x \gg_{\mathcal{P}} y\right\}$ is an $s$ extension of $\left(\mathcal{A}_{\mathcal{P}}, \mathcal{R}_{\mathcal{P}}\right)$, where:

1. $(x, y) \in \overrightarrow{E+}$ iff $(x \operatorname{def} y) \in E^{\prime}$

2. $y \in E+\operatorname{iff}(r-y) \in E^{\prime}$

3. $x \in E$ iff $(j-x) \in E^{\prime}$

Proof By Definition $9, E$ is an $s$ extension of $(\mathcal{A}, \mathcal{R}, \mathcal{P})$ iff $E$ is an $s$ extension of the Dung framework $(\mathcal{A}$, defeat $)$, where $(x, y) \in$ defeat iff $(x, y) \in \mathcal{R}$ and $\neg\left(y \gg_{\mathcal{P}} x\right)$. Let $\Delta_{M}=\left(\mathcal{A}_{\mathcal{M}}, \mathcal{R}_{\mathcal{M}}, \mathcal{C}, \mathcal{L}_{\mathcal{M}}, \mathcal{D}_{d}\right)$ be the $M A F$ of $(\mathcal{A}$, defeat $)$. By Corollary $2, E$ is an $s$ extension of $(\mathcal{A}$, defeat $)$ iff $E^{M}$ is an $s$ extension of $\left(\mathcal{A}_{\mathcal{M}}, \mathcal{R}_{\mathcal{M}}\right)$, where:

1. $(x, y) \in \overrightarrow{E+} \operatorname{iff}(x \operatorname{def} y) \in E^{M}$

2. $y \in E+\operatorname{iff}(r-y) \in E^{M}$

3. $x \in E$ iff $(j-x) \in E^{M}$

Hence it suffices to show the following result:

$$
E^{M} \text { is an } s \text { extension of }\left(\mathcal{A}_{\mathcal{M}}, \mathcal{R}_{\mathcal{M}}\right) \text { iff } E^{P}=E^{M} \cup\left\{(x P y) \mid x \gg_{\mathcal{P}} y\right\} \text { is }
$$
an $s$ extension of $\left(\mathcal{A}_{\mathcal{P}}, \mathcal{R}_{\mathcal{P}}\right)$

Firstly, note that it is straightforward to show that:

i) $\mathcal{A}_{\mathcal{M}} \subseteq \mathcal{A}_{\mathcal{P}}$, where $\mathcal{A}_{\mathcal{P}}-\mathcal{A}_{\mathcal{M}}=\left\{(x P y) \mid x \gg_{\mathcal{P}} y\right\} \cup\left\{(y \operatorname{def} x) \mid x \gg_{\mathcal{P}} y, y \mathcal{R} x\right\}$

ii) $\mathcal{R}_{\mathcal{M}} \subseteq \mathcal{R}_{\mathcal{P}}$, where $\mathcal{R}_{\mathcal{P}}-\mathcal{R}_{\mathcal{M}}=$

$\left\{((x P y),(y \operatorname{def} x)),((r-y),(y \operatorname{def} x)),((y \operatorname{def} x),(j-x)) \mid x \gg_{\mathcal{P}} y, y \mathcal{R} x\right\}$

1.1 Left to right half for $s=$ admissible: Assume $E^{M}$ is an admissible extension of $\left(\mathcal{A}_{\mathcal{M}}, \mathcal{R}_{\mathcal{M}}\right)$ and $E^{P}$ defined as above. We show that every $\alpha \in E^{P}$ is acceptable w.r.t. $E^{P}$ :

1.1.1 Since each $(x P y) \in \mathcal{A}_{\mathcal{P}}$ is not attacked by any argument, then each $(x P y) \in$ $\mathcal{A}_{\mathcal{P}}$ is acceptable w.r.t. $E^{P}$.

1.1.2 Suppose $\alpha \in E^{M}$ and so $\alpha \in E^{P}$.

- Suppose $(\beta, \alpha) \in \mathcal{R}_{\mathcal{P}}$ and $(\beta, \alpha) \in \mathcal{R}_{\mathcal{M}}$. Hence, $\exists \gamma \in E^{M},(\gamma, \beta) \in \mathcal{R}_{\mathcal{M}}$, and since $\mathcal{R}_{\mathcal{M}} \subseteq \mathcal{R}_{\mathcal{P}},(\gamma, \beta) \in \mathcal{R}_{\mathcal{P}}$, where $\gamma \in E^{P}$ by definition of $E^{P}$.

- Suppose $(\beta, \alpha) \in \mathcal{R}_{\mathcal{P}}$ and $(\beta, \alpha) \notin \mathcal{R}_{\mathcal{M}}$. Since $\alpha \in E^{M}$, then by i) and ii) it must be the case that $\alpha$ is of the form $(j-x), \beta$ is of the form $(y \operatorname{def} x)$, and $(x P y) \mathcal{R}_{\mathcal{P}}(y \operatorname{def} x)$, where $(x P y) \in E^{P}$.

1.2 Right to left half for $s=$ admissible: Assume $E^{P}$ is an admissible extension of $\left(\mathcal{A}_{\mathcal{P}}, \mathcal{R}_{\mathcal{P}}\right)$ and $E^{M}$ defined as above. We show that every $\alpha \in E^{M}$ is acceptable w.r.t. $E^{M}$. Suppose some $(\beta, \alpha) \in \mathcal{R}_{\mathcal{M}}$. Hence $(\beta, \alpha) \in \mathcal{R}_{\mathcal{P}}$ and $\exists \gamma \in E^{P}, \gamma \mathcal{R}_{\mathcal{P}} \beta$. 
1.2.1 Suppose $\gamma \in E^{M},(\gamma, \beta) \notin \mathcal{R}_{\mathcal{M}}$. By i) and ii), $\gamma$ must be of the form $(r-y), \beta$ of the form $(y \operatorname{def} x)$, and $(y \operatorname{def} x) \notin \mathcal{A}_{\mathcal{M}}$, contradicting $(\beta, \alpha) \in \mathcal{R}_{\mathcal{M}}$.

1.2.2 Suppose $\gamma \notin E^{M}$, in which case $\gamma$ is of the form $(x P y), \beta$ is of the form ( $y$ def $x)$, and by i), $(y \operatorname{def} x) \notin \mathcal{A}_{\mathcal{M}}$, contradicting $(\beta, \alpha) \in \mathcal{R}_{\mathcal{M}}$.

- $s \in\{$ complete, grounded, preferred $\}$. We define functions $f$ and $g$ s.t.

For any admissible extension $E^{M}$ of $\Delta^{M}=\left(\mathcal{A}_{\mathcal{M}}, \mathcal{R}_{\mathcal{M}}\right), E^{P}=h\left(E^{M}\right)$ as defined above.

For any admissible extension $E^{P}$ of $\Delta^{P}=\left(\mathcal{A}_{\mathcal{P}}, \mathcal{R}_{\mathcal{P}}\right), E^{M}=g\left(E^{P}\right)$ as defined above.

We show that:

a) $h$ is monotonically strictly increasing in the sense that $\forall E^{M}, F^{M}$ s.t. $E^{M}$ and $F^{M}$ are admissible extensions of $\Delta^{M}$ and $E^{M} \subset F^{M}$, then $h\left(E^{M}\right) \subset h\left(F^{M}\right)$.

Suppose $E^{M}$ and by $\mathbf{1 . 1}$ the corresponding admissible $E^{P}=h\left(E^{M}\right)$. Suppose $E^{M} \subset$ $F^{M}$ and by 1.1 the corresponding admissible $F^{P}=h\left(F^{M}\right)$. It is obvious to see that $E^{P} \subset F^{P}$

b) $g$ is monotonically strictly increasing in the sense that $\forall E^{P}, F^{P}$ s.t. $E^{P}$ and $F^{P}$ are admissible extensions of $\Delta^{P}$ and $E^{P} \subset F^{P}$, then $g\left(E^{P}\right) \subset g\left(F^{P}\right)$.

Suppose $E^{P}$ and by $\mathbf{1 . 2}$ the corresponding admissible $E^{M}=g\left(E^{P}\right)$. Suppose $E^{P} \subset$ $F^{P}$, where by 1.1.1, $\forall \alpha \in\left(F^{P}-E^{P}\right), \alpha$ is not of the form $(x P y)$. Hence, by $\mathbf{1 . 2}$, $F^{M}$ is the corresponding admissible extension of $\Delta$, where $E^{M} \subset F^{M}$.

Given a) and b), the result is shown to hold for $s \in\{$ complete, grounded, preferred $\}$ in exactly the same way as in Lemma 3.

- Left to right half for $s=$ stable: Assume $E^{M}$ is stable, and $E^{P}$ defined as above. Suppose $\exists \alpha \notin E^{P}$ s.t. no argument in $E^{P} \mathcal{R}_{\mathcal{P}}$ attacks $\alpha$. Then $\alpha \in \mathcal{A}_{\mathcal{M}}$, since otherwise $\alpha$ is of the form $(x P y)$, contradicting $(x P y) \in E^{P}$, or $\alpha$ is of the form $(y \operatorname{def} x)$, where $(x P y) \mathcal{R}_{\mathcal{P}}(y \operatorname{def} x)$, contradicting no argument in $E^{P} \mathcal{R}_{\mathcal{P}}$ attacks $\alpha$. Hence, $\alpha \notin E^{M}$ (since otherwise $\alpha \in E^{P}$ by definition of $E^{P}$ ), and since $\mathcal{R}_{\mathcal{M}} \subseteq \mathcal{R}_{\mathcal{P}}$, $\alpha$ is not $\mathcal{R}_{\mathcal{M}}$ attacked by any argument in $E^{M}$, contradicting $E^{M}$ is stable.

Right to left half for $s=$ stable: Assume $E^{P}$ is stable. If $E^{M}$ is not stable then $\exists \beta \in$ $\mathcal{A}_{\mathcal{M}}, \beta \notin E^{M}$ s.t. no argument in $E^{M} \mathcal{R}_{\mathcal{M}}$ attacks $\beta$. Since $\mathcal{A}_{\mathcal{M}} \subseteq \mathcal{A}_{\mathcal{P}}$, then $\beta \in \mathcal{A}_{\mathcal{P}}$. Since $E^{M} \subseteq E^{P}$, no argument in $E^{P}-E^{M}$ is in $\mathcal{A}_{\mathcal{M}}$, and $E^{P}$ is stable, then $\beta \notin E^{P}$ and there is an argument $\alpha$ in $E^{P}$ that $\mathcal{R}_{\mathcal{P}}$ attacks $\beta . \alpha \in E^{P}$ is either: - an argument of the form $(x P y)$, in which case $\beta$ is of the form $(y \operatorname{def} x)$. But then by i) and ii), $(y \operatorname{def} x) \notin \mathcal{A}_{\mathcal{M}}$, contradicting $\beta \in \mathcal{A}_{\mathcal{M}}$.

- not of the form $(x P y)$, in which case $\alpha \in E^{M}$. By assumption that no argument in $E^{M} \mathcal{R}_{\mathcal{M}}$ attacks $\beta$, and by i) and ii $), \alpha \mathcal{R}_{\mathcal{P}} \beta=(y \operatorname{def} x) \mathcal{R}_{\mathcal{P}}(j-x)$ or $(r-x) \mathcal{R}_{\mathcal{P}}(y \operatorname{def} x)$, where $(y \operatorname{def} x) \notin \mathcal{A}_{\mathcal{M}}$, contradicting $\alpha \in \mathcal{A}_{\mathcal{M}}$ and $\beta \in \mathcal{A}_{\mathcal{M}}$ respectively.

Theorem 4 Let $\Delta_{M}=\left(\mathcal{A}_{\mathcal{M}}, \mathcal{R}_{\mathcal{M}}, \mathcal{C}, \mathcal{L}_{\mathcal{M}}, \mathcal{D}_{p}\right)$ be the $P-M A F$ of a $P A F \Delta=$ $(\mathcal{A}, \mathcal{R}, \mathcal{P})$. Then for $s \in\{$ complete, grounded, preferred, stable $\},(j-x) \in \mathcal{A}_{\mathcal{M}}$ is a credulously, respectively sceptically, justified argument of $\Delta_{M}$ under the $s$ semantics, iff $x \in \mathcal{A}$ is a credulously, respectively sceptically, justified argument of $\Delta$ under the $s$ semantics.

Proof Since every complete (and so grounded, preferred and stable) extension of $\left(\mathcal{A}_{\mathcal{M}}\right.$, $\left.\mathcal{R}_{\mathcal{M}}\right)$ contains the set $\left\{(x P y) \mid x \gg_{\mathcal{P}} y\right\}$, then the theorem follows from Lemma 5. 
Lemma 6 Let $\Delta_{V}=\left(\mathcal{A}_{\mathcal{V}}, \mathcal{R}_{\mathcal{V}}, \mathcal{C}, \mathcal{L}_{\mathcal{M}}, \mathcal{D}_{v}\right)$ be the $V-M A F$ of an $a V A F \Delta=(\mathcal{A}, \mathcal{R}, V$, $v a l, a)$. Let the extensions of $\Delta$ be the extensions of $\left(\mathcal{A}\right.$, defeat $\left._{\mathrm{a}}\right)$, where $x$ defeats $_{\mathrm{a}} y$ iff $x \mathcal{R} y$, and $\neg\left(\operatorname{val}(y)>_{\mathrm{a}} \operatorname{val}(x)\right)$ (as defined in Definition 10). Let $\overrightarrow{E+}, E+$ be defined as in Notation 4 . Then, for $s \in\{$ admissible, complete, preferred, stable, grounded $\}$ :

$E$ is an $s$ extension of $\Delta$ iff $E^{\prime} \cup\left\{\left(x_{v} P y_{v^{\prime}}\right) \mid v>_{a} v^{\prime}\right\} \cup\left\{\left(>_{a}\right)\right\}$ is an $s$ extension of $\left(\mathcal{A}_{\mathcal{V}}, \mathcal{R}_{\mathcal{V}}\right)$, where:

1. $(x, y) \in \overrightarrow{E+}$ iff $(x \operatorname{def} y) \in E^{\prime}$

2. $y \in E+\operatorname{iff}(r-y) \in E^{\prime}$

3. $x \in E$ iff $(j-x) \in E^{\prime}$

Proof Let $\left(\mathcal{A}_{\mathcal{M}}, \mathcal{R}_{\mathcal{M}}, \mathcal{C}, \mathcal{L}_{\mathcal{M}}, \mathcal{D}_{d}\right)$ be the Dung $M A F$ of $\left(\mathcal{A}\right.$, defeat $\left.{ }_{\mathrm{a}}\right)$. By Corollary 2, $E$ is an $s$ extension of $\left(\mathcal{A}\right.$, defeat $\left.t_{\mathrm{a}}\right)$ iff $E^{M}$ is an $s$ extension of $\left(\mathcal{A}_{\mathcal{M}}, \mathcal{R}_{\mathcal{M}}\right)$, where:

1. $(x, y) \in \overrightarrow{E+} \operatorname{iff}(x \operatorname{def} y) \in E^{M}$

2. $y \in E+\operatorname{iff}(r-y) \in E^{M}$

3. $x \in E$ iff $(j-x) \in E^{M}$

Hence, letting $E^{M}=E^{\prime}$, it suffices to show that $E^{M}$ is an $s$ extension of $\left(\mathcal{A}_{\mathcal{M}}, \mathcal{R}_{\mathcal{M}}\right)$ iff $E^{V}=E^{M} \cup\left\{\left(x_{v} P y_{v^{\prime}}\right) \mid v>_{a} v^{\prime}\right\} \cup\left\{\left(>_{a}\right)\right\}$ is an $s$ extension of $\left(\mathcal{A}_{\mathcal{V}}, \mathcal{R}_{\mathcal{V}}\right)$.

Firstly, it is straightforward to show that:

i) $\mathcal{A}_{\mathcal{M}} \subseteq \mathcal{A}_{\mathcal{V}}$, where:

$\mathcal{A}_{\mathcal{V}}-\mathcal{A}_{\mathcal{M}}=\left\{>_{\mathrm{a}}\right\} \cup\left\{\left(x_{v} P y_{v^{\prime}}\right) \mid\left(x_{v} P y_{v^{\prime}}\right) \in \mathcal{A}_{\mathcal{V}}\right\} \cup\left\{(y \operatorname{def} x) \mid \operatorname{val}(x)>_{\mathrm{a}} \operatorname{val}(y)\right\}$

i.e., the set of arguments $\mathcal{A}_{\mathcal{V}}$ extends $\mathcal{A}_{\mathcal{M}}$ with the audience argument $>_{\mathrm{a}}$, all value preference arguments, and the arguments $(y \operatorname{def} x)$ that by definition of $\Delta_{V}$ are attacked by value preference arguments endorsed by $a$.

ii) $\mathcal{R}_{\mathcal{M}} \subseteq \mathcal{R}_{\mathcal{V}}$, where $\mathcal{R}_{\mathcal{V}}-\mathcal{R}_{\mathcal{M}}$ is the set of attacks $\left(\left(x_{v} P y_{v^{\prime}}\right),\left(y_{v^{\prime}} P x_{v}\right)\right)$ between value preference arguments, all attacks $\left(\left(>_{a}\right),\left(y_{v}, P x_{v}\right)\right)$ from the audience argument to value preference arguments, all attacks $\left(\left(x_{v} P y_{v^{\prime}}\right),(y\right.$ def $\left.x)\right)$ from value preference to attack arguments, and incoming and outgoing attacks to and from arguments $(y$ def $x$ ) that do not appear in $\mathcal{A}_{\mathcal{M}}$ given that $\operatorname{val}(x)>_{\mathrm{a}} \operatorname{val}(y)$ ), i.e.:

$$
\left\{((r-y),(y \operatorname{def} x)),((y \operatorname{def} x),(j-x)) \mid \operatorname{val}(x)>>_{\mathrm{a}} \operatorname{val}(y)\right\}
$$

iii) $E^{V}$ contains the audience argument $\left(>_{a}\right)$ and partitions the value preference arguments, so that for every $\left(y_{v^{\prime}} P x_{v}\right) \notin E^{V},\left(y_{v^{\prime}} P x_{v}\right)$ is attacked by $\left(>_{a}\right)$ (it is not the case that $\left.v^{\prime}>_{\mathrm{a}} v\right)$ thus reinstating every audience endorsed $\left(x_{v} P y_{v^{\prime}}\right) \in E^{V}$ against the attack by $\left(y_{v^{\prime}} P x_{v}\right)$.

Left to right half for $s=$ admissible: Assume $E^{M}$ is an admissible extension of $\left(\mathcal{A}_{\mathcal{M}}\right.$, $\mathcal{R}_{\mathcal{M}}$ ) and $E^{V}$ defined as above. We show that every $\alpha \in E^{V}$ is acceptable w.r.t. $E^{V}$ :

- Let $\alpha=\left(>_{a}\right)$. Then $\left(>_{a}\right)$ is acceptable w.r.t. $E^{V}$ since $>_{a}$ is not attacked by any argument, and by iii), all $\left(x_{v} P y_{v^{\prime}}\right) \in E^{V}$ are acceptable w.r.t. $E^{V}$.

- Suppose $\alpha \in E^{M} \cap E^{V}$.

- Suppose $(\beta, \alpha) \in \mathcal{R}_{\mathcal{V}}$ and $(\beta, \alpha) \in \mathcal{R}_{\mathcal{M}}$. Hence, $\exists \gamma \in E^{M},(\gamma, \beta) \in \mathcal{R}_{\mathcal{M}}$, and since $\mathcal{R}_{\mathcal{M}} \subseteq \mathcal{R}_{\mathcal{V}},(\gamma, \beta) \in \mathcal{R}_{\mathcal{V}}$, where $\gamma \in E^{V}$ by definition of $E^{V}$.

- Suppose $(\beta, \alpha) \in \mathcal{R}_{\mathcal{V}}$ and $(\beta, \alpha) \notin \mathcal{R}_{\mathcal{M}}$. Then by i) and ii) it must be the case that $\alpha$ is of the form $(j-x), \beta$ is of the form $(y \operatorname{def} x)$, where $\operatorname{val}(x)>_{\mathrm{a}} \operatorname{val}(y)$, and $(j-x)$ 
is acceptable w.r.t. $E^{V}$ given $\left(x_{v} P y_{v^{\prime}}\right) \mathcal{R}_{\mathcal{V}}(y$ def $x),\left(x_{v} P y_{v^{\prime}}\right) \in E^{V}$.

Right to left half for $s=$ admissible: Assume $E^{V}$ is an admissible extension of $\left(\mathcal{A}_{\mathcal{V}}\right.$, $\left.\mathcal{R}_{\mathcal{V}}\right)$ and $E^{M}$ defined as above. We show that every $\alpha \in E^{M}$ is acceptable w.r.t. $E^{M}$. Suppose $(\beta, \alpha) \in \mathcal{R}_{\mathcal{M}}$. Hence $\beta \mathcal{R}_{\mathcal{V}} \alpha$ and $\exists \gamma \in E^{V}, \gamma \mathcal{R}_{\mathcal{V}} \beta$.

- Suppose $\gamma \in E^{M},(\gamma, \beta) \notin \mathcal{R}_{\mathcal{M}}$. By i) and ii), $\gamma$ must be of the form $(r-y), \beta$ of the form $(y \operatorname{def} x)$, and $(y \operatorname{def} x) \notin \mathcal{A}_{\mathcal{M}}$, contradicting $(\beta, \alpha) \in \mathcal{R}_{\mathcal{M}}$.

- Suppose $\gamma \notin E^{M}$. Then $\gamma$ is of the form $\left(x_{v} P y_{v^{\prime}}\right)$ or $\left(>_{a}\right)$, and $\beta$ is of the form ( $y$ def $x$ ) or $\left(y_{v^{\prime}} P x_{v}\right)$. By i), in either case any such $\beta$ is not in $\mathcal{A}_{\mathcal{M}}$, contradicting $(\beta, \alpha) \in \mathcal{R}_{\mathcal{M}}$.

Left to right and right to left half for $s \in\{$ complete, grounded, preferred $\}$ : Let us define functions $f$ and $g$ s.t. for any admissible extension $E^{M}$ of $\Delta^{M}=\left(\mathcal{A}_{\mathcal{M}}, \mathcal{R}_{\mathcal{M}}\right)$, $E^{V}=h\left(E^{M}\right)$ as defined above, and for any admissible extension $E^{V}$ of $\Delta^{V}=\left(\mathcal{A}_{\mathcal{V}}\right.$, $\left.\mathcal{R}_{\mathcal{V}}\right), E^{M}=g\left(E^{V}\right)$ as defined above.

We show that a) $h$ is monotonically strictly increasing, and b) $g$ is monotonically strictly increasing, in the same way as in Lemma 5, substituting the superscript $V$ for $P$, and in the proof of $\mathbf{b})$ noting that $\forall \alpha \in\left(F^{V}-E^{V}\right)$, by definition of $E^{V}$ and $F^{V}, \alpha$ is not a value preference argument $\left(x_{v} P y_{v^{\prime}}\right)$ or the audience argument $\left(>_{a}\right)$. Given a) and b), the result is shown to hold for $s \in$ \{complete, grounded, preferred $\}$ in exactly the same way as in Lemma 3.

Left to right half for $s=$ stable: The proof proceeds in the same way as for the left to right half for $s=$ stable in Lemma 5 (substituting $\mathcal{R}_{\mathcal{V}}$ for $\mathcal{R}_{\mathcal{P}}$ ), except we show $\alpha \in \mathcal{A}_{\mathcal{M}}$ as follows. Suppose otherwise. Then:

$-\alpha$ is the audience argument $\left(>_{\mathrm{a}}\right)$, contradicting $\alpha \notin E^{V}$, or;

- by iii), $\alpha$ is a value preference argument $\left(y_{v^{\prime}} P x_{v}\right)$ attacked by $\left(>_{\mathrm{a}}\right)$, contradicting no argument in $E^{V} \mathcal{R}_{\mathcal{V}}$ attacks $\alpha$, or $\alpha$ is a value preference argument $\left(x_{v} P y_{v^{\prime}}\right)$ endorsed by $\left(>_{a}\right)$, contradicting $\alpha \notin E^{V}$, or;

$-\alpha$ is of the form $(y$ def $x)$ s.t. $\operatorname{val}(x)>_{a} \operatorname{val}(y)$, and so $(y$ def $x)$ is attacked by some $\left(x_{v} P y_{v^{\prime}}\right) \in E^{V}$ that is endorsed by $\left(>_{a}\right)$, contradicting no argument in $E^{V} \mathcal{R}_{\mathcal{V}}$ attacks $\alpha$.

Right to left half for $s=$ stable: Assume $E^{V}$ is stable and $E^{M}$ defined as above. By the right to left for $s=$ complete, $E^{M}$ is complete. If $E^{M}$ is not stable then $\exists \beta \in \mathcal{A}_{\mathcal{M}}$, $\beta \notin E^{M}$ s.t. no argument in $E^{M} \mathcal{R}_{\mathcal{M}}$ attacks $\beta$. Since $\mathcal{A}_{\mathcal{M}} \subseteq \mathcal{A}_{\mathcal{V}}$, then $\beta \in \mathcal{A}_{\mathcal{V}}$, and since $E^{M} \subseteq E^{V}$ and $E^{V}$ is stable, there is a $\alpha$ in $E^{V}$ that $\mathcal{R}_{\mathcal{V}}$ attacks $\beta$. Suppose $\alpha \in E^{V}-E^{M}$. Then $\alpha$ is of the form $\left(x_{v} P y_{v^{\prime}}\right)$ or $\left(>_{a}\right)$, and $\beta$ is of the form $(y$ def $x)$ or $\left(y_{v^{\prime}} P x_{v}\right)$, in either case contradicting $\beta \in \mathcal{A}_{\mathcal{M}}$. Suppose $\alpha \in E^{V}, \alpha \in E^{M}$, where by assumption that no argument in $E^{M} \mathcal{R}_{\mathcal{M}}$ attacks $\beta$, and by i) and ii), $\alpha \mathcal{R}_{\mathcal{V}} \beta$ $=(y \operatorname{def} x) \mathcal{R}_{\mathcal{V}}(j-x)$ or $(r-x) \mathcal{R}_{\mathcal{V}}(y \operatorname{def} x)$, and $(y \operatorname{def} x) \notin \mathcal{A}_{\mathcal{M}}$. Hence, the first case contradicts $\alpha \in \mathcal{A}_{\mathcal{M}}$, and the second case contradicts $\beta \in \mathcal{A}_{\mathcal{M}}$.

Theorem 5 Let $\Delta_{M}=\left(\mathcal{A}_{\mathcal{M}}, \mathcal{R}_{\mathcal{M}}, \mathcal{C}, \mathcal{L}_{\mathcal{M}}, \mathcal{D}_{v}\right)$ be the $V-M A F$ of an $a V A F \Delta=$ $(\mathcal{A}, \mathcal{R}, V, v a l, \mathrm{a})$. Then for $s \in$ \{complete, grounded, preferred, stable $\},(j-x) \in$ $\mathcal{A}_{\mathcal{M}}$ is a credulously, respectively sceptically, justified argument of $\Delta_{M}$ under the $s$ semantics, iff $x \in \mathcal{A}$ is a credulously, respectively sceptically, justified argument of $\Delta$ under the $s$ semantics

Proof Given that every complete (and so grounded, preferred and stable) extension of $\left(\mathcal{A}_{\mathcal{M}}, \mathcal{R}_{\mathcal{M}}\right)$ contains the set $\left\{\left(x_{v} P y_{v^{\prime}}\right) \mid v>_{\mathrm{a}} v^{\prime}\right\} \cup\left\{\left(>_{\mathrm{a}}\right)\right\}$, then the theorem follows immediately from Lemma 6. 
Lemma 7 Let $\Delta_{V}=\left(\mathcal{A}_{\mathcal{V}}, \mathcal{R}_{\mathcal{V}}, \mathcal{C}, \mathcal{L}_{\mathcal{M}}, \mathcal{D}_{v}\right)$ be the $V-M A F$ of a $V A F \Delta=(\mathcal{A}, \mathcal{R}, V$,val, $P)$. Then $E$ is a preferred extension of $(\mathcal{A}, \mathcal{R}, V, v a l, a)$, where a $\in P$, iff $E^{\prime} \cup$ $\left\{\left(x_{v} P y_{v^{\prime}}\right) \mid v>_{a} v^{\prime}\right\} \cup\left\{\left(>_{a}\right)\right\}$ is a preferred extension of $\Delta_{V}$, where:

1. $(x, y) \in \overrightarrow{E+}$ iff $(x \operatorname{def} y) \in E^{\prime}$

2. $y \in E+\operatorname{iff}(r-y) \in E^{\prime}$

3. $x \in E$ iff $(j-x) \in E^{\prime}$

Proof Given Lemma 6 it suffices to show that:

$E^{*}=E^{\prime} \cup\left\{\left(x_{v} P y_{v^{\prime}}\right) \mid v>_{a} v^{\prime}\right\} \cup\left\{\left(>_{a}\right)\right\}$ is a preferred extension of the metalevel formulation $\Delta_{a}=\left(\mathcal{A}_{a}, \mathcal{R}_{a}, \mathcal{C}, \mathcal{L}_{\mathcal{M}}, \mathcal{D}_{v}\right)$ of $(\mathcal{A}, \mathcal{R}, V, v a l, a)$, where a $\in P$, iff $E^{*}$ is a preferred extension of $\Delta_{V}$.

Firstly, note that it is straightforward to show that for each $\Delta_{a}$ :

1. $\mathcal{A}_{\mathrm{a}} \subseteq \mathcal{A}_{V}$, where $\mathcal{A}_{V}-\mathcal{A}_{\mathrm{a}}=\left\{\left(>_{\mathrm{a}^{\prime}}\right) \mid \mathrm{a}^{\prime} \neq \mathrm{a}, \mathrm{a}^{\prime} \in P\right\}$

2. $\mathcal{R}_{\mathrm{a}} \subseteq \mathcal{R}_{V}$, where $\mathcal{R}_{V}-\mathcal{R}_{\mathrm{a}}=$ $\left\{\left(\left(>_{a^{\prime}}\right),\left(x_{v} P y_{v^{\prime}}\right)\right),\left(\left(>_{a^{\prime}}\right),\left(>_{a}\right)\right),\left(\left(>_{a}\right),\left(>_{a^{\prime}}\right)\right) \mid a^{\prime} \neq a, a^{\prime} \in P, v>_{a} v^{\prime}\right\}$

a) Let $E^{*}$ be an admissible extension of some $\Delta_{a}$. We show that $E^{*}$ is an admissible extension of $\Delta_{V}$. Suppose $(\beta, \alpha) \in \mathcal{R}_{V}, \alpha \in E^{*}$, and:

$-(\beta, \alpha) \in \mathcal{R}_{\mathrm{a}}$, in which case $\exists \gamma \in E^{*}, \gamma \mathcal{R}_{\mathrm{a}} \beta$, and by $2, \gamma \mathcal{R}_{V} \beta$;

$-(\beta, \alpha) \notin \mathcal{R}_{\mathrm{a}}$, in which case by 1 and $2, \beta$ must be some $\left(>_{a^{\prime}}\right)$, $a^{\prime} \neq a$, $\alpha$ is either some $\left(x_{v} P y_{v^{\prime}}\right)$ or $\left(>_{a}\right)$. But then $\left(>_{a}\right) \mathcal{R}_{V}\left(>_{a^{\prime}}\right)$ where $\left(>_{a}\right) \in E^{*}$.

b) Let $E^{*}$ be an admissible extension of $\Delta_{V}$. We show that $E^{*}$ is an admissible extension of $\Delta_{\mathrm{a}}$. Suppose $\beta \mathcal{R}_{\mathrm{a}} \alpha, \alpha \in E^{*}$. By 2$), \beta \mathcal{R}_{V} \alpha$, and by the admissibility of $E^{*}, \exists \gamma \in E^{*}, \gamma \mathcal{R}_{V} \beta$. Suppose $\neg\left(\gamma \mathcal{R}_{a} \beta\right)$. Since $\beta \in \mathcal{A}_{\mathrm{a}}$, then by 1 and 2 , it must be that $\gamma$ is some $\left(>_{a^{\prime}}\right)$ s.t. $a^{\prime} \neq a$, and $\beta$ is a value preference argument $\left(x_{v} P y_{v^{\prime}}\right)$. But this contradicts $E^{*}$ is a conflict free subset of $\mathcal{A}_{V}$, given that $\left(>_{a}\right) \mathcal{R}_{V}\left(>_{a^{\prime}}\right)$ and $\left(>_{a}\right) \in E^{*}$

Suppose $E^{*}$ is a preferred extension of some $\Delta_{a}$. By a), $E^{*}$ is an admissible extension of $\Delta_{V}$. Suppose $E^{*}$ is not a preferred extension of $\Delta_{V}$. Then, $\exists E^{* *} \supset E^{*}$ s.t. $E^{* *}$ is an admissible extension of $\Delta_{V}$. Suppose $\alpha \in\left(E^{* *}-E^{*}\right)$, where $\alpha \in\left(\mathcal{A}_{\mathcal{V}}-\mathcal{A}_{\mathrm{a}}\right)$. But then this contradicts $E^{* *}$ is conflict free, given that $\alpha$ is either a value preference or audience argument, and $\left(>_{a}\right) \in E^{*},\left(>_{a}\right) \mathcal{R}_{V}\left(y_{v^{\prime}} P x_{v}\right)$ for every $\left(y_{v^{\prime}} P x_{v}\right) \notin E^{*}$ (see iii) in Lemma 6 ), and $\left(>_{a}\right) \mathcal{R}_{V}\left(>_{a^{\prime}}\right)$ for every $a^{\prime} \neq a$. Suppose $\alpha \in\left(E^{* *}-E^{*}\right)$, where $\alpha \in \mathcal{A}_{\mathrm{a}}$. By b), $E^{* *}$ is an admissible extension of $\Delta_{\mathrm{a}}$, contradicting $E^{*}$ is a preferred extension of $\Delta_{\mathrm{a}}$.

Suppose $E^{*}$ is a preferred extension of $\Delta_{V}$. By b), $E^{*}$ is an admissible extension of $\Delta_{a}$. Suppose $E^{*}$ is not a preferred extension of $\Delta_{a}$. Then, $\exists E^{* *} \supset E^{*}$ s.t. $E^{* *}$ is an admissible extension of $\Delta_{\mathrm{a}}$. But then by a), $E^{* *}$ is an admissible extension of $\Delta_{V}$, contradicting $E^{*}$ is a preferred extension of $\Delta_{V}$.

Theorem 6 Let $\Delta_{M}=\left(\mathcal{A}_{\mathcal{M}}, \mathcal{R}_{\mathcal{M}}, \mathcal{C}, \mathcal{L}_{\mathcal{M}}, \mathcal{D}_{v}\right)$ be the $V-M A F$ of a $V A F \Delta=$ $(\mathcal{A}, \mathcal{R}, V, v a l, P)$. Then for any $x \in \mathcal{A},(j-x) \in \mathcal{A}_{\mathcal{M}}$ :

1. $x$ is an objectively acceptable argument of $\Delta$ iff $(j-x)$ is a sceptically justified argument of $\Delta_{M}$ under the preferred semantics.

2. $x$ is a subjectively acceptable argument of $\Delta$ iff $(j-x)$ is a credulously justified argument of $\Delta_{M}$ under the preferred semantics. 


\section{Proof}

1) Left to right: Let $x$ be an objectively acceptable argument of $\Delta$. Suppose any preferred extension $E^{\prime}$ of $\Delta_{M}$. Since $E^{\prime}$ is complete, it must contain some set $\left\{\left(x_{v} P y_{v^{\prime}}\right)\right.$ $\left.\mid v>_{\mathrm{a}} v^{\prime}\right\} \cup\left\{\left(>_{\mathrm{a}}\right)\right\}$ where $\left(>_{\mathrm{a}}\right) \in \mathcal{A}_{\mathcal{M}}$. Suppose $(j-x) \notin E^{\prime}$. But then by the right to left half of Lemma 7, there is a corresponding preferred extension $E$ of some $(\mathcal{A}, \mathcal{R}, V, v a l, \mathrm{a})$ that does not contain $x$, contradicting $x$ is an objectively acceptable argument of $\Delta$.

Right to left: Let $E^{\prime}$ be any preferred extension of $\Delta_{M}$, where $E^{\prime}$ must contain some set $\left\{\left(x_{v} P y_{v^{\prime}}\right) \mid v>_{a} v^{\prime}\right\} \cup\left\{\left(>_{a}\right)\right\}$. We have $(j-x) \in E^{\prime}$, and by the right to left half of Lemma 7 , there is a corresponding preferred extension $E$ of some $(\mathcal{A}, \mathcal{R}, \mathrm{V}, \mathrm{val}, \mathrm{a})$ that contains $x$.

2) Left to right: Let $E$ be the preferred extension of some $(\mathcal{A}, \mathcal{R}, V, v a l, \mathrm{a}), x \in E$. By the left to right half of Lemma 7, there is a corresponding preferred extension $E^{\prime}$ of $\Delta_{M}$ s.t. $(j-x) \in E^{\prime}$.

Right to left: Let $E^{\prime}$ be any preferred extension of $\Delta_{M}$, where $E^{\prime}$ must contain some set $\left\{\left(x_{v} P y_{v^{\prime}}\right) \mid v>_{a} v^{\prime}\right\} \cup\left\{\left(>_{a}\right)\right\}$. Let $(j-x) \in E^{\prime}$. By the right to left half of Lemma 7 , there is a corresponding preferred extension $E$ of $(\mathcal{A}, \mathcal{R}, V, v a l, a)$ that contains $x$.

\subsection{Proofs for Section 3.6}

Lemmas 8, 9 and 10 are used in the proof of Theorem 7.

Lemma 8 Let $\Delta_{H}=\left(\left(\left(\mathcal{A}_{1}, \mathcal{R}_{1}\right), \mathcal{D}_{1}\right), \ldots,\left(\left(\mathcal{A}_{n}, \mathcal{R}_{n}\right), \mathcal{D}_{n}\right)\right)$ be the partition of the bounded hierarchical $\Delta=(\mathcal{A}, \mathcal{R}, \mathcal{D})$.

Let $\Delta_{M}=\left(\mathcal{A}_{\mathcal{M}}, \mathcal{R}_{\mathcal{M}}, \mathcal{C}, \mathcal{L}_{\mathcal{M}}, \mathcal{D}_{e}\right)$ be the metalevel formulation of $\Delta$ as defined in Definition 28.

Then there exists a partition $\Delta_{M H}$ of $\Delta_{M}$ such that:

$\Delta_{M H}=\left(\left(\left(\mathcal{A}_{1}^{\prime}, \mathcal{R}_{1}^{\prime}\right),\left(\mathcal{A}_{1-\mathcal{D}}^{\prime}, \mathcal{R}_{1-\mathcal{D}}^{\prime}\right)\right), \ldots,\left(\left(\mathcal{A}_{n}^{\prime}, \mathcal{R}_{n}^{\prime}\right),\left(\mathcal{A}_{n-\mathcal{D}}^{\prime}, \mathcal{R}_{n-\mathcal{D}}^{\prime}\right)\right)\right)$, where:

1. $\mathcal{A}_{\mathcal{M}}=\bigcup_{i=1}^{n}\left(\mathcal{A}_{i}^{\prime} \cup \mathcal{A}_{i-\mathcal{D}}^{\prime}\right)$ and $\mathcal{R}_{M}=\bigcup_{i=1}^{n}\left(\mathcal{R}_{i}^{\prime} \cup \mathcal{R}_{i-\mathcal{D}}^{\prime}\right)$

2. for $i=1 \ldots n,\left(\mathcal{A}_{i}^{\prime}, \mathcal{R}_{i}^{\prime}\right)$ are the arguments and attacks in the Dung $M A F$ formulation of $\left(\mathcal{A}_{i}, \mathcal{R}_{i}\right)$

3. for $i=1 \ldots n:(z,(y, x)) \in \mathcal{D}_{i}$ iff

$$
\begin{aligned}
& \left(\{(j-z),(r-z),(z D(y D x)),(y \operatorname{def} x)\} \subseteq \mathcal{A}_{i-\mathcal{D}}^{\prime},\right. \\
& \left\{((j-z),(r-z)),((r-z),(z D(y D x))),((z D(y D x)),(y \operatorname{def} x)) \subseteq \mathcal{R}_{i-\mathcal{D}}^{\prime}\right.
\end{aligned}
$$

4. $\left(\mathcal{A}_{n-\mathcal{D}}^{\prime}, \mathcal{R}_{n-\mathcal{D}}^{\prime}\right)=(\emptyset, \emptyset), \mathcal{D}_{n}=\emptyset$

Proof Proof is obvious. Intuitively, the partition $\Delta_{M H}$ corresponds to the partition $\Delta_{H}$, where each Dung framework $\left(\mathcal{A}_{i}, \mathcal{R}_{i}\right)$ is formulated as its Dung $M A F$ with arguments and attacks $\left(\mathcal{A}_{i}^{\prime}, \mathcal{R}_{i}^{\prime}\right)$, and the $\mathcal{D}_{i}$ attacks are formulated as the metalevel attacks in $\left(\mathcal{A}_{i-\mathcal{D}}^{\prime}, \mathcal{R}_{i-\mathcal{D}}^{\prime}\right)$. We illustrate with the example in Figure 17.

Lemma 9 Let $\Delta_{H}, \Delta_{M}$ and its partition $\Delta_{M H}=\left(\left(\left(\mathcal{A}_{1}^{\prime}, \mathcal{R}_{1}^{\prime}\right),\left(\mathcal{A}_{1-\mathcal{D}}^{\prime}, \mathcal{R}_{1-\mathcal{D}}^{\prime}\right)\right), \ldots\right.$, $\left.\left(\left(\mathcal{A}_{n}^{\prime}, \mathcal{R}_{n}^{\prime}\right),\left(\mathcal{A}_{n-\mathcal{D}}^{\prime}, \mathcal{R}_{n-\mathcal{D}}^{\prime}\right)\right)\right)$ be defined as in Lemma 8. Let us define the tuple:

$$
\left(\left(\left(\mathcal{A}_{1}^{\prime}, \mathcal{R}_{1}^{\prime}\right), \mathcal{R}_{1-\mathcal{D} r}^{\prime}\right), \ldots,\left(\left(\mathcal{A}_{n}^{\prime}, \mathcal{R}_{n}^{\prime}\right), \mathcal{R}_{n-\mathcal{D} r}^{\prime}\right)\right)
$$

where for $i=1 \ldots n-1$, the Dung framework $\left.\left(\mathcal{A}_{i-\mathcal{D}}^{\prime}, \mathcal{R}_{i-\mathcal{D}}^{\prime}\right)\right)$ with attacks $((j-$ $z),(r-z)),((r-z),(z D(y D x))),((z D(y D x)),(y \operatorname{def} x))$, is replaced by the singleton 
set of attacks $\mathcal{R}_{i-\mathcal{D} r}^{\prime}=\{((j-z),(y \operatorname{def} x))\}$ (notice that for $i=1 \ldots n-1,(y \operatorname{def} x)$ $\in \mathcal{A}_{i}^{\prime}$ and $\left.(j-z) \in \mathcal{A}_{i+1}^{\prime}\right)$

Let the reduction $\Delta_{M H r}$ of $\Delta_{M H}$ be defined as $\left(\mathcal{A}_{M H r}, \mathcal{R}_{M H r}\right)$ where ${ }^{12}$ :

$$
\mathcal{A}_{M H r}=\bigcup_{i=1}^{n} \mathcal{A}_{i}^{\prime} \text { and } \mathcal{R}_{M H r}=\bigcup_{i=1}^{n}\left(\mathcal{R}_{i}^{\prime} \cup \mathcal{R}_{i-\mathcal{D} r}^{\prime}\right) \text {. }
$$

Then $\forall \alpha \in \mathcal{A}_{M H r} \cap \mathcal{A}_{\mathcal{M}}$, for $s \in$ \{complete, grounded, preferred, stable $\}, \alpha$ is a credulously, respectively sceptically justified argument of $\Delta_{M H r}$ under the $s$ semantics, iff $\alpha$ is a credulously, respectively sceptically justified argument of $\Delta_{M}$ under the $s$ semantics.

Proof: $\Delta_{M H}$ is an expansion of $\Delta_{M H r}$ such that each $((j-z),(y \operatorname{def} x)) \in \mathcal{R}_{M H r}$ is expanded to obtain the set $\{((j-z),(r-z)),((r-z),(z D(y D x))),((z D(y D x)),(y \operatorname{def} x))\}$ in $\mathcal{R}_{M H r}$. The result therefore follows from Corollary 1.



Figure 17: $\Delta_{H}$ is the hierarchical partition of the $E A F \Delta$, and $\Delta_{M H}$ is the hierarchical partition of $\Delta$ 's metalevel formulation $\Delta_{M} . \Delta_{M H r}$ is the reduction of $\Delta_{M H}$.

${ }^{12}$ See Figure 17 for an example of $\Delta_{M H}$ and its reduction $\Delta_{M H r}$ 
Lemma 10 Let $E$ be an admissible extension of a bounded hierarchical $E A F \Delta=$ $(\mathcal{A}, \mathcal{R}, \mathcal{D})$. Let $x \in E, x \rightarrow^{E} y$ and $R s$ a reinstatement set for $x \rightarrow^{E} y$. Then $\forall F \supset E$ s.t. $F$ is admissible, $x \rightarrow^{F} y$ and $R s$ is a reinstatement set for $x \rightarrow^{F} y$.

Proof Given the partition $\left(\left(\left(\mathcal{A}_{1}, \mathcal{R}_{1}\right), \mathcal{D}_{1}\right), \ldots,\left(\left(\mathcal{A}_{n}, \mathcal{R}_{n}\right), \mathcal{D}_{n}\right)\right)$ of $\Delta$, we can partition $E$ into $E_{1} \cup \cdots \cup E_{n}$ where $x \in E_{i}$ iff $x \in \mathcal{A}_{i}$ and $(y, x) \in \mathcal{R}$ implies $(y, x) \in \mathcal{R}_{i}$, $(x, y) \in \mathcal{R}$ implies $(x, y) \in \mathcal{R}_{i}$, and $\left(y^{\prime},(x, y)\right) \in \mathcal{D}$ implies $\left(y^{\prime},(x, y)\right) \in \mathcal{D}_{i}$, $y^{\prime} \in \mathcal{A}_{i+1}$. We now prove the result by induction on $i$ :

Base case: Suppose $x \in E_{n-1}, x \rightarrow^{E} y$, and $y_{1}^{\prime}, \ldots, y_{m}^{\prime}$ s.t. for $k=1 \ldots m$, $\left(y_{k}^{\prime},(x, y)\right) \in \mathcal{D}_{n-1}$. Since $\mathcal{D}_{n}=\emptyset$, then the reinstatement set $R s$ for $x \rightarrow^{E} y$ is of the form $\left\{x \rightarrow^{E} y, x_{1}^{\prime} \rightarrow^{E} y_{1}^{\prime}, \ldots, x_{m}^{\prime} \rightarrow^{E} y_{m}^{\prime}\right\}$, since for $k=1 \ldots m \neg \exists\left(z,\left(x_{k}^{\prime}, y_{k}^{\prime}\right)\right) \in$ $\mathcal{D}$. The latter also implies that for any admissible $F$ s.t. $F \supset E, y_{k}^{\prime} \notin F$ (since otherwise $F$ would not be conflict free), $x \rightarrow^{F} y$ and $R s=\left\{x \rightarrow F y, x_{1}^{\prime} \rightarrow^{F}\right.$ $\left.y_{1}^{\prime}, \ldots, x_{m}^{\prime} \rightarrow^{F} y_{m}^{\prime}\right\}$ is a reinstatement set for $x \rightarrow^{F} y$.

Inductive hypothesis: The result holds for $x \in E_{j}, j>i$.

General case: Suppose $x \in E_{i}, x \rightarrow^{E} y$ and a reinstatement set $R s$ for $x \rightarrow^{E} y$. Suppose $\left\{y_{1}^{\prime}, \ldots, y_{m}^{\prime}\right\}=\left\{y^{\prime} \mid\left(y^{\prime},(x, y)\right) \in \mathcal{D}\right\}$. By assumption of $R s$, for $k=1 \ldots m$, $\exists x_{k}^{\prime} \in E_{i+1}$ s.t. $x_{k}^{\prime} \rightarrow^{E} y_{k}^{\prime}$. Hence, $R s=\left\{x \rightarrow^{E} y\right\} \cup \bigcup_{k=1}^{m} R s_{k}$ where $R s_{k}$ is a reinstatement set for $x_{k}^{\prime} \rightarrow^{E} y_{k}^{\prime}$. By inductive hypothesis, for $\mathrm{k}=1 \ldots m, x_{k}^{\prime} \rightarrow^{F} y_{k}^{\prime}$ and $R s_{k}$ is a reinstatement set for $x_{k}^{\prime} \rightarrow^{F} y_{k}^{\prime}$. Hence, $x \rightarrow^{F} y$ (since for $k=1 \ldots m$, $y_{k}^{\prime} \notin F$, given that by Proposition 2 in [38] no two arguments defeat ${ }_{F}$ each other in a conflict free $F$ ) and $\bigcup_{k=1}^{m} R s_{k} \cup\left\{x \rightarrow^{F} y\right\}$ is a reinstatement set for $x \rightarrow^{F} y$.

Theorem 7 Let $\Delta_{M}=\left(\mathcal{A}_{\mathcal{M}}, \mathcal{R}_{\mathcal{M}}, \mathcal{C}, \mathcal{L}_{\mathcal{M}}, \mathcal{D}_{e}\right)$ be the $E-M A F$ of a bounded hierarchical $E A F \Delta=(\mathcal{A}, \mathcal{R}, \mathcal{D})$. Then for $s \in\{$ complete, grounded, preferred, stable $\}$, $(j-x) \in \mathcal{A}_{\mathcal{M}}$ is a credulously, respectively sceptically, justified argument of $\Delta_{M}$ under the $s$ semantics, iff $x \in \mathcal{A}$ is a credulously, respectively sceptically, justified argument of $\Delta$ under the $s$ semantics.

Proof Let $\Delta_{H}=\left(\left(\left(\mathcal{A}_{1}, \mathcal{R}_{1}\right), \mathcal{D}_{1}\right), \ldots,\left(\left(\mathcal{A}_{n}, \mathcal{R}_{n}\right), \mathcal{D}_{n}\right)\right)$ be the partition of $\Delta$. Let $\Delta_{M H}$ be the partition of $\Delta_{M}$. Let $\Delta_{M H r}=\left(\mathcal{A}_{M H r}, \mathcal{R}_{M H r}\right)$ be the reduction of $\Delta_{M H}$, as defined by Lemma 9 on the basis of

$$
\left(\left(\left(\mathcal{A}_{1}^{\prime}, \mathcal{R}_{1}^{\prime}\right), \mathcal{R}_{1-\mathcal{D} r}^{\prime}\right), \ldots,\left(\left(\mathcal{A}_{n}^{\prime}, \mathcal{R}_{n}^{\prime}\right), \mathcal{R}_{n-\mathcal{D} r}^{\prime}\right)\right)
$$

Given Lemmas 8 and 9 , it suffices to show that:

$(j-x) \in \mathcal{A}_{M H r}$ is a credulously, respectively sceptically, justified argument of $\Delta_{M H r}$ under the $s$ semantics, iff $x \in \mathcal{A}$ is a credulously, respectively sceptically, justified argument of $\Delta$ under the $s$ semantics.

To show the above we show that: $E$ is an $s$ extension of $\Delta$ iff $E^{\prime}$ is an $s$ extension of $\Delta_{M H r}$, where:

1. $x \in E, x$ defeats $_{E} y$ and there is a reinstatement set for the defeat $x \rightarrow_{E} y$ iff $(x \operatorname{def} y),(r-y) \in E^{\prime}$

2. $x \in E$ iff $(j-x) \in E^{\prime}$

Observe that:

O1 Referring to the EAF $\Delta$ and its hierarchical partition $\Delta_{H}: \forall(\beta, \alpha) \in \mathcal{R}, \forall(\gamma,(\beta, \alpha)) \in$ $\mathcal{D},(\beta, \alpha) \in \mathcal{R}_{i}$ iff $(\gamma,(\beta, \alpha)) \in \mathcal{D}_{i}, \gamma \in \mathcal{A}_{i+1}$ 
O2 For $i=1 \ldots n$ : $\left(\mathcal{A}_{i}^{\prime}, \mathcal{R}_{i}^{\prime}\right)$ are the arguments and attacks in the Dung $M A F$ formulation of $\left(\mathcal{A}_{i}, \mathcal{R}_{i}\right)$ in the partition $\Delta_{H}$ of $\Delta$

O3 For $i=1 \ldots n-1,(\gamma, \delta) \in \mathcal{R}_{i-\mathcal{D} r}^{\prime}$ implies $\gamma \in \mathcal{A}_{i+1}^{\prime}, \delta \in \mathcal{A}_{i}^{\prime}$, and $\gamma$ is an argument of the form $(j-z), \delta$ is an argument of the form $(y \operatorname{def} x)$.

O4 For $i=1 \ldots n,((j-z),(y \operatorname{def} x)) \in \mathcal{R}_{i-\mathcal{D} r}^{\prime}$ iff $(z,(y, x)) \in \mathcal{D}_{i}$.

$O 1-O 4$ imply that $E$ can be partitioned into $E_{1}, \ldots, E_{n}$, and $E^{\prime}$ into $E_{1}^{\prime}, \ldots, E_{n}^{\prime}$, and that the theorem is shown by proving by induction on $i$, the following result:

$E^{i}=\left(E_{i} \cup \ldots \cup E_{n}\right)$ is an $s$ extension of $\left(\mathcal{A}^{i}, \mathcal{R}^{i}, \mathcal{D}^{i}\right)=\left(\mathcal{A}_{i} \cup \cdots \cup \mathcal{A}_{n}, \mathcal{R}_{i} \cup\right.$ $\left.\cdots \cup \mathcal{R}_{n}, \mathcal{D}_{i} \cup \cdots \cup \mathcal{D}_{n}\right)$ iff $E^{i^{\prime}}=\left(E_{i}^{\prime} \cup \ldots \cup E_{n}^{\prime}\right)$ is an $s$ extension of $\left(\mathcal{A}^{i^{\prime}}, \mathcal{R}^{i^{\prime}}\right)=$ $\left(\mathcal{A}_{i}^{\prime} \cup \cdots \cup \mathcal{A}_{n}^{\prime}, \mathcal{R}_{i}^{\prime} \cup \mathcal{R}_{i-\mathcal{D} r}^{\prime} \cup \cdots \cup \mathcal{R}_{n}^{\prime} \cup \mathcal{R}_{n-\mathcal{D} r}^{\prime}\right)$, where:

1. $z \in E^{i}, z$ defeats $_{E^{i}} y$ and there is a reinstatement set for the defeat $z \rightarrow_{E^{i}} y$ iff $(z \operatorname{def} y),(r-y) \in E^{i^{\prime}}$

2. $x \in E^{i}$ iff $(j-x) \in E^{i^{\prime}}$

1) $s=$ admissible. Firstly, note that:

$\mathrm{R} 1 E^{i}=\left(E_{i} \cup \ldots \cup E_{n}\right)$ is an admissible extension of $\left(\mathcal{A}^{i}, \mathcal{R}^{i}, \mathcal{D}^{i}\right)$ implies $\forall j>i$, $E^{j}=\left(E_{j} \cup \ldots \cup E_{n}\right)$ is an admissible extension of $\left(\mathcal{A}^{j}, \mathcal{R}^{j}, \mathcal{D}^{j}\right)$.

To show the above, assume $\alpha \in E^{j}, \beta \rightarrow E^{j} \alpha$, where given the partition of $\Delta$, if $(\gamma,(\beta, \alpha)) \in \mathcal{D}$ then $(\gamma,(\beta, \alpha)) \in \mathcal{D}^{j}, \gamma \in \mathcal{A}_{j+1}$. Hence $\beta \rightarrow{ }^{E^{i}} \alpha$, and by the admissibility of $E^{i}, \exists \gamma \in E^{i}, \gamma \rightarrow^{E^{i}} \beta$ and there is a reinstatement set $R S_{i}$ for $\gamma \rightarrow{ }^{E^{i}} \beta$. Given the partition of $\Delta, \gamma \in \mathcal{A}^{j}$ and if $(\delta,(\gamma, \beta)) \in \mathcal{D}$ then $(\delta,(\gamma, \beta)) \in \mathcal{D}^{j}, \delta \in \mathcal{A}_{j+1}$. Hence, it is straightforward to show that $\gamma \rightarrow^{E^{j}} \beta$ and there is a reinstatement set $R S_{j}$ for $\gamma \rightarrow^{E^{j}} \beta$.

$\mathrm{R} 2 E^{i^{\prime}}$ is an admissible extension of $\left(\mathcal{A}^{i^{\prime}}, \mathcal{R}^{i^{\prime}}\right)$ implies $\forall j>i, E^{j^{\prime}}$ is an admissible extension of $\left(\mathcal{A}^{j^{\prime}}, \mathcal{R}^{j^{\prime}}\right)$.

This follows given $\Delta$ 's partition, which implies that $\forall j>i$, no argument in $\left(\mathcal{A}^{i^{\prime}}-\mathcal{A}^{j^{\prime}}\right) \mathcal{R}^{i}$ attacks an argument in $\mathcal{A}^{j^{\prime}}$, and so for any $\alpha \in E^{j^{\prime}},(\beta, \alpha) \in \mathcal{R}^{i^{\prime}}$ iff $(\beta, \alpha) \in \mathcal{R}^{j^{\prime}}$, and $\exists \gamma \in E^{i^{\prime}}$ s.t. $(\gamma, \beta) \in \mathcal{R}^{i^{\prime}}$ iff $\gamma \in E^{j^{\prime}}$ and $(\gamma, \beta) \in \mathcal{R}^{j^{\prime}}$.

Base case $(i=n)$ : Since $\mathcal{D}_{n}=\emptyset, z$ defeats $_{E^{n}} y$ iff $z \mathcal{R}_{n} y$, and trivially there is a reinstatement set for $z \rightarrow{ }^{E^{n}} y$. Also, $\mathcal{R}_{n-\mathcal{D} r}=\emptyset$, and $\left(\mathcal{A}_{n}^{\prime}, \mathcal{R}_{n}^{\prime}\right)$ are the arguments and attacks in the Dung $M A F$ formulation of $\left(\mathcal{A}_{n}, \mathcal{R}_{n}\right)$. Hence, for $i=n$, the result follows immediately from Corollary 2.

Inductive hypothesis (IH): The result holds for $j>i$.

General Case:

Left to right half: Let $E^{i}=\left(E_{i} \cup \ldots \cup E_{n}\right)$ be an admissible extension of $\left(\mathcal{A}^{i}, \mathcal{R}^{i}, \mathcal{D}^{i}\right)$. We show that $E^{i^{\prime}}$ as defined above is an admissible extension of $\left(\mathcal{A}^{i^{\prime}}, \mathcal{R}^{i^{\prime}}\right)$. By R1, $E^{i+1}=\left(E_{i+1} \cup \ldots \cup E_{n}\right)$ is an admissible extension of $\left(\mathcal{A}^{i+1}, \mathcal{R}^{i+1}, \mathcal{D}^{i+1}\right)$, and by $I H, E^{i+1^{\prime}}=\left(E_{i+1}^{\prime} \cup \ldots \cup E_{n}^{\prime}\right)$ is an admissible extension of $\left(\mathcal{A}^{i+1^{\prime}}, \mathcal{R}^{i+1^{\prime}}\right)$. We show that $E^{i^{\prime}}$, where $E^{i^{\prime}} \supset E^{i+1^{\prime}}$, is an admissible extension of $\left(\mathcal{A}^{i^{\prime}} \mathcal{R}^{i^{\prime}}\right)$.

Suppose $x \in E_{i}$. Then $(j-x) \in E_{i^{\prime}}$. We show $(j-x)$ is acceptable w.r.t. $E^{i^{\prime}}$ : 
By definition of $\Delta$ and $\Delta_{M H r}, \exists(y, x) \in \mathcal{R}^{i}$ iff $\exists((y \operatorname{def} x),(j-x)) \in \mathcal{R}^{i^{\prime}}$. Suppose $y \mathcal{R}^{i} x$. By assumption of $x$ acceptable w.r.t. $E^{i}, \exists z \in E_{i}$ s.t. $z \rightarrow^{E^{i}} y$ and there is a reinstatement set for $z \rightarrow^{E^{i}} y$. By definition of $E^{i^{\prime}},(j-z),(z \operatorname{def} y),(r-y) \in E^{i^{\prime}}$, where $(r-y) \mathcal{R}^{i^{\prime}}(y \operatorname{def} x)$, and so $(j-x)$ is acceptable w.r.t. $E^{i^{\prime}}$.

The result is shown in full by showing that $(r-y)$ and $(z \operatorname{def} y)$ are acceptable w.r.t. $E^{i^{\prime}}$ :

Firstly, $(z \operatorname{def} y) \in E_{i}^{\prime}$ reinstates $(r-y)$ against the attack $(j-y) \mathcal{R}^{i^{\prime}}(r-y)$. Secondly, $(j-z) \in E_{i}^{\prime}$ reinstates $(z \operatorname{def} y)$ against the attack $(r-z) \mathcal{R}^{i^{\prime}}(z \operatorname{def} y)$. However, suppose $\exists\left(j-y^{\prime}\right) \in E_{i+1}^{\prime}$ s.t. $\left(\left(j-y^{\prime}\right),(z \operatorname{def} y)\right) \in \mathcal{R}_{i-\mathcal{D} r}^{\prime}$. Hence, $\left(y^{\prime},(z, y)\right) \in \mathcal{D}_{i}$, and by assumption of a reinstatement set for $z \rightarrow{ }^{E^{i}} y, \exists z^{\prime} \in E_{i+1}, z^{\prime} \rightarrow^{E^{i}} y^{\prime}$, and there is a reinstatement set for $z^{\prime} \rightarrow^{E^{i}} y^{\prime}$. By R1 and $I H$ above, $\left(j-z^{\prime}\right),\left(z^{\prime} \operatorname{def} y^{\prime}\right) \in E_{i+1}^{\prime}$, and given $\left(z^{\prime} \operatorname{def} y^{\prime}\right) \mathcal{R}^{i+1^{\prime}}\left(j-y^{\prime}\right)$ and so $\left(z^{\prime} \operatorname{def} y^{\prime}\right) \mathcal{R}^{i^{\prime}}\left(j-y^{\prime}\right),(z \operatorname{def} y)$ is acceptable w.r.t. $E^{i^{\prime}}$.

Right to left half: Let $E^{i^{\prime}}=\left(E_{i}^{\prime} \cup \ldots \cup E_{n}^{\prime}\right)$ be an admissible extension of $\left(\mathcal{A}^{i^{\prime}}, \mathcal{R}^{i^{\prime}}\right)$. We show that $E^{i}$ as defined above is an admissible extension of $\left(\mathcal{A}^{i}, \mathcal{R}^{i}, \mathcal{D}^{i}\right)$. By R2, $E^{i+1^{\prime}}=\left(E_{i+1}^{\prime} \cup \ldots \cup E_{n}^{\prime}\right)$ is an admissible extension of $\left(\mathcal{A}^{i+1^{\prime}}, \mathcal{R}^{i+1^{\prime}}\right)$, and by $I H$, $E^{i+1}=\left(E_{i+1} \cup \ldots \cup E_{n}\right)$ is an admissible extension of $\left(\mathcal{A}^{i+1}, \mathcal{R}^{i+1}, \mathcal{D}^{i+1}\right)$. We show that $E^{i}$, where $E^{i} \supset E^{i+1}$, is an admissible extension of $\left(\mathcal{A}^{i}, \mathcal{R}^{i}, \mathcal{D}^{i}\right)$.

Suppose $(j-x) \in E_{i}^{\prime}$. Then $x \in E_{i}$. We show $x$ is acceptable w.r.t. $E^{i}$ :

By definition of $\Delta$ and $\Delta_{M H r}, \exists(y, x) \in \mathcal{R}^{i}$ iff $\exists((y \operatorname{def} x),(j-x)) \in \mathcal{R}^{i^{\prime}}$. Suppose $((y \operatorname{def} x),(j-x)) \in \mathcal{R}^{i^{\prime}}$. Since $E^{i^{\prime}}$ is admissible, either:

a) $\exists\left(j-x^{\prime}\right) \in E_{i+1}^{\prime}$ s.t. $\left(j-x^{\prime}\right) \mathcal{R}_{i-\mathcal{D} r}^{\prime}(y \operatorname{def} x)$, and so $\left(x^{\prime},(y, x)\right) \in \mathcal{D}_{i}$. By R2 and $I H, x^{\prime} \in E_{i+1}$, and so $y \nrightarrow^{E^{i}} x$,

or;

b) $\exists(r-y) \in E_{i}^{\prime}$ s.t. $(r-y) \mathcal{R}^{i^{\prime}}(y \operatorname{def} x)$, and since $(j-y) \mathcal{R}^{i^{\prime}}(r-y), \exists(z \operatorname{def} y) \in E_{i}^{\prime}$ s.t. $(z \operatorname{def} y) \mathcal{R}^{i^{\prime}}(j-y)$ (and so $\left.z \mathcal{R}^{i} y\right)$ and since $(r-z) \mathcal{R}^{i^{\prime}}(z \operatorname{def} y), \exists(j-z) \in E_{i}^{\prime}$ s.t. $(j-z) \mathcal{R}^{i^{\prime}}(z \operatorname{def} y)$. By definition, $z \in E_{i}$.

Suppose $\left(j-y_{1}^{\prime}\right) \ldots\left(j-y_{m}^{\prime}\right) \in \mathcal{A}_{i+1}^{\prime}$ s.t. for $k=1 \ldots m,\left(j-y_{k}^{\prime}\right) \mathcal{R}^{i^{\prime}}(z \operatorname{def} y)$, in which case for $k=1 \ldots m,\left(y_{k}^{\prime},(z, y)\right) \in \mathcal{D} i$. For each such $\left(j-y_{k}^{\prime}\right)$, by admissibility of $E^{i^{\prime}}, \exists\left(z^{\prime} \operatorname{def} y_{k}^{\prime}\right) \in E_{i+1}^{\prime}$ s.t. $\left(z^{\prime} \operatorname{def} y_{k}^{\prime}\right) \mathcal{R}^{i^{\prime}}\left(j-y_{k}^{\prime}\right)$, and since $\left(r-z^{\prime}\right) \mathcal{R}^{i^{\prime}}\left(z^{\prime} \operatorname{def} y_{k}^{\prime}\right)$, $\exists\left(j-z^{\prime}\right) \in E_{i+1}^{\prime}$ s.t. $\left(j-z^{\prime}\right) \mathcal{R}^{i^{\prime}}\left(r-z^{\prime}\right)$. By R2 and $I H, z^{\prime} \in E_{i+1}, z^{\prime} \rightarrow{ }^{E^{i+1}} y_{k}^{\prime}$, and there is a reinstatement set $R s_{k}$ for $z^{\prime} \rightarrow E^{E^{i+1}} y_{k}^{\prime}$.

Hence, we have $z \in E_{i}, z \rightarrow^{E^{i}} y$, and there is a reinstatement set $\bigcup_{k=1}^{m} R s_{k} \cup\left\{z \rightarrow{ }^{E^{i}}\right.$ $y$ \} for $z \rightarrow^{E^{i}} y$. Hence $x$ is acceptable w.r.t. $E^{i}$.

We have shown: $\mathbf{1 . 1}=$ the left to right half for $s=$ admissible, and; $\mathbf{1 . 2}=$ the right to left half for $s=$ admissible. We define functions $f$ and $g$ s.t.

For any admissible extension $E$ of $\Delta, E^{\prime}=h(E)$.

For any admissible extension $E^{\prime}$ of $\Delta_{M H r}, E=g\left(E^{\prime}\right)$.

From hereon, we will let $\Delta^{\prime}=\left(\mathcal{A}^{\prime}, \mathcal{R}^{\prime}\right)$ denote $\Delta_{M H r}$. We show that:

a) $h$ is monotonically strictly increasing.

Suppose $E$ and by $\mathbf{1 . 1}$ the corresponding admissible $E^{\prime}=h(E)$. Suppose $E \subset F$ and by $\mathbf{1 . 1}$ the corresponding admissible $F^{\prime}=h(F)$. Suppose $\exists x \in E$ s.t. $x \rightarrow^{E} y$ 
and there is a reinstatement set for $x \rightarrow^{E} y$. By lemma 10, $x \rightarrow^{F} y$ and there is a reinstatement set for $x \rightarrow^{F} y$, and so it must be that $E^{\prime} \subset F^{\prime}$.

b) $g$ is monotonically strictly increasing.

Suppose $E^{\prime}$ and by $\mathbf{1 . 2}$ the corresponding admissible $E=g\left(E^{\prime}\right)$. Suppose $E^{\prime} \subset F^{\prime}$, where:

$\forall \alpha \in\left(F^{\prime}-E^{\prime}\right)$, if $\alpha$ is of the form $(j-x)$, or $\alpha$ is of the form $(r-y)$ or $(x \operatorname{def} y)$, then $x \notin E$, respectively $\neg \exists x \in E$ s.t. $x \rightarrow^{E} y$ and there is a reinstatement set for $x \rightarrow^{E} y$, since otherwise, by 1.1, we would have $(j-x) \in E^{\prime}$, respectively $(r-y)$ or $(x \operatorname{def} y) \in E^{\prime}$.

By 1.2, let $F$ be the corresponding admissible extension of $\Delta$. If $\exists(j-x) \in\left(F^{\prime}-E^{\prime}\right)$ then $x \in F$, and by i), $E \subset F$. If $\exists(r-y) \in\left(F^{\prime}-E^{\prime}\right)$ or $\exists(x \operatorname{def} y) \in\left(F^{\prime}-E^{\prime}\right)$, then $\exists x \in F$ s.t. $x \rightarrow^{F} y$ and there is a reinstatement set for $x \rightarrow^{F} y$. Given i), there are three cases to consider: 1) Suppose $x \in E$ and $x \nrightarrow^{E} y$. Then given $E \subseteq F$ it cannot be that $x \rightarrow^{F} y$; 2) Suppose $x \in E, x \rightarrow^{E} y$ and there is no reinstatement set for $x \rightarrow^{E} y$. But then since there is a reinstatement set for $x \rightarrow^{F} y$, it must be that $E \subset F$; 3) Suppose $x \notin E$. Then $E \subset F$.

Left to right and right to left half for $s \in\{$ complete, grounded, preferred $\}$. Given a) and b), the theorem is shown to hold in exactly the same way as in Lemma 3.

Left to right half for $s=$ stable: Suppose $E$ is stable. Hence $E$ is complete ${ }^{13}$. By the left to right for $s=$ complete, $E^{\prime}$ is complete. Suppose $\alpha \notin E^{\prime}, \alpha$ is not $\mathcal{R}^{\prime}$ attacked by an argument in $E^{\prime}$. There are three cases to consider:

a) $\alpha$ is some $(j-x) \notin E^{\prime}$. Then $x \notin E, \exists y \in E$ s.t. $y \rightarrow^{E} x$. Notice that there must be a reinstatement set for $y \rightarrow^{E} x$, since to suppose otherwise means that for some $y^{\prime} \in E, x^{\prime} \notin E$ s.t. $y^{\prime} \rightarrow^{E} x^{\prime}$, then $\exists\left(x^{\prime \prime},\left(y^{\prime}, x^{\prime}\right)\right) \in \mathcal{D}$ s.t. $x^{\prime \prime} \notin E$, and $\neg \exists y^{\prime \prime} \in E$ s.t. $y^{\prime \prime} \rightarrow^{E} x^{\prime \prime}$, contradicting $E$ is stable. Hence, $(y \operatorname{def} x) \in E^{\prime}$, where $(y \operatorname{def} x) \mathcal{R}^{\prime}(j-x)$. b) Suppose some $(r-x) \notin E^{\prime}$, and so given $(j-x) \mathcal{R}^{\prime}(r-x),(j-x) \notin E^{\prime}$. But then we have shown in a) that $(y \operatorname{def} x) \in E^{\prime}$, where $(y \operatorname{def} x) \mathcal{R}^{\prime}(j-x)$, and so $(r-x)$ is acceptable w.r.t. $E^{\prime}$, contradicting $E^{\prime}$ is complete.

c) Suppose some $(y \operatorname{def} x) \notin E^{\prime}$, and so given $(r-y) \mathcal{R}^{\prime}(y \operatorname{def} x),(r-y) \notin E^{\prime}$. But then we have shown in b) that $(j-y) \in E^{\prime},(j-y) \mathcal{R}^{\prime}(r-y)$. Suppose some $\left(j-x^{\prime}\right)$ s.t. $\left(j-x^{\prime}\right) \mathcal{R}^{\prime}(y \operatorname{def} x),\left(j-x^{\prime}\right) \notin E^{\prime}$. We have shown in a) that $\left(y^{\prime} \operatorname{def} x^{\prime}\right) \in E^{\prime}$, where $\left(y^{\prime} \operatorname{def} x^{\prime}\right) \mathcal{R}^{\prime}\left(j-x^{\prime}\right)$. Hence $(y \operatorname{def} x)$ is acceptable w.r.t. $E^{\prime}$, contradicting $E^{\prime}$ is complete.

Right to left halffor $s=$ stable: Suppose $E^{\prime}$ is a stable extension. By the right to left for $s=$ complete, $E$ is complete. Suppose some $x \notin E$. Then $(j-x) \notin E^{\prime}, \exists(y \operatorname{def} x) \in E^{\prime}$ s.t. $(y \operatorname{def} x) \mathcal{R}^{\prime}(j-x)$, and by the right to left half for $s=$ complete, $y \in E, y \rightarrow^{E} x$.

\section{References}

[1] L. Amgoud. A formal framework for handling conflicting desires. In Proc. 7th European Conference on Symbolic and Quantitative Approaches to Reasoning with Uncertainty (ECSQARU'2003), pages 552-563, 2003.

\footnotetext{
${ }^{13}$ Suppose otherwise. Then $x \notin E$ and $x$ acceptable w.r.t. $E$. Since $E$ is stable, $\exists y \in E$ s.t. $y \rightarrow{ }^{E} x$ and by acceptability of $x, \exists z \in E$ s.t. $z \rightarrow^{E} y$, contradicting Proposition 2 in [38] which states that no two arguments defeat $_{E}$ each other in a conflict free $E$.
} 
[2] L. Amgoud and C. Cayrol. A reasoning model based on the production of acceptable arguments. Annals of Mathematics and Artificial Intelligence, 34(1-3):197215, 2002.

[3] L. Amgoud, C. Cayrol, M. Lagasquie-Schiex, and P. Livet. On bipolarity in argumentation frameworks. International Journal of Intelligent Systems, 23(10):1062-1093, 2008.

[4] L. Amgoud, Y. Dimopolous, and P. Moraitis. A unified and general framework for argumentation-based negotiation. In Proc. 6th International Joint Conference on Autonomous Agents and Multi-Agents Systems (AAMAS'2007), pages 14 - 18, 2007.

[5] ArguGRID. www.argugrid.org. $2007-2009$.

[6] ASPIC. Argumentation services platform with integrated components (www.argumentation.org). 2004 - 2007.

[7] K. M. Atkinson, T. J. M. Bench-Capon, and P. McBurney. Computational representation of practical argument. Synthese, 152(2):157-206, September 2006.

[8] P. Baroni, F. Cerutti, M. Giacomin, and G. Guida. Encompassing attacks to attacks in abstract argumentation frameworks. In Proc. 10thth European Conference on Symbolic and Quantitative Approaches to Reasoning with Uncertainty, pages 83-94, 2009.

[9] P. Baroni and M. Giacomin. Resolution-based argumentation semantics. In Proc. 2nd International Conference on Computational Models of Argument, pages 2536, Toulouse, France, May, 2008. IOS Press.

[10] H. Barringer, D. M. Gabbay, and J. Woods. Temporal dynamics of support and attack networks: From argumentation to zoology. In Mechanizing Mathematical Reasoning, pages 59-98, 2005.

[11] T. J. M. Bench-capon. Agreeing to differ: modelling persuasive dialogue between parties with different values. Informal Logic, 22:2002, 2003.

[12] T. J. M. Bench-Capon. Persuasion in practical argument using value-based argumentation frameworks. Journal of Logic and Computation, 13(3):429-448, 2003.

[13] T. J. M. Bench-Capon, S. Doutre, and P. E. Dunne. Audiences in argumentation frameworks. Artificial Intelligence, 171(1):42-71, 2007.

[14] T. J. M. Bench-Capon and P. E. Dunne. Argumentation in artificial intelligence. Artificial Intelligence, 171:10-15, 2007.

[15] T. J. M. Bench-Capon and S. Modgil. Case law in extended argumentation frameworks. In ICAIL, pages 118-127, 2009.

[16] T.J.M. Bench-Capon. Representation of case law as an argumentation framework. In Proceedings of JURIX 2002, pages 103-112, The Netherlands, 2002. IOS Press. 
[17] P. Besnard and A. Hunter. Practical first-order argumentation. In Proc. 20th American National Conference on Artificial Intelligence (AAAI'2005), pages 590-595, 2005.

[18] A. Bochman. Collective argumentation and disjunctive programming. Journal of Logic and Computation, 13 (3):405-428, 2003.

[19] G. Boella, J. Hulstijn, and L. W. N. van der Torre. Argmas. In 2nd International Workshop on Argumentation in Multi-Agent Systems, pages 29-41. Springer, 2005.

[20] G. Boella, L.W.N van der Torre, and S. Villata. Social viewpoints for arguing about coalitions. In 11th Pacific Rim International Conference on Multi-agents, pages 66-77, 2008.

[21] A. Bondarenko, P.M. Dung, R.A. Kowalski, and F. Toni. An abstract, argumentation-theoretic approach to default reasoning. Artificial Intelligence, 93:63-101, 1997.

[22] M. Caminada. On the issue of reinstatement in argumentation. In 10th European Conference on Logic in Artificial Intelligence (JELIA), pages 111-123, 2006.

[23] M. Caminada. An algorithm for computing semi-stable semantics. In 9th European Conference on Symbolic and Quantitative Approaches to Reasoning with Uncertainty (ECSQARU'09), pages 222-234, 2007.

[24] C. Cayrol, S. Doutre, and J. Mengin. On Decision Problems related to the preferred semantics for argumentation frameworks. Journal of Logic and Computation, 13(3):377-403, 2003.

[25] D.M.Berman and C.L. Hafner. Representing teleological structure in case-based legal reasoning: the missing link. In Proceedings of the Fourth International Conference on Artificial Intelligence and Law, pages 50-59, New York, 2003. ACM Press.

[26] P. M. Dung. An argumentation semantics for logic programming with explicit negation. In Proceedings of the Tenth Logic Programming Conference, pages 616-630, Cambridge, MA, 1993. MIT Press.

[27] P. M. Dung. On the acceptability of arguments and its fundamental role in nonmonotonic reasoning, logic programming and $n$-person games. Artificial Intelligence, $77: 321-357,1995$.

[28] P. E. Dunne and T. J. M. Bench-Capon. Two party immediate response dispute: Properties and efficiency. Artificial Intelligence, 149:221-250, 2003.

[29] P. E. Dunne, A. Hunter, P. McBurney, S. Parsons, and M. Wooldridge. Inconsistency tolerance in weighted argument systems. In 8th International Joint Conference on Autonomous Agents and Multiagent Systems (AAMAS 2009), pages 851-858, 2009.

[30] Estrella. www.estrellaproject.org. $2006-2008$. 
[31] G. Governatori and M. J. Maher. An argumentation-theoretic characterization of defeasible logic. In Proceedings of the Fourteenth European Conference on Artificial Intelligence, pages 469-473, 2000.

[32] D. Hitchcock, P. McBurney, and S. Parsons. A framework for deliberation dialogues. In Proc. 4th Biennial Conference of the Ontario Society for the Study of Argumentation, 2001

[33] S. Kaci and L.W.N. van der Torre. Preference-based argumentation: Arguments supporting multiple values. International Journal of Approximate Reasoning, 48(3):730-751, 2008.

[34] A. Kakas and P. Moraitis. Argumentation based decision making for autonomous agents. In Proc. Second international joint conference on autonomous agents and multiagent systems, pages 883-890, 2003.

[35] S. Modgil. Hierarchical argumentation. In Proc. 10th European Conference on Logics in Artificial Intelligence, pages 319-332, Liverpool, UK, 2006.

[36] S. Modgil. Value based argumentation in hierarchical argumentation frameworks. In Proc. 1st Int. Conference on Computational Models of Argument, pages 297308, Liverpool, UK, 2006.

[37] S. Modgil. An argumentation based semantics for agent reasoning. In Proc. Workshop on Languages, methodologies and development tools for multi-agent systems (LADS 07), pages 37-53, Durham, UK, 2007.

[38] S. Modgil. Reasoning about preferences in argumentation frameworks. Artificial Intelligence, 173(9-10):901-934, 2009.

[39] S. Modgil. Labellings and games for extended argumentation frameworks. In Twenty-first International Joint Conference on Artificial Intelligence (IJCAI-09), USA, July 11-15, 2009.

[40] S. Modgil. An abstract theory of argumentation that accommodates defeasible reasoning about preferences. In Proc. 9th European Conference on Symbolic and Quantitative Approaches to Reasoning with Uncertainty, pages 648-659, Tunisia, October 2007.

[41] S. Modgil and T. J. M. Bench-Capon. Integrating object and meta-level value based argumentation. In Proc. 2nd Int. Conf. on Computational Models of Argument, pages 240-251, 2008.

[42] S. Modgil and M. Caminada. Proof theories and algorithms for abstract argumentation frameworks. In I. Rahwan and G. Simari, editors, Argumentation in AI. Springer-Verlag, 2009.

[43] S. Modgil and M. Luck. Argumentation based resolution of conflicts between desires and normative goals. In Proc. 5th Int. Workshop on Argumentation in Multi-Agent Systems, pages 252-263, 2008.

[44] S. H. Nielsen and S. Parsons. A generalization of dung's abstract framework for argumentation: Arguing with sets of attacking arguments. In 3rd International Workshop on Argumentation in Multi-agent Systems (ArgMAS 2006), pages 5473, 2006. 
[45] N. Oren and T. J. Norman, editors. Semantics for Evidence-Based Argumentation, Toulouse, France, 2008.

[46] J. L. Pollock. Defeasible reasoning. Cognitive Science, 11:481-518, 1987.

[47] H. Prakken. Coherence and flexibility in dialogue games for argumentation. Journal of logic and computation, 15:1009-1040, 2005.

[48] H. Prakken and G. Sartor. Argument-based extended logic programming with defeasible priorities. Journal of Applied Non-Classical Logics, 7:25-75, 1997.

[49] R. Reiter. A logic for default reasoning. Artif. Intell., 13(1-2):81-132, 1980.

[50] J.R. Searle. Rationality in Action. MIT Press, Cambridge, MA, 2001.

[51] B. Verheij. A labeling approach to the computation of credulous acceptance in argumentation. In International Joint Conference on Aritificial Intelligence (IJCAI), pages 623-628, 2007.

[52] G. Vreeswijk. An algorithm to compute minimally grounded and admissible defence sets in argument systems. In Proc. 1st International Conference on Computational Models of Argument, pages 109-120, UK, 2006.

[53] G. A. W. Vreeswijk and H. Prakken. Credulous and sceptical argument games for preferred semantics. In Proc. 7th European Workshop on Logic for Artificial Intelligence, pages 239-253, 2000.

[54] D. N. Walton. Argument Schemes for Presumptive Reasoning. Lawrence Erlbaum Associates, Mahwah, NJ, USA, 1996.

[55] M. Wooldridge, P. McBurney, and S. Parsons. On the meta-logic of arguments. In Proc. Fourth international joint conference on Autonomous agents and multiagent systems (AAMAS'05), pages 560-567, NY, USA, 2005. ACM Press. 